University of Louisville

ThinkIR: The University of Louisville's Institutional Repository

Electronic Theses and Dissertations

8-2016

\title{
Type 2 diabetes mellitus (T2DM) onset and "remission".
}

Srikanth Tangelloju

Follow this and additional works at: https://ir.library.louisville.edu/etd

Part of the Public Health Commons

\section{Recommended Citation}

Tangelloju, Srikanth, "Type 2 diabetes mellitus (T2DM) onset and "remission"." (2016). Electronic Theses and Dissertations. Paper 2515.

https://doi.org/10.18297/etd/2515

This Doctoral Dissertation is brought to you for free and open access by ThinkIR: The University of Louisville's Institutional Repository. It has been accepted for inclusion in Electronic Theses and Dissertations by an authorized administrator of ThinkIR: The University of Louisville's Institutional Repository. This title appears here courtesy of the author, who has retained all other copyrights. For more information, please contact thinkir@louisville.edu. 


\title{
TYPE 2 DIABETES MELLITUS (T2DM) ONSET AND "REMISSION"
}

\author{
By
}

Srikanth Tangelloju

\begin{abstract}
A Dissertation
Submitted to the Faculty of the

School of Public Health and Information Sciences of the University of Louisville in Partial Fulfillment of the Requirements for the Degree of Doctor of Philosophy in Public Health Sciences
\end{abstract}

Department of Health Management and Systems Sciences University of Louisville Louisville, Kentucky

August 2016 
Copyright 2016 by Srikanth Tangelloju

All rights reserved 

TYPE 2 DIABETES MELLITUS (T2DM) ONSET AND "REMISSION"

$$
\text { By }
$$

Srikanth Tangelloju

A Dissertation Approved on

July 20, 2016

By the following Dissertation Committee

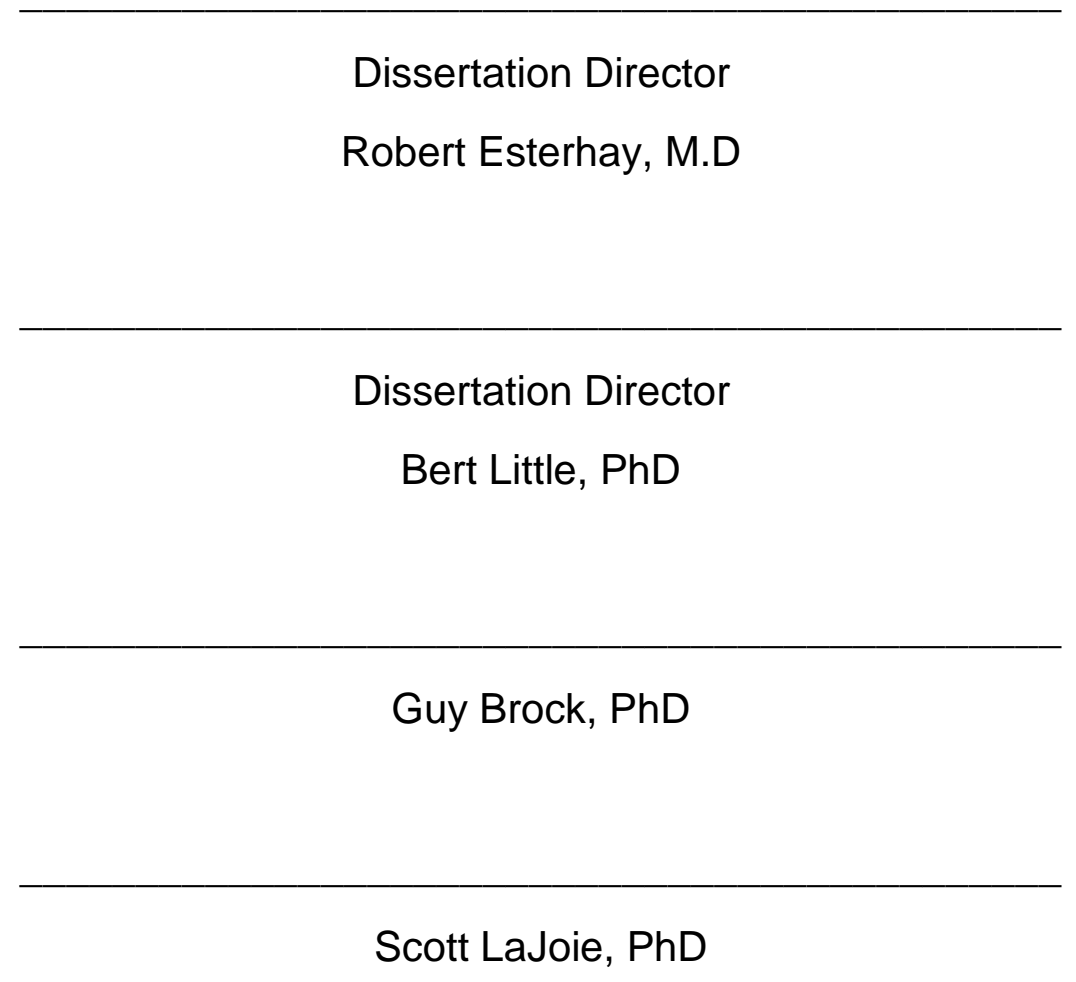




\section{DEDICATION}

This thesis is dedicated to my loving family who has always been my greatest support.

Grandparents, late Dr. T. Balabrahmam and Ratnamma

Father, Dr. T. Veeranarayana and mother, Dr. K. Ravindramani

My outrageously lovely wife, Swathi

My daughter, Srimayukha

My brother, Sridhar

My sister-in-law, Sumedha

My niece, Mihira

I love you all! 


\section{ACKNOWLEDGEMENTS}

I would like to thank my committee for their exceptional guidance, support, and constructive criticism throughout this study. I would like to especially thank Dr. Robert Esterhay and Dr. Bert Little for their mentorship. You both are incredibly awesome; I wish every teacher were like you.

I would like to thank Humana Inc. for allowing me to use their data for my Ph.D. dissertation. I would like to thank my manager Aamer Charania and colleagues Sari Hopson, Maresa Corder and Paul Morris for their guidance.

I would like to thank my family for their endless love, support and encouragement throughout my life; especially my soul mate Swathi. Her unconditional love and sacrifice allowed me to focus on my study. I am blessed to have her in my life. 


\title{
ABSTRACT
}

\section{TYPE 2 DIABETES MELLITUS (T2DM) ONSET AND "REMISSION"}

\author{
Srikanth Tangelloju \\ July 20, 2016
}

\section{Background}

Type 2 diabetes mellitus (T2DM) is a progressive disease condition. As the disease progresses the function of beta $(\beta)$ cells (cells which regulate insulin) decline, thereby increasing the circulating blood glucose levels. If left unmanaged the disease progresses from onset to development of T2DM related complications and eventually death. When managed, in certain individuals, T2DM onset can be prevented and/or slowed down and in certain T2DM individuals, remission is observed. There are some known modifiable risk factors such as diet and physical activities that are associated with the speed and direction of progression, but much is still unknown. In order to develop effective intervention and management programs, it is first important to understand the factors that predict speed and direction of T2DM progression. 


\section{Purpose}

This dissertation evaluated T2DM disease progression with three key objectives: a) evaluate factors that predict T2DM onset; b) evaluate rate of T2DM "remission" and, c) evaluate factors that predict T2DM "remission" in Medicare patients 65 years and older who did not undergo bariatric surgery.

\section{Methods}

A retrospective cohort analysis of a Medicare Advantage health plan was conducted using administrative data. An individual was identified as T2DM if they had: $\geq 2$ medical claims for T2DM coded 250.xx excluding type 1 diabetes; or $\geq 2$ pharmacy claims related to T2DM; or $\geq 2$ combined medical claims, pharmacy claims for T2DM in 12 months. A T2DM individual was in "remission" if they had no T2DM related claims for more than 12 months continuously. This is different from the standard American Diabetes Association definition of remission which includes $\mathrm{HbA} 1_{c}$ values and hence is represented in quotation (as "remission"). 89,390 individuals were evaluated for T2DM onset and 10,059 T2DM individuals were evaluated for T2DM "remission" over a period of 8 years from 2008 to 2015. Cox proportional hazards was used to identify significant variables associated with T2DM onset and "remission." 


\section{Results}

The factors that were significantly associated with T2DM onset were: male gender; non-white ethnicity (African American, Hispanics); statin use; hypertension; hyperlipidemia; heart failure; ulcer of lower limbs; atherosclerosis; other retinopathy; angina pectoris; blindness and low vision; absence of other chronic ischemic heart disease (IHD) $(p<0.05)$.

T2DM "remission" does occur without bariatric surgery. 4.97\% $(95 \% \mathrm{Cl}, 3.05 \%-8.00 \%)$ of Medicare Patients 65 years and older met the definition of T2DM "remission" in the study cohort.

The factors associated with T2DM "remission" were no statin use; low diabetes complications severity index score (DCSI); no hypertension; no hyperlipidemia; no neuropathy; no retinopathy $(p<0.05)$. Race (Asian/Hispanic). Other chronic ischemic heart disease (IHD) and female gender were significantly associated with T2DM "remission" $(p<0.05)$.

\section{Conclusions}

In line with previously published studies, the study presented here also found that hyperlipidemia, hypertension, gender and race are significantly associated with T2DM onset. In addition to these known factors, this study identified additional factors associated with T2DM onset such as: statin use; hypertension; hyperlipidemia; heart failure; ulcer of lower limbs; atherosclerosis; other retinopathy; angina pectoris; blindness and low vision and absence of other chronic ischemic heart disease. 
This study found that in Medicare T2DM patients 65 years and older "remission" does occur without bariatric surgery. This study verified known factors such as absence of dyslipidemia and race to be associated with T2DM "remission." In addition to these known factors this study found that no statin use; low diabetes complications severity index score (DCSI); no hypertension; no neuropathy; no retinopathy; presence of other chronic ischemic heart disease (IHD) and female gender were significantly associated with T2DM "remission."

These findings could be used for the development of T2DM related disease intervention and management programs. The DCSI score of T2DM individuals could be used to help stratify them based on T2DM severity for design and individualized, targeted outreach.

Lastly, in this study hypertension, hyperlipidemia, statins use were associated with T2DM onset and T2DM "remission." Since these factors are also associated with metabolic disease, their mutual relation needs to be evaluated further, upon availability of laboratory and physician notes data. Metabolic disease is a known predictor for T2DM onset and future studies might find that metabolic disease might also be a predictor for T2DM "remission." 


\section{TABLE OF CONTENTS}

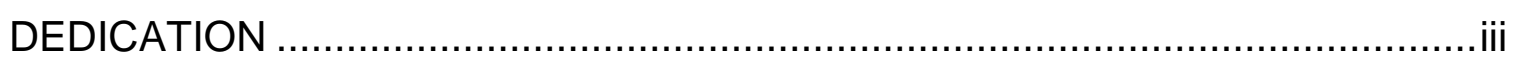

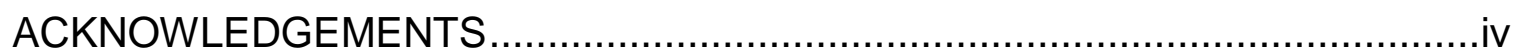

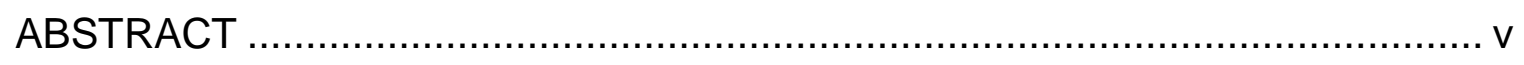

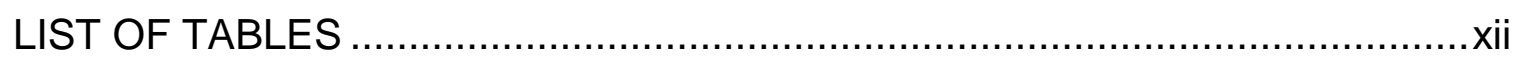

LIST OF FIGURES ..............................................................................

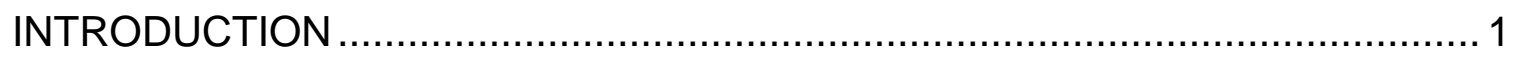

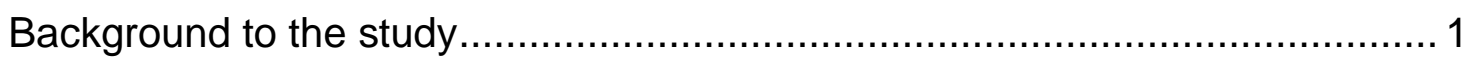

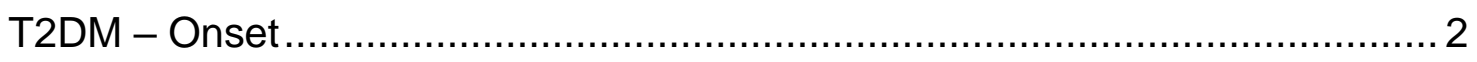

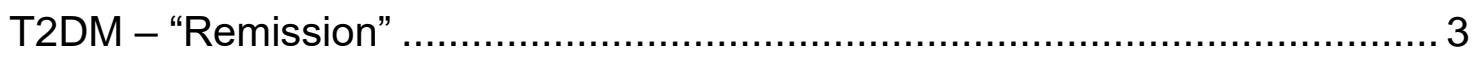

Study Aims and Hypotheses .............................................................. 4

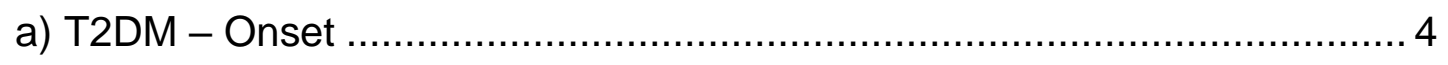

b) T2DM - Rate of "Remission" .............................................................. 4

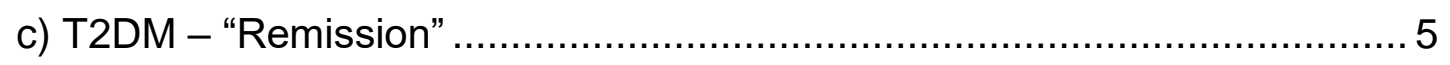

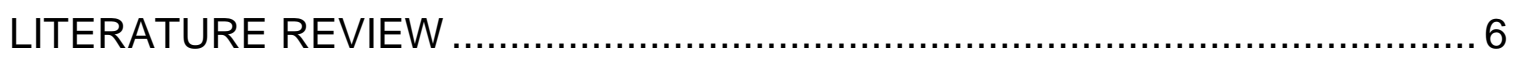

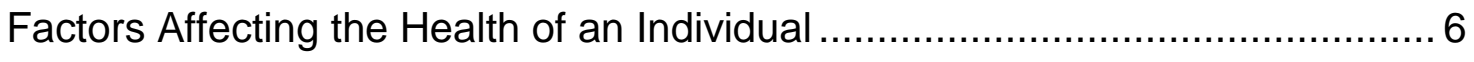




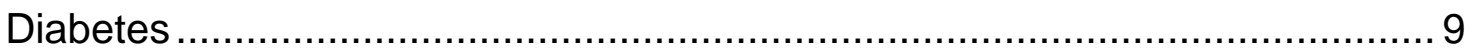

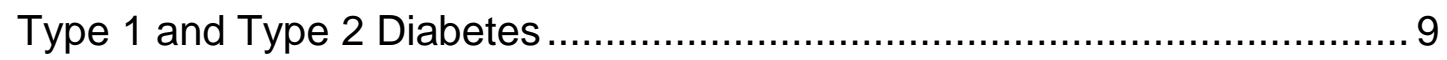

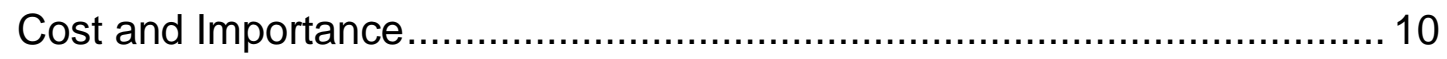

T2DM is a Progressive Disease Condition ............................................... 10

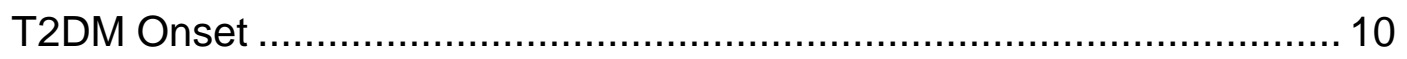

T2DM Onset and Progression - The Development of Complications....... 13

T2DM Onset Followed by “Remission” .................................................. 16

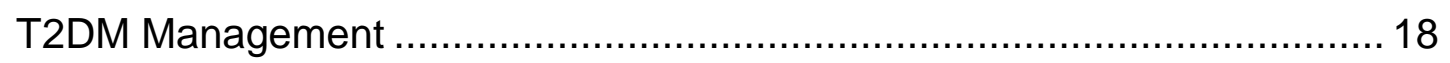

T2DM - Disease Management Programs .................................................. 21

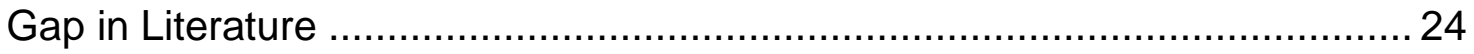

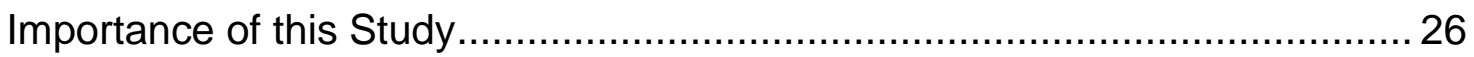

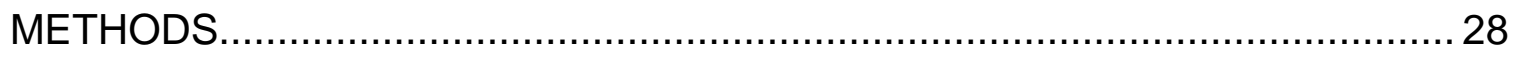

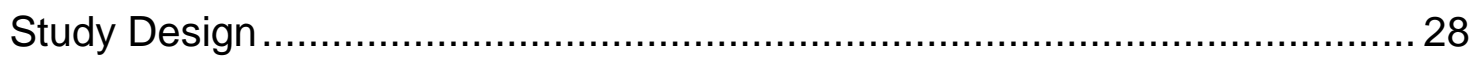

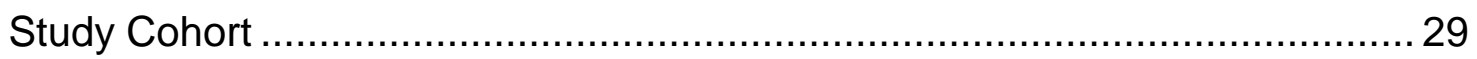

Data Aggregation - Steps, Inclusions and Exclusions ............................... 30

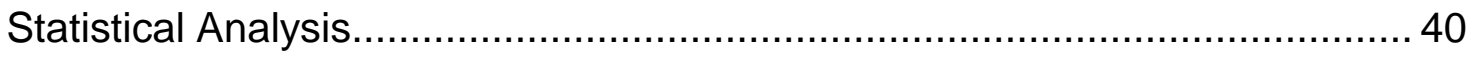

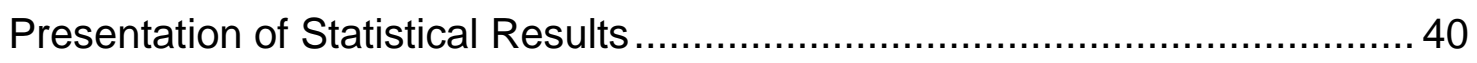

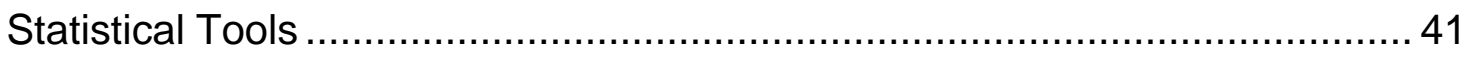


TYPE 2 DIABETES MELLITUS (T2DM) ONSET IN MEDICARE PATIENTS 65

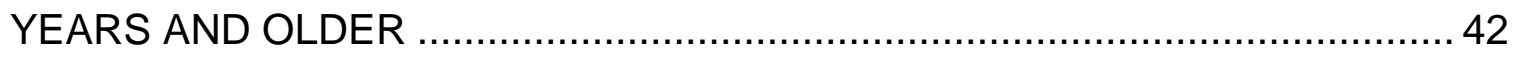

Research Design and Methods ............................................................. 43

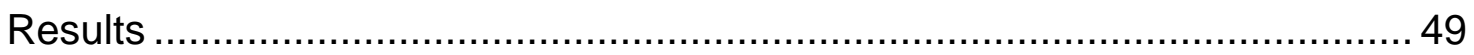

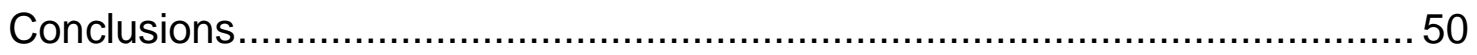

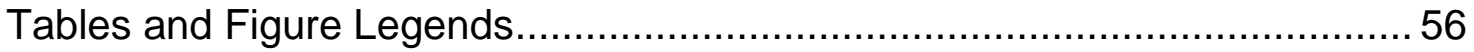

TYPE 2 DIABETES MELLITUS (T2DM) "REMISSION" IN MEDICARE PATIENTS 65 YEARS AND OLDER WITHOUT BARIATRIC SURGERY......... 64

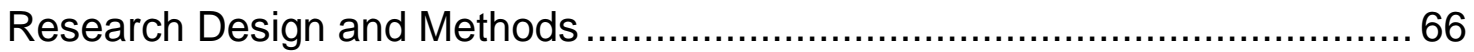

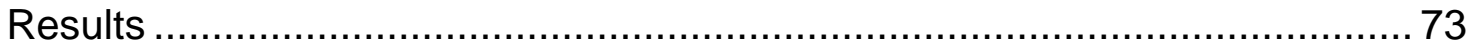

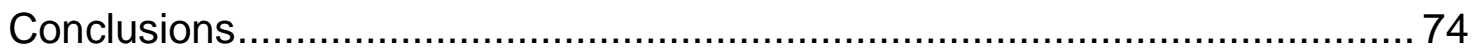

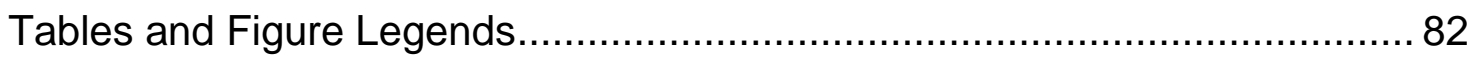

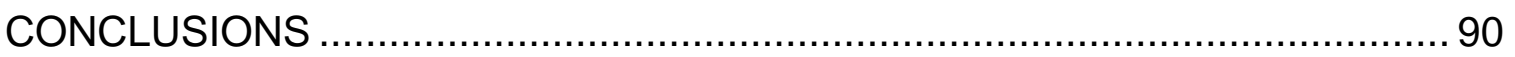

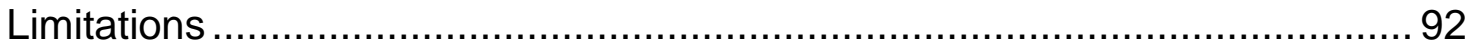

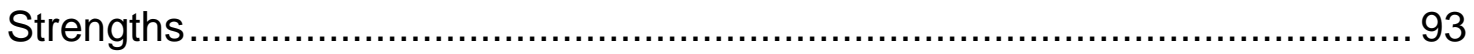

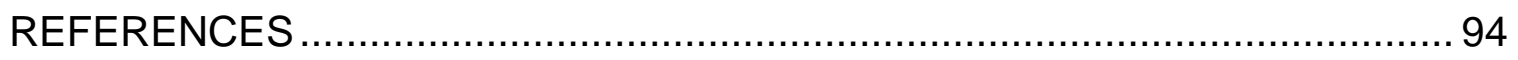

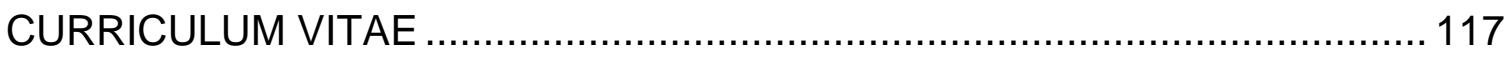




\section{LIST OF TABLES}

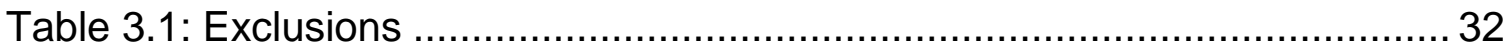

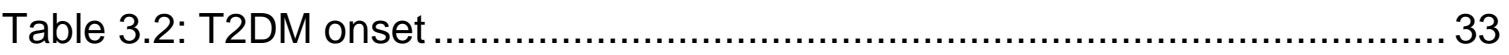

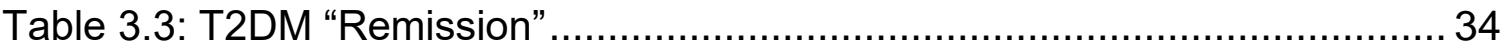

Table 3.4: Sample dataset - counting process input style.............................. 38

Table 3.5: Topics and example data elements included in the analytical data ... 39

Table 4.1: Baseline demographic and T2DM onset characteristics ...................56

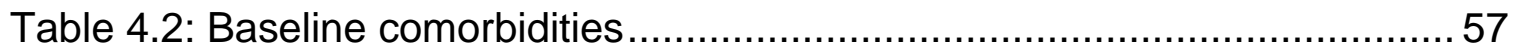

Table 5.1: Baseline demographic and T2DM "remission" characteristics ........... 82

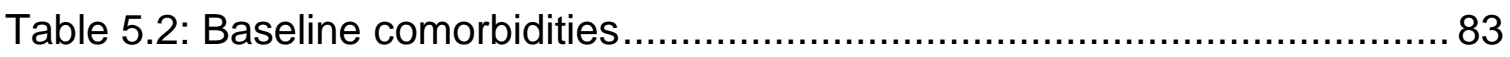




\section{LIST OF FIGURES}

Figure 2.1: Factors effecting the health and wellness of an individual ................ 8

Figure 2.2: Illustration of the natural progression of T2DM .............................. 12

Figure 2.3: Channels through which wellness programs are offered ................. 22

Figure 2.4: Test and learn cycle of a wellness program implementation ............ 27

Figure 4.1: Unadjusted Cox proportional hazard for T2DM onset...................... 58

Figure 4.2: Adjusted Cox proportional hazard for T2DM onset.......................... 59

Figure 5.1: Unadjusted Cox proportional hazard for T2DM "remission" .............. 85

Figure 5.2: Adjusted Cox proportional hazard for T2DM "remission" .................. 86 


\section{INTRODUCTION}

\section{Background to the study}

Type 2 Diabetes Mellitus (T2DM) is a chronic condition wherein the beta cells in the body do not produce enough insulin - a hormone that regulates blood sugar - or the body does not use insulin well enough (also called insulin resistance), or there is complete absence of insulin production. T2DM is associated with deleterious macro- and microvascular outcomes $(1,2)$ and is the leading cause of significant morbidities such as kidney failure, lower limb amputations, blindness (3). T2DM is the seventh leading cause of death in the United States and affects 29.1 million people (4). The total estimated national cost of diabetes in the United States in 2012 was more than $\$ 245$ billion, up from \$174 billion in 2007 (5).

T2DM is a progressive disease condition. If left unmanaged the T2DM progresses from onset (first occurrence of T2DM) to development of T2DM related complications and eventually death. However, when properly managed, in certain individuals the T2DM disease progression can be slowed down and in certain individuals T2DM "remission" is observed (i.e., no T2DM symptoms after onset - detailed definitions below in the literature review section below). 
Even though there is some available literature on factors associated with T2DM onset and "remission" there is a lot of opportunity to further understand these factors, especially in the Medicare population who are 65 years or older. Understanding the factors that impact T2DM disease progression is important because it will help in designing efficient interventions and/or help in creating better disease management and wellness programs. In this regards, the following study has been done to better understand the T2DM disease progression; specifically onset and "remission" of the T2DM, using administrative claims data, with three objectives as mentioned below.

\section{T2DM - Onset}

The first objective of this study is to understand the factors that predict the onset of T2DM. Previous studies have identified several risk factors associated with onset of T2DM. Some of these risk factors were family history of T2DM, age, obesity, and physical inactivity, prior history of gestational diabetes, impaired glucose tolerance, dipocytokines, inflammatory factors and hepatocyte factors, race/ethnicity, alcohol consumption, smoking/chewing tobacco, diet (6-11), high blood pressure (12), appendicectomy (13), and hypothyroidism (14). However, there is still additional opportunity to further understand these and other factors that are associated with the onset of T2DM, especially in the Medicare 65 years and older population. One example of this opportunity is to understand the demographic, clinical and pharmacological factors that are associated with onset of T2DM. 
It is important to understand the factors that are associated with the onset of T2DM because T2DM has a long asymptomatic phase (15). People can sometimes be unaware that they have diabetes for 4 to 7 years before an actual diagnosis $(16,17)$. Hence understanding these factors will help design better T2DM prevention program and help in early intervention. Even in cases where T2DM is identified later in its progression (due to late diagnosis), improved glycemic control is still beneficial and improves quality of life (18).

\section{T2DM - "Remission"}

The second objective of this study is to evaluate rate of T2DM "remission" in Medicare patients. The third objective of this study is to understand the factors that are associated with "remission" of T2DM. Even though it's rare, T2DM "remission" does occur in population without bariatric surgery $(19,20)$. However, there is very little available literature around the factors that are associated with "remission" among individuals with T2DM, who received usual care in Medicare 65 years and older population.

It is important to understand the factors associated with T2DM "remission" because T2DM is a progressive condition (21-23), i.e., if left unmanaged, as T2DM progresses the body's natural ability to regulate blood sugar decreases. In many patients the metabolic abnormalities associated with increased blood sugar (or hyperglycemia) leads to macrovascular (coronary artery disease, peripheral arterial disease, and stroke) and microvascular (diabetic nephropathy, neuropathy, and retinopathy) complications $(2,24,25)$. Understanding the factors 
associated with "remission" will help design better T2DM management programs.

In summary, the study presented herein is aimed to enhance our understanding about T2DM disease progression, to in turn help identify ways to design better interventions. In order to achieve this, the study focuses on the following three aims and hypotheses.

\section{Study Aims and Hypotheses}

\section{a) T2DM - Onset}

Aim 1: What are the factors that predict T2DM onset?

$\mathrm{H}_{0}$ : The sociodemographic and clinical profile of Medicare patients 65 years and older, who have T2DM onset, are not statistically different from those who do not have T2DM onset.

$\mathrm{H}_{\mathrm{A}}$ : The sociodemographic and clinical profile of Medicare patients 65 years and older, who have T2DM onset, are statistically different from those who do not have T2DM onset.

\section{b) T2DM - Rate of "Remission"}

Aim 2: What is the rate of T2DM "remission?"

Ho: T2DM Medicare patients 65 years and older, who received usual care without bariatric surgery, never achieve T2DM "remission."

$\mathrm{H}_{\mathrm{A}}$ : "Remission" is possible in T2DM Medicare patients 65 years and older, who received usual care without bariatric surgery. 


\section{c) T2DM - "Remission"}

Aim 3: What are the factors that predict T2DM "remission?"

$\mathrm{H}_{0}$ : The sociodemographic and clinical profile of Medicare patients 65 years and older, who have T2DM "remission", are not statistically different from those who do not have T2DM "remission."

$\mathrm{H}_{\mathrm{A}}$ : The sociodemographic and clinical profile of Medicare patients 65 years and older, who have T2DM "remission", are statistically different from those who do not have T2DM "remission." 


\section{LITERATURE REVIEW}

\section{Factors Affecting the Health of an Individual}

The United States (U.S.) spent nearly $\$ 3.0$ trillion, or $\$ 9,523$ per person in 2014 on health care costs, which was about 17.5 percent of the gross domestic product (GDP) (26). The U.S. is projected to spend nearly $\$ 5.4$ trillion, or 19.6 percent of GDP by 2024 on health care costs (27). At its current growth rate, spending on health care can adversely affect funding to other areas such as education, research and development, infrastructure, defense, and other public goods and services $(28,29)$. The U.S. spends more money per member per year on health care than any other country. Despite such costs, the U.S. ranks lower on key health measures such as life expectancy at birth, amenable mortality, etc., than other comparable nations $(30,31)$, which suggests that spending on health care does not always reflect quality of health (32).

Prior studies have demonstrated that health care accounts for only 10$20 \%$ of variability in health outcomes, while $80-90 \%$ of the variability in health outcomes results from other determinants such as individual behavior $(40 \%)$, socioeconomic factors (20\%) and genetics (30\%) (33-35). 
These factors (or determinants of health) that either directly or indirectly affect the health and well-being of an individual can be broadly categorized into the following groups:

- Health care: Includes sub-components such as access to health care, quality of health service and cost of health care services;

- Health behavior: Health behavioral factors are often modifiable and include factors such as level of engagement in one's own health, motivation to walk, diet, physical activity, tobacco consumption, alcohol consumption, participation in a wellness program, participation in a disease management program and managing stress;

- Other factors: Includes factors that can or cannot be modified by an individual: such as social (factors such as relationships with family and friends); economic (factors such as income, net worth); political (factors such as government policies, community); current health status of an individual (factors such as disabilities, current clinical conditions); biological (factors such as genetic); and, physical environmental (factors such as urban/rural, pollution) determinants (36) (Figure 2.1). 
Figure 2.1: Factors effecting the health and wellness of an individual

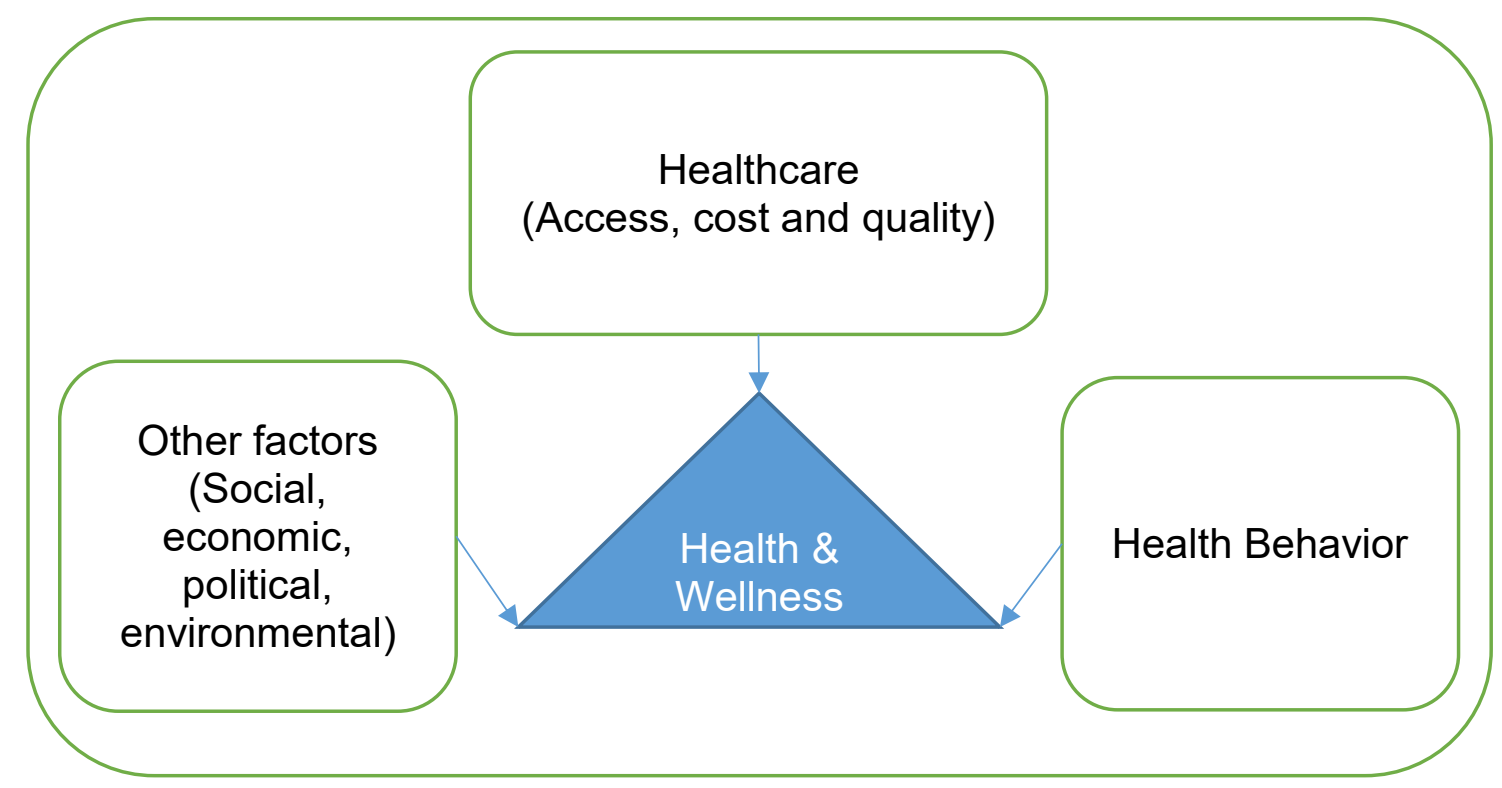

Therefore health of an individual is affected by multiple factors, often interacting with each other.

\section{Health Determinants and Chronic Conditions}

Chronic conditions like cancer, stroke, diabetes, heart disease, respiratory diseases etc., are among the leading causes for death, morbidity and disability in the U.S. (37). Chronic conditions account for 70 percent of deaths among Americans (38) and 75 percent of the cost of health care (39). Most of these chronic conditions are highly associated with factors such as: diet; physical activity; alcohol and tobacco consumption; social; and, environmental (40-44).

Chronic diseases are costly yet often preventable. In order to alleviate the burden of chronic conditions, public health and health care systems must design interventions to address these risk factors. These interventions will help in early 
detection, slow or stop progression of the chronic condition and reduce complications. This will in turn help in improving the quality of life and reduce the cost burden on health care and public health systems (45).

The first step in designing an effective disease intervention is to understand the factors that impact the onset of a chronic condition. This understanding will not only help designing effective comprehensive disease management and wellness programs but also help providers with a better understanding about its prognosis and treatment which, will in turn aid in disease counseling, and management.

\section{Diabetes}

\section{Type 1 and Type 2 Diabetes}

Diabetes is a chronic metabolic condition in which the body has a decreased ability to secrete insulin, a defect in insulin action or both (46). The two main etiopathogenetic categories of diabetes are type 1 and type 2 diabetes. Type 1 diabetes is an auto-immune disease that is characterized by an absolute lack of insulin secretion. Type 1 diabetes occurs in 5 to 10 percent of diabetes cases (47).

The second and more prevalent category of diabetes is type 2 diabetes mellitus (T2DM). T2DM is sometimes also referred as "non-insulin-dependent diabetes" or "adult-onset diabetes." T2DM accounts for 90 to 95 percent of diabetes cases (48) and is primarily characterized by worsening of insulin resistance and decline in beta cell (or $\beta$-cell) function. Beta cells produce and secrete insulin - the hormone that regulates blood glucose levels. This glucose 
imbalance can have a detrimental effect on how the body functions and can lead to other comorbidities (more details in the sub section below). This literature study focused on diagnosed T2DM. It excluded type 1 diabetes.

\section{Cost and Importance}

T2DM is the seventh leading underlying cause of death (49). Diagnosed T2DM accounts for nearly 10 percent of the cost of health care dollar (50). In 2012, the total economic cost of T2DM was $\$ 245$ billion dollars in the U.S. The major component of this cost was hospitalization ( $43 \%$ of the total medical cost). The medical expenses of people with diagnosed T2DM are 2.3 times that of a non-diabetics (5). Worldwide, an estimated 336 million have T2DM and it is projected that by 2030 nearly 552 million people will have T2DM (51).

\section{T2DM is a Progressive Disease Condition}

\section{T2DM Onset}

T2DM can have a long asymptomatic phase (15). People often are unaware that they have T2DM for 4 to 7 years before an actual diagnosis $(16,17$, 52). The onset of T2DM is generally preceded by a condition known as prediabetes (53). Pre-diabetes is a condition when the blood sugar is higher than normal, but not high enough to be classified as T2DM (54). Without intervention, pre-diabetes will most likely lead to the development of T2DM (55).

In this document the term "T2DM onset" refers to the onset of T2DM and not the onset of pre-diabetes. The term "T2DM onset" is used when an individual is diagnosed with T2DM for the first time during study period. 


\section{Risk factors - Onset}

Previous studies have identified several risk factors associated with the onset of T2DM such as physical inactivity, alcohol and tobacco consumption, diet, family history of diabetes, aging, obesity, prior history of gestational diabetes, impaired glucose tolerance, race/ethnicity, (6-11, 56-58), high blood pressure (12), appendicectomy (13), hypothyroidism (14), depression and anxiety (59). Pharmacological factors such as the use of statins also increases the risk of T2DM by $10-12 \%(60)$, statins have a dose dependent class effect on new-onset T2DM, i.e., the risk of developing T2DM increases with the increase in statin dose (61). Previous studies have hypothesized that statins alter the glucose homeostasis thereby causing T2DM onset (62). The speed of T2DM progression depends on the age of the first diagnosis, the earlier the onset of diabetes the more aggressive the progression or development of comorbidities $(56,63)$.

Several of these risk factors were previously identified using clinical trials and/or case control studies and might not be generalizable to community settings. Also, some of these risk factors are not specific to late onset of T2DM, especially in the 65 years and older population. Therefore, there is additional opportunity to understand factors associated with T2DM progression. There is not much literature available on the role of clinical conditions (such as retinopathy, neuropathy, peripheral vascular disease and cardiovascular disease) and demographics on T2DM onset and remission in T2DM Medicare patients 65 years or older (Figure 2.2). 
Figure 2.2: Illustration of the natural progression of T2DM

T2DM is a progressive disease. Glucose is a continuous variable, the use of thresholds such as pre-diabetic, T2DM is somewhat arbitrary (64). The speed and direction of progression depends on several factors as mentioned in the next sections.

Modifiable risk factors that increase the rate of progression

(such as sedentary lifestyle, obesity, diet)

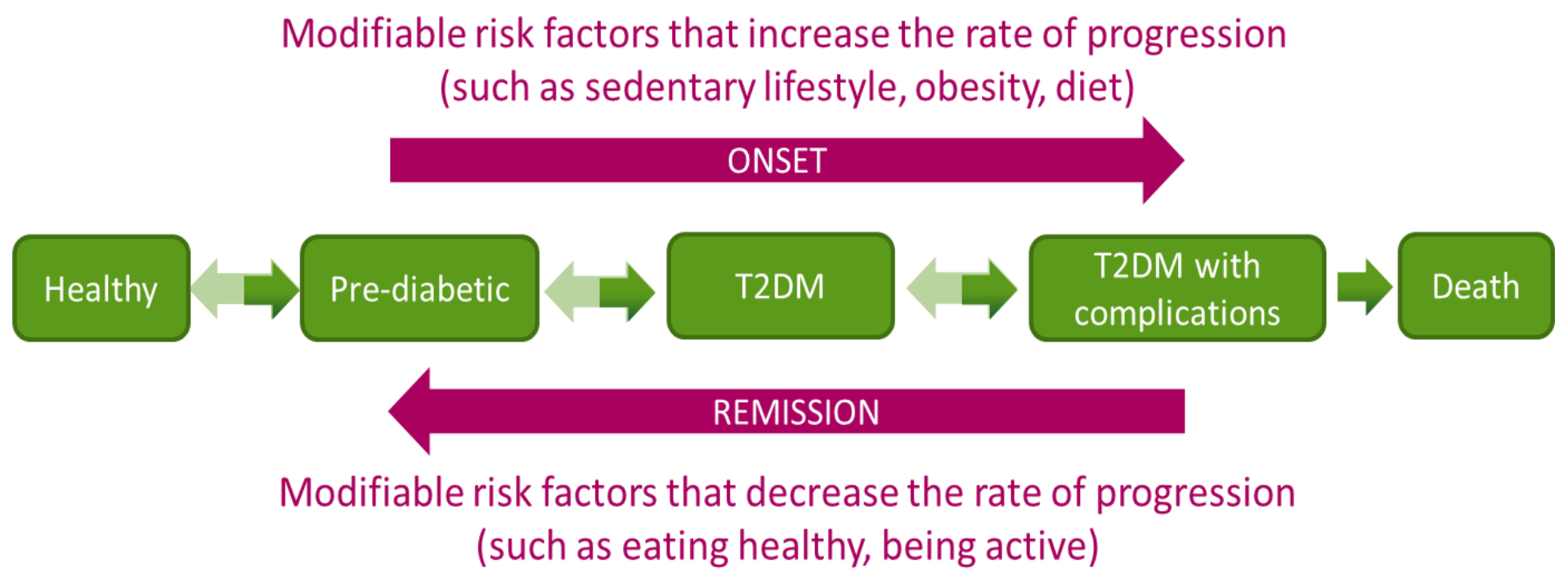




\section{T2DM Onset and Progression - The Development of Complications}

T2DM is a progressive condition. As T2DM progresses, the beta cells gradually lose their ability to produce insulin, which then progresses to beta cell failure and thereby requires insulin replacement (65). Long standing glucose imbalances is a key risk factor for macrovascular complications such as peripheral arterial disease (66), cerebrovascular (67), cardiovascular disease (68-70) and microvascular complications such as neuropathy, retinopathy and nephropathy $(24,64,71-73)$. T2DM is also associated with other clinical conditions such as dyslipidemia (74), hypertension (75), accelerated cognitive aging (76), breast cancer, colon cancer, pancreatic cancer, other types of cancer (77), mental health such as depression, anxiety, eating disorder (78), Parkinson's disease (79), disabilities (80) and mortality (81). These complications/comorbidities (or simultaneous presence of other disease conditions) are strongly associated with T2DM progression (64). Depending on the number and stage of the complications, these pathologies can cause significant disruption to the function of organs and body systems and thus effect quality of life (82). These complications and the perceived ability to control the disease, impacts the overall quality of life $(83,84)$. Hence the amelioration and prevention of these complications is important and has been a goal of major research in the recent years (85). 


\section{Complications associated with T2DM}

Hyperglycemia causes damage to vascular system. T2DM causes both macrovascular (hypertension, hyperlipidemia, cardiovascular, cerebral vascular disease, and peripheral vascular disease) and microvascular diseases (retinopathy, nephropathy, and neuropathy) (86). This section discusses the key vascular complications associated with T2DM.

Diabetic retinopathy: Retina is one of the most vascular regions in the human body. The damage due to hyperglycemia can vary depending on the severity of damage to the vascular system ranging from blurry vision to compete blindness. Nearly $21 \%$ of patients diagnosed T2DM have retinopathy at the time of onset (87).

Diabetic nephropathy: Diabetic nephropathy is also one of the important vascular complications and a major cause of chronic kidney disease (88).

Diabetic neuropathy: Neuropathy causes loss of sensation in the feet, which in turn causes callous formation. Neuropathy is associated with foot ulcers, nonhealing skin wounds and ulcers (89).

Cardiovascular diseases: Cardiovascular diseases associated with T2DM are the primary cause of mortality and morbidity. This is the primary reason why T2DM is the seventh largest cause of death in the United States (3).

Cerebrovascular: Cerebrovascular disease in T2DM is mainly because of atherosclerosis. The brain vessels disease can cause cognitive impairment and 
is a risk factor for dementia, Alzheimer's disease, transient ischemic attack (TIA) and stroke (90).

Peripheral Vascular Disease (PVD): The key factors for PVD are vascular smooth muscle cell dysfunction, inflammation, hypercoagulability and endothelial dysfunction (91). PVD primarily involves aneurysm and claudication.

Other complications mentioned above such as cancers, cognitive aging, death, are beyond the scope of this literature review.

\section{Metabolic Syndrome and T2DM}

Metabolic syndrome is characterized by a group of risk factors that raises the risk of heart disease and other health problems such as stroke or diabetes. These risk factors include large waistline, high triglyceride level, low HDL cholesterol level, high blood pressure, high fasting blood sugar $(92,93)$. Patients with metabolic syndrome are at a higher risk of developing T2DM by threefold and the risk of developing cardiovascular diseases by two fold (94-96). This study does not use metabolic syndrome as a predictor or classifier, due to the lack of $\mathrm{HbA} 1_{\mathrm{c}}$ data and glucose data.

\section{Diabetes Complications Severity Index (DCSI Score)}

The severity of the above mentioned T2DM complications varies from one patient to another. Therefore the severity of the disease varies depending on the stage of diabetes (pre-diabetes vs. T2DM) and/or the presence of T2DM related complications. An individual with pre-diabetes and no complications is considered having the least severity due to T2DM, while a T2DM patient with 
multiple complications would be considered having the most severity due to T2DM (more details below). Individuals who die due to T2DM related complications theoretically have the most severe complications, but are excluded in the study presented below.

The complications associated with T2DM usually develop simultaneously or consecutively rather than independently. Hence a simple count of complications will not provide sufficient measure of T2DM disease progression and severity. Therefore an indicator (or score) was previously developed to quantify the severity of diabetic complications $(97,98)$. This indicator is called the Diabetes Complications Severity Index (DCSI) and models the severity of diabetes complication at any given point of time in the patient's life. DCSI can hence be used to access patient's risk of adverse outcomes (like hospitalization or death). Administrative claims (medical, pharmacy and lab data) are used to calculate DCSI. This score was subsequently validated (99) and adopted by other researchers (100-104). This study uses DCSI scores to quantify T2DM diabetes severity.

\section{T2DM Onset Followed by "Remission"}

T2DM disease progression is generally regarded as irreversible (105). However with certain types of interventions such as bariatric surgery, metabolic surgery, lifestyle efforts (e.g., weight loss and exercise), in certain individuals T2DM "remission" is observed. This literature review excluded remission due to bariatric/metabolic surgery because the focus of the study was to understand the factors associated with remission in a non-surgery based setting. 


\section{Remission - Definition}

The American Diabetes Association (ADA) has proposed the following definitions for T2DM remission (106).

a) Partial remission: Partial remission is defined as sub-diabetic hyperglycemia without active pharmacologic therapy or ongoing procedures for at least 1 year. Sub-diabetic hyperglycemia is when $\mathrm{HbA} 1_{c}<6.5 \%$ and/or fasting glucose 100 $125 \mathrm{mg} / \mathrm{dL}[5.6-6.9 \mathrm{mmol} / \mathrm{l}]$.

b) Complete remission: Complete remission is defined as "normal" measures of glucose metabolism without active pharmacologic therapy or ongoing procedures for at least 1 year. Normal measure of glucose metabolism is when $\mathrm{HbA} 1_{\mathrm{c}}$ in the normal range, $<5.7 \%,<39 \mathrm{mmol} / \mathrm{mol}$, fasting glucose $<100 \mathrm{mg} / \mathrm{dl}[5.6 \mathrm{mmol} / \mathrm{l}])$

c) Prolonged remission: Complete remission for at least 5 years.

Since, T2DM is a chronic disease and individuals who go into remission are at a high risk of relapse, the term cure is not used by ADA for even prolonged remission because cure is generally the restoration of health, whereas remission is the abatement of signs and symptoms of disease (106).

\section{Risk factors - Remission}

Current understanding about T2DM remission due to non-bariatric intervention is limited. The recent post hoc randomized controlled study from the Look AHEAD (Action for Health for Diabetes) and the 2014 Kaiser Permanente study suggest that factors such as age, race, time since diagnosis, baseline $\mathrm{HbA} 1_{\mathrm{c}}$ level and taking diabetes medication at baseline are associated with 
T2DM remission $(19,20,107)$. There is also limited information on how T2DM remission occurs in a usual care setting especially in the Medicare patients 65 years or older.

\section{T2DM Management}

The American Diabetes Association's (ADA's) "Standards of Medical Care in Diabetes - 2016" provided details on several components of T2DM management, care, general treatment goals, and tools to evaluate the quality of care (108). Reviewing these factors for the study is important because an individual who is managing their T2DM will favorably alter the disease progression (109). Therefore this section of the literature review highlights some of the key T2DM management and interventions.

Treatment of T2DM generally includes lifestyle interventions and/or medical interventions (or non-lifestyle related diabetes management).

\section{T2DM - Lifestyle Interventions}

Lifestyle interventions of diabetes generally include education, nutritional therapy, blood sugar monitoring and physical activity.

Education: The first and probably the most important component of diabetes management is patient education, i.e., make the patient aware of the disease condition (110). Such educational programs may include information about the complexity of diabetes, its potential impact if left unmanaged, information on healthy eating, methods to stay physically active, etc. Education is effective in improving outcomes related to diabetes (111-116). However, there is no "one 
size fits all" educational program for diabetics. At a macro level, the role of education is well established, for example, it is demonstrated that education programs that help in modifying behavioral and psychosocial aspects have improved health outcomes (117-119). However, when designing, testing and implementing an education program at a micro level to a particular diabetic individual or group, several other variables such as trust on the source of education material, leadership, partnership, might also be important (120).

Nutrition: Nutrition is a key component of diabetes management. However, as with education, there is no single nutritional strategy that works the best for all diabetics (121). A nutritional management program is designed to motivate individuals with T2DM to make healthy food choices.

Physical activities: Regular physical activities reduce the risk of diabetic progression (122). American Diabetes Association recommends 90 minutes of vigorous physical activity or 150 minutes of moderate physical activity per week. As cardiorespiratory fitness and physical activity increases, the associated health benefits also increase proportionally (123-125).

Previous participation in physical activities were difficult to quantify unless under study conditions. However with the advent of technology, numerous devices such as pedometers, smart watches etc. are now available that can accurately and passively capture some of the physical activity data and information. 


\section{T2DM - Medical Intervention - Pharmacy}

Medical intervention can be in the form of pharmaceuticals such as oral anti-diabetic medications and/or injectable therapies such as insulin. Providers use several different types of T2DM medication. These medications might be used individually or in combination, depending on requirement and may include monotherapy e.g., use of metformin, dual therapy e.g., metformin $+1^{\text {st }}$ line agent like Alpha-glucosidase inhibitors, triple therapy e.g., metformin $+1^{\text {st }}$ line agent $+2^{\text {nd }}$ line agent like Colesevelam, or insulin, etc. $(126,127)$.

\section{T2DM - Medical Intervention - Surgery}

In certain obese individuals with T2DM, medical intervention may also include medically assisted weight loss, using surgery. Weight loss is generally considered first in T2DM disease management (128), especially in obese T2DM patients. Obese T2DM patients with sustained weight loss have been shown to improve glycemic control. In certain patients weight loss can be achieved by intervention strategies such as diet, physical activity, behavioral therapy, lifestyle intervention, pharmacological therapy, bariatric ("weight-loss") surgery and other metabolic surgeries such as gastric banding (or procedures that involve resecting, bypassing or transposing sections of the stomach and small intestine). Bariatric surgery commonly includes gastric bypass such as Roux-en-Y-gastric bypass (RYGBP), sleeve gastrectomy such as Laparoscopic Sleeve Gastrectomy, adjustable gastric band, and biliopancreatic diversion with duodenal switch (BPD/DS) (129). Bariatric and metabolic surgeries are often 
considered as an effective weight loss treatments for certain individuals with severe obesity especially amongst adults with BMI $\geq 35 \mathrm{~kg} / \mathrm{m} 2$.

Bariatric surgery has been proved to be effective in treating T2DM patients whose BMI is $35 \mathrm{~kg} / \mathrm{m} 2$ or higher (130) with a success of achieving T2DM remission between $24 \%$ and $95 \%(131,132)$. Bariatric surgery is different from lifestyle intervention because not every individual qualify for a bariatric surgery, also the mechanism for remission after bariatric surgery is still unclear and might be different from the mechanism of remission following lifestyle intervention (132). Therefore individuals with bariatric surgery were excluded from the analysis.

\section{T2DM - Disease Management Programs}

\section{Wellness Programs}

Wellness programs utilize education (133), behavior modification (134), counseling (135), and incentives (136), etc., to help make better health and wellness choices. Wellness programs are generally offered either through workplace (called workplace wellness programs) or directly to individuals (e.g., self-employed, Medicare members, etc.) (137) (Figure 2.3). 
Figure 2.3: Channels through which wellness programs are offered

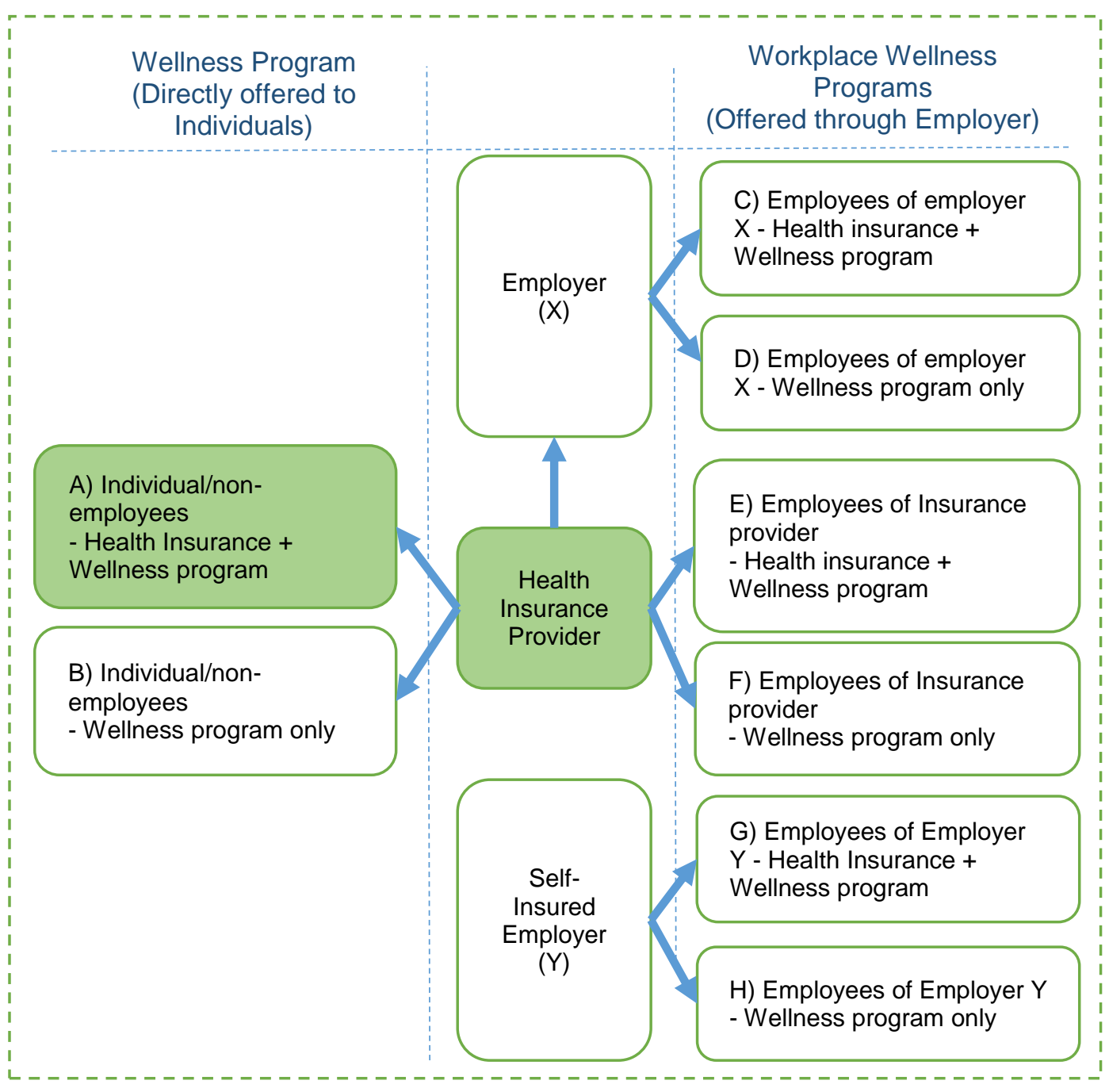

The current study focused only on "A) Individual/non-employees - Health

Insurance + Wellness program." 
Lifestyle interventions are a major component of wellness programs that are offered by health insurance companies to their members (or policy holders). These wellness programs are generally offered as an add-on to the health insurance coverage for members. A wellness program could have multiple "lifestyle intervention" related components such as education, diet, promoting physical activity, campaigns related to medication adherence, annual health assessments, etc. (138). Each "lifestyle intervention" component could have subcomponents. For example, the component "promoting physical activity" might include sub-components like promoting walking, gym and yoga, $5 \mathrm{k}$ events for encouraging running. Some members might prefer walking and some might prefer going to a gym, while some might prefer a combination of these activities. Wellness programs are generally offered in multiple formats to provide a "variety of choices" to the participants and to foster those with preferential participation. This study presented herein does not differentiate the type of lifestyle intervention and only considered any non-bariatric lifestyle intervention.

\section{Disease Management Programs}

Health insurance providers also have several disease management programs which are separate from the wellness programs. These disease management programs are designed specifically for each disease and often provided in addition to wellness programs. For example, the diabetes disease management program might provide clinical guidance to their members who are pre-diabetic or diagnosed with T2DM. 


\section{Gap in Literature}

A well designed lifestyle intervention; either as a standalone program or as part of a comprehensive wellness program or T2DM disease management program, includes components such as diet, exercise, education/counseling, smoking cessation, behavior modification (139). Some of these lifestyle interventions are hypothesized to achieve key outcomes such as decreased health care cost, improved quality of life, improved health outcomes, stop or delay the onset of T2DM, and stop or delay progression of T2DM complications $(110,140)$. It is also noted that a single intervention will not be able to address all the above factors at the same time, and might need multiple lifestyle interventions at various phases of T2DM disease progression.

Some well-designed lifestyle intervention programs aimed at individuals with T2DM have shown positive health outcomes (125, 141-143). Some lifestyle intervention programs work, while some do not (144). Within those that work, their level of success varies greatly from one program to another. Success of these lifestyle intervention programs depend largely on whose perspective the program is being implemented and may include: stakeholder (provider, insurance agency, self); the goals of the program (to make money vs. keep individuals healthy); program design (components/services offered in the lifestyle intervention program); implementation (level of activation of the program, incentives, penalties); and most importantly how the program is evaluated (quality of life, unhealthy days, absenteeism). An effective lifestyle intervention program is highly customized and engages the intended audience at an 
"individual level." In order to achieve high level of customization, it is imperative to understand questions such as: "what works?"; "how to engage?" and, "what needs to be improved?" The study presented here does not evaluate all these questions but focusses only on understanding the factors that predict T2DM onset and "remission."

Lifestyle interventions applied as a community based strategy are not effective and need to be tailored (145). The first step in designing a comprehensive lifestyle intervention programs for T2DM is to understand T2DM progression. T2DM progression has the following components; a) before onset of T2DM (prevention), factors associated with onset of T2DM; b) after onset of T2DM (treatment/management), factors associated with progression and factors associated with "remission."

\section{T2DM Onset}

As discussed above, there is still a gap in the literature about the association of clinical conditions (such as retinopathy, neuropathy, peripheral vascular disease and metabolic disease) and demographics with the onset of T2DM in Medicare patients 65 years and older.

\section{T2DM “Remission”}

There are unanswered questions about the role of lifestyle interventions in T2DM "remission." For example, what are the demographic, clinical factors that are associated with individuals who have T2DM "remission" without bariatric surgery? 
Even though lifestyle intervention is considered more effective than medical intervention for pre-diabetic patients and patients with impaired glucose tolerance (146-148), the role of lifestyle interventions for T2DM "remission" is still unclear. Though there is already some evidence for the possibility of T2DM "remission" outside bariatric surgery, the rate of incidence is relatively low. Hence there is value in understanding the demographic, pharmacological and clinical factors that are associated with T2DM "remission" without bariatric surgery.

In summary, the purpose of the study is to evaluate the following two questions

1. Progression to Onset: What are the factors that predict T2DM onset?

2. Progression to "Remission": What are the factors that predict T2DM "remission?"

\section{Importance of this Study}

a) Monetary Impact: Potential saving of $\$ 3$ to $\$ 4.6$ billion in savings annually in Kentucky (149).

b) Quality of life: More healthy and happy days due to reduced morbidity.

\section{c) The need for efficient intervention:}

Stakeholders such as health care systems, providers and payors do not have unlimited resources to use blanket approaches for intervention. Organizations are using data analytics to help design efficient lifestyle intervention programs. Data mining can provide valuable and actionable insights. These insights can be used to design, test analyze, learn and revise specific hypothesis. This continuous "test and learn" process will help optimize the 
allocation of scarce resources to high potential wellness initiatives (Figure 2.4). This will in turn help target the right individual with the right intervention.

Figure 2.4: Test and learn cycle of a wellness program implementation

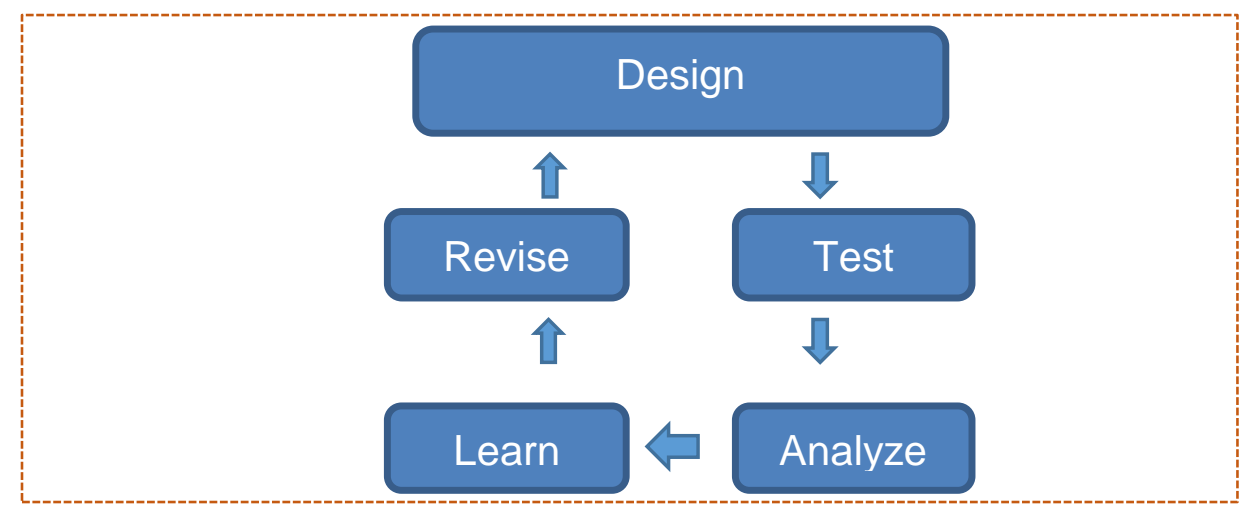

Also, rather than targeting the entire population for specific wellness component, predictive analytics (like predictive models, segmentation, clustering) of data can be used to create targeted high impact intervention to specific audiences, thereby increasing adoption and success of the intervention.

\section{d) Compression of Comorbidity}

Compression of comorbidity is to postpone the age of the first onset of chronic infirmity so that the lifetime illness burden is compressed into a shorted period toward the age of death (150). In order to achieve compression of morbidity, it is important to understand the prognosis of the disease and the factors that impact the progression. 


\section{METHODS}

\section{Study Design}

The study is a retrospective cohort analysis using administrative Medicare Advantage Prescription Drug (MAPD) health plan data from a large Managed Care Organization (MCO). This administrative data included enrollment, medical claims and pharmacy claims data. This data were collected during the standard course of care. It was not originally collected for the purpose of research or any new study of human or animal subjects. Prior to the start of the study this study protocol was reviewed and approved by the University of Louisville Institutional Review Board. This study was also reviewed by the MCO's review board.

\section{De-identification:}

The data was de-identified before analysis using industry standard deidentification process. The following are some of the example identifiers that were removed from the data as part of the MCO's de-identification process

a. Names.

b. All geographic subdivisions smaller than a state, including street address, city, county, precinct, zip Code, and their equivalent geographical codes. 
c. All elements of dates (except year) for dates directly related to an individual, including birth date, admission date, discharge date, date of death; and all ages over 89 and all elements of dates (including year) indicative of such age.

d. Telephone numbers, facsimile numbers, electronic mail addresses, social security numbers, medical record numbers, health plan beneficiary numbers, account numbers, certificate/license numbers, vehicle identifiers and serial numbers, including license plate numbers, device identifiers and serial numbers, web universal resource locators (url), internet protocol (IP) address numbers, biometric identifiers, including fingerprints and voiceprints, full-face photographic images and any comparable images.

e. Any other unique identifying number, characteristic, or code such as social media handles/profile names/ids, mac address, mobile device id number or mobile unique device ID.

\section{Study Cohort}

The federal government offers original Medicare insurance, which includes Medicare Part A (Hospital Insurance) and Medicare Part B (Medical Insurance). Part C (or Medicare advantage) is offered through private insurance companies and include the benefits of Part A, Part B and sometimes the optional Part D (Prescription Coverage) along with several choices of programs and services. Individuals can only be enrolled in Part C if they qualify for Medicare Part A and Part B.

The private MCO offers Medicare Advantage Prescription Drug Plan (MAPD) a Part C plan, which has the benefits of Medicare Part A, Part B and 
Part D, to individuals (Individual MAPD) and groups (Group MAPD). The source population for this study are individuals who enrolled (members) in MCO's individual MAPD plan. The steps taken to create the aggregated dataset are mentioned below.

\section{Data Aggregation - Steps, Inclusions and Exclusions}

The data aggregation was done in Hadoop (using Spark, Scala and Hive). Hadoop is an open source software framework for distributed processing and distributed storage of very large datasets (151). Processing was done in Hadoop because of the volume of the data. Means and frequencies were calculated in Hadoop. This data was then moved into SAS 9.2 (SAS Institute Inc., Cary NC) to calculate rate for T2DM onset and "remission."

Step1: Identify active enrollees: Identify active MAPD enrollees who were active with the MCO in 2015. There were $>2.4$ million individuals who met this criteria.

Step2: Include only Medicare age-ins: The study included only those members whose initial eligibility for Medicare enrollment was based on their age. An individual can qualify to receive Medicare benefits if they are at least 65 years in the year of their Medicare enrollment and meet certain additional enrollment criteria. Therefore this study excludes those people who qualify for Medicare for any other reasons such as disabilities or end stage renal disease (ESRD). There were 1.7 Million individuals who met this criteria. 
Step 3: Exclusions: Excluded individuals who did not have continuous Individual MAPD coverage with MCO from January 2008 to December 2015. This ensured that the inclusion of MAPD individual who had both medical and pharmacy coverage through the MCO continuously for eight years with the MCO. Individuals with bariatric surgery, polycystic ovary syndrome, lipodystrophy, nephropathy, type 1 diabetes, lived in Puerto Rico, steroid induced T2DM were excluded from the study. These conditions were identified using a combination of diagnostic codes, procedure codes, and therapeutic drug description. There were 121,862 individuals in the study cohort after this exclusion.

Step4: Identify T2DM: T2DM individuals were identified using administrative medical and pharmacy claims using the following criteria; at least two diagnoses of T2DM (International Classification of Diseases, Ninth Revision, Clinical Modification [ICD-9-CM] and/or (International Classification of Diseases, Tenth Revision, Clinical Modification [ICD-10-CM]; at least two pharmacy scripts related to T2DM; at least 2 claims (medical or pharmacy) related to T2DM. A minimum of two claims was used in the identification of T2DM because this would help exclude patients who were miscoded and patients who were suspected to have T2DM but were never formally diagnosed. T2DM status was identified for each year from 2008 to 2015.

Using eight years of data, each individual was assigned into a group based on their T2DM status over 8 years (Table 3.1). Each individual was assigned 8 character sequence to represent their T2DM status for each year from 2008 to 2015, where character at position 1 represents T2DM status of 
2008; position 2 represents T2DM status of 2009 and so on. So a sequence 00000000 represents an individual who had no diabetes in any year 2008 to 2015 and a sequence 00000011 represents an individual who was identified to have no T2DM from 2008 to 2013 and had a T2DM onset in 2014 and continued to have T2DM in 2015. These groups were then categorized into three cohorts: a) onset cohort; b) "remission" cohort; and, d) other cohort as described below.

Table 3.1: Exclusions

\begin{tabular}{|c|c|c|c|c|}
\hline T2DM & Sequence $\S \S$ & $\mathbf{n}$ & $\%$ & \\
\hline Always T2DM & 11111111 & 9,559 & $7.8 \%$ & Onset cohort \\
\hline No T2DM & 00000000 & 85,076 & $69.8 \%$ & "Remission" cohort \\
\hline "Remission" & $\begin{array}{l}11111110,11111100, \\
11111000,11110000\end{array}$ & 500 & $0.4 \%$ & "Remission" cohort \\
\hline Onset & $\begin{array}{c}00000001,00000011 \\
00000111,00001111\end{array}$ & 4,314 & $3.5 \%$ & Onset cohort \\
\hline $\begin{array}{l}\text { Newly diagnosed } \\
+ \text { "remission" }\end{array}$ & $\begin{array}{l}00000010,00000110 \\
00000100,00001100\end{array}$ & 2,330 & $1.9 \%$ & Excluded \\
\hline $\begin{array}{l}\text { Other } \\
\text { combinations }\end{array}$ & $X X X X X X X X$ & 20,083 & $16.5 \%$ & Excluded \\
\hline Total & & 121,862 & $100.0 \%$ & \\
\hline
\end{tabular}

$\S \S_{0}=$ no $\mathrm{T} 2 \mathrm{DM}$ and $1=$ presence of $\mathrm{T} 2 \mathrm{DM}$

\section{a) T2DM onset cohort}

In order to be included in the onset cohort, individuals were required to have no T2DM in the year 2008, 2009, 2010 and 2011. This condition enabled to establish a natural history of T2DM for at least four years. The occurrence of onset was evaluated in 2012, 2013, 2014 and 2015. Also, once an individual was diagnosed with T2DM, only those who continued to have T2DM in the subsequent years till the end of the study were considered to have a T2DM onset 
(Table 3.2). Individuals who did not have T2DM any year from 2008 through 2015 were considered as part of the no onset group (Table 3.2).

Table 3.2: T2DM onset

T2DM Diagnosis

\begin{tabular}{|c|c|c|c|c|c|c|c|c|c|}
\hline $2008 \S$ & 2009 & 2010 & 2011 & 2012 & 2013 & 2014 & 2015 & PATTERN§§ & Description \\
\hline NO & NO & NO & NO & NO & NO & NO & NO & 00000000 & $\begin{array}{c}\text { No Onset } \\
(n=85,076)\end{array}$ \\
\hline $\mathrm{NO}$ & $\mathrm{NO}$ & $\mathrm{NO}$ & $\mathrm{NO}$ & NO & $\mathrm{NO}$ & $\mathrm{NO}$ & YES & 00000001 & \\
\hline NO & NO & NO & NO & NO & NO & YES & YES & 00000011 & Onset \\
\hline NO & NO & NO & NO & NO & YES & YES & YES & 00000111 & $(n=4,314)$ \\
\hline NO & NO & NO & NO & YES & YES & YES & YES & 00001111 & \\
\hline $\mathrm{NO}$ & $\mathrm{NO}$ & $\mathrm{NO}$ & YES & YES & YES & YES & YES & 00011111 & Onset \\
\hline NO & NO & YES & YES & YES & YES & YES & YES & 00111111 & (Excluded) \\
\hline NO & YES & YES & YES & YES & YES & YES & YES & 01111111 & \\
\hline NO & $\mathrm{NO}$ & NO & $\mathrm{NO}$ & NO & $\mathrm{NO}$ & YES & NO & 00000010 & No continuous \\
\hline NO & NO & NO & NO & NO & YES & NO & YES & 00000101 & Onset (Excluded) \\
\hline
\end{tabular}

$\S \mathrm{NO}=$ no type 2 diabetes diagnosis, and YES = type 2 diabetes diagnosis $\S \S_{0}=$ no $\mathrm{T} 2 \mathrm{DM}$ and $1=$ presence of $\mathrm{T} 2 \mathrm{DM}$

The onset cohort had a total of 89,390 individuals. The censored variable was binary $(0,1)$. The value of 1 was assigned for individuals who had a T2DM onset $(n=4,314)$ and a value of 0 was assigned for individuals who did not have T2DM in the eight years of study $(n=85,076)$.

\section{b) "Remission" cohort}

The definition of "remission" in this study was different from that used by American Diabetes Association (ADA), because of the lack of $\mathrm{HbA} 1_{c}$ data. An individual was considered to be in "remission" if they had two or more T2DM related claims in previous years and had no T2DM related claim in the given year. Some individual's experience T2DM relapse followed by a year or two 
years of "remission." Such individuals were excluded from the study i.e., upon "remission" T2DM individuals were required to continue to have no T2DM related claims till the end of study period i.e., 2015. Also, to be included in the T2DM "remission" study cohort, individuals were required to have T2DM in the year 2008, 2009, 2010 and 2011 and either continue to be diabetic (no "remission" group) or go into "remission" ("remission" group). This condition enabled to establish a natural history of T2DM for at least four years. T2DM relapse can happen in some individuals after "remission". Those individuals who had relapse after first "remission" were excluded from the study, in order to focus on only those individuals who had continuous "remission" (Table 3.3).

Table 3.3: T2DM "Remission"

T2DM DIAGNOSIS

\begin{tabular}{|c|c|c|c|c|c|c|c|c|c|}
\hline $2008^{\S}$ & 2009 & 2010 & 2011 & 2012 & 2013 & 2014 & 2015 & PATTERN $\S \S$ & Description \\
\hline YES & YES & YES & YES & YES & YES & YES & YES & 11111111 & $\begin{array}{l}\text { No "Remission" } \\
(\mathrm{n}=9,559)\end{array}$ \\
\hline YES & YES & YES & YES & YES & YES & YES & NO & 11111110 & \\
\hline YES & YES & YES & YES & YES & YES & NO & NO & 11111100 & "Remission" \\
\hline YES & YES & YES & YES & YES & NO & NO & NO & 11111000 & $(n=500)$ \\
\hline YES & YES & YES & YES & NO & $\mathrm{NO}$ & $\mathrm{NO}$ & $\mathrm{NO}$ & 11110000 & \\
\hline YES & YES & YES & $\mathrm{NO}$ & NO & $\mathrm{NO}$ & $\mathrm{NO}$ & $\mathrm{NO}$ & 11100000 & "Remission" \\
\hline YES & YES & NO & NO & NO & NO & NO & NO & 11000000 & (Excluded) \\
\hline YES & NO & NO & NO & NO & NO & NO & NO & 10000000 & \\
\hline YES & YES & YES & YES & $\mathrm{NO}$ & $\mathrm{NO}$ & YES & YES & 11110011 & nission" + Relapse \\
\hline YES & YES & YES & YES & NO & YES & NO & YES & 11110101 & (Excluded) \\
\hline
\end{tabular}

$\S N O=$ no type 2 diabetes diagnosis, and YES = type 2 diabetes diagnosis $\S \S 0=$ no $\mathrm{T} 2 \mathrm{DM}$ and $1=$ presence of $\mathrm{T} 2 \mathrm{DM}$

The "remission" cohort had a total of 10,059 individuals. The censored variable was binary $(0,1)$. The value of 1 was assigned for individuals who had a 
T2DM "remission" $(n=500))$ and a value of 0 was assigned for individuals who had T2DM every year from 2008 to $2015(n=9,559)$.

\section{c) Other Cohort:}

Among the 121,862 individuals in the study frame there were $18.4 \%$ $(16.5 \%+1.9 \%)$ individuals who had T2DM combinations that did not meet our definition of onset or "remission" as mentioned above. The individuals were excluded from the analysis in order to account for only those individuals who continued to have the event (either onset or "remission") after its first occurrence and excluded relapses. This study could have included these $18.4 \%$ individuals in the analysis, but the behavior of individual who had a relapse is likely different from those who did not have a relapse, a choice was made to exclude them from this study.

Step5: Independent variables: After accounting for the inclusions and exclusions, the remaining members were included in the analytical dataset. These members are then tagged with additional data components. Primary topics and data components are mentioned below:

a) Clinical tags: Clinical tags were binary indicators (member had clinical condition $\mathrm{Y} / \mathrm{N}$ ) for each year from 2008 to 2015 . These clinical conditions are the key macrovascular and microvascular disease conditions that are commonly associated with T2DM. Administrative medical claims were used for identification of these clinical tags as described before (98). These clinical conditions include; retinopathy (diabetic ophthalmologic disease, background 
retinopathy, other retinopathy, retinal edema, cystoid macular edema/degeneration (CSME), other retinal disorders, proliferative retinopathy, retinal detachment, blindness, vitreous hemorrhage); nephropathy (diabetic nephropathy, acute glomerulonephritis, nephrotic syndrome, hypertension, nephrosis, chronic glomerulonephritis, nephritis/nephropathy, chronic renal failure, renal failure not otherwise specified, renal insufficiency); neuropathy (diabetic neuropathy, amyotrophy, cranial nerve palsy, mononeuropathy, Charcot's arthropathy, polyneuropathy, neurogenic bladder, autonomic neuropathy, gastroparesis/diarrhea, orthostatic hypotension) cerebrovascular (transient ischemic attack, stroke); cardiovascular (atherosclerosis, other ischemic heart disease, angina pectoris, other chronic IHD, myocardial infarction, ventricular fibrillation arrest, atrial fibrillation arrest, other atherosclerotic cardiovascular disease, old myocardial infarction, heart failure, atherosclerosis severe, aortic aneurysm/dissection); peripheral vascular disease PVD (diabetic PVD, other aneurysm in lower extremities, PVD, foot wound complication, claudication intermittent, embolism/thrombosis (lower extremity), gangrene, gas gangrene, ulcer of lower limbs); metabolic (ketoacidosis, hyperosmolar, other coma). Apart from these clinical tags, hypertension, hyperlipidemia were also included in the analysis.

The selection of these tags in the onset and "remission" cohort depended on the definition of the clinical tags. For example, clinical tags such as diabetic ophthalmologic disease (ICD 09 250.5x), Diabetic nephropathy (ICD9 250.4) were excluded in the onset cohort because these were included in 
the definition of T2DM. Please see chapter 4 and chapter 5 for specific list of clinical conditions that were included in onset and "remission" study.

b) Pharmacy: Oral diabetes drug, insulin, statin were identified using administrative pharmacy claims. The indicators were binary fields $(\mathrm{Y} / \mathrm{N})$.

c) Demographic data: Age, gender, state, race were obtained from enrollment data.

d) DCSI score: The DCSI score was calculated using methodology as described previously (98).

\section{Step 6: Data Transformation}

The final data was transformed to create an analytical dataset in a "counting process" input style as previously described $(152,153)$, where each observation represented a Medicare patient's disease condition in a given year (Table 3.4). 
Table 3.4: Sample dataset - counting process input style

\begin{tabular}{|c|c|c|c|c|c|c|c|}
\hline ID & Year & Start & Stop & T2DM & Retinopathy & Neuropathy & $x x x$ \\
\hline 101 & 2008 & 0 & 1 & 1 & $(Y / N)-2008$ & $(Y / N)-2008$ & $(Y / N)-2008$ \\
\hline 101 & 2009 & 1 & 2 & 1 & $(Y / N)-2009$ & $(Y / N)-2009$ & $(Y / N)-2009$ \\
\hline 101 & 2010 & 2 & 3 & 1 & $(Y / N)-2010$ & $(Y / N)-2010$ & $(Y / N)-2010$ \\
\hline 101 & 2011 & 3 & 4 & 1 & $(Y / N)-2011$ & $(Y / N)-2011$ & $(Y / N)-2011$ \\
\hline 101 & 2012 & 4 & 5 & 0 & $(\mathrm{Y} / \mathrm{N})-2012$ & $(Y / N)-2012$ & $(\mathrm{Y} / \mathrm{N})-2012$ \\
\hline 101 & 2013 & 5 & 6 & 0 & $(Y / N)-2013$ & $(Y / N)-2013$ & $(Y / N)-2013$ \\
\hline 101 & 2014 & 6 & 7 & 0 & $(Y / N)-2014$ & $(\mathrm{Y} / \mathrm{N})-2014$ & $(Y / N)-2014$ \\
\hline 101 & 2015 & 7 & 8 & 0 & $(Y / N)-2015$ & $(Y / N)-2015$ & $(Y / N)-2015$ \\
\hline 102 & 2008 & 0 & 1 & 1 & $(Y / N)-2008$ & $(Y / N)-2008$ & $(Y / N)-2008$ \\
\hline 102 & 2009 & 1 & 2 & 1 & $(Y / N)-2009$ & $(Y / N)-2009$ & $(Y / N)-2009$ \\
\hline 102 & 2010 & 2 & 3 & 1 & $(Y / N)-2010$ & $(Y / N)-2010$ & $(\mathrm{Y} / \mathrm{N})-2010$ \\
\hline 102 & 2011 & 0 & 1 & 1 & $(Y / N)-2011$ & $(Y / N)-2011$ & $(Y / N)-2011$ \\
\hline$\cdot$ & $\cdot$ & . & . & $\cdot$ & - & - & $\cdot$ \\
\hline - & . & - & . & . & . & . & . \\
\hline . & . & . & . & . & . & . & - \\
\hline 103 & 2011 & 0 & 1 & 1 & $(Y / N)-2011$ & $(Y / N)-2011$ & $(\mathrm{Y} / \mathrm{N})-2011$ \\
\hline
\end{tabular}


Table 3.5: Topics and example data elements included in the analytical data

\begin{tabular}{lll} 
& Description & Key data elements \\
\hline Clinical & Identified using medical and & Cardiovascular \\
conditions & pharmacy claims & Cerebrovascular \\
& & Peripheral Vascular Disease \\
& & Retinopathy \\
& & Metabolic \\
\hline Pharmacy & $\begin{array}{l}\text { Member's drug usage based on } \\
\text { their pharmacy claims }\end{array}$ & Insulin \\
& & Statin \\
\hline Enrollment & $\begin{array}{l}\text { Coverage with Managed Care } \\
\text { Organization (MCO) }\end{array}$ & Coverage indicators \\
& &
\end{tabular}

These variables were the key independent variables in the analysis (Table 3.5). In the "remission" cohort analysis DCSI score was included. DCSI score was not included in the onset cohort analysis. 


\section{Statistical Analysis}

The data aggregation processes led to the creation of two separate analytical cohorts; a) onset b) "remission." These cohorts were analyzed separately. The aggregated data was analyzed using the following statistical methods;

a. Descriptive statistics: Calculate means, frequencies. Descriptive statistics were used to generate member profile and rates.

b. Survival analysis: Cox proportional hazard models.

Cox proportional hazard models were specified to identify the significant variables associated with T2DM onset and "remission." Statistical significance

was assessed at $\alpha=0.05$. All variables were first included in the unadjusted model. The variables which are significant $(p<0.05)$ were used in an adjusted model.

\section{Presentation of Statistical Results}

All results were reported in aggregated formats only. At no point were the individual or member level information/results reported. Any cells which contained less than 10 individuals were represented as " $<10$." No information is reported at a zip code level or by using specific service dates. This was a requirement from $\mathrm{MCO}$, and helped in providing an additional layer of deidentification. 


\section{Statistical Tools}

Hadoop (Scala, Spark 1.6) and SAS 9.2 were used for data processing and statistical analysis.

\section{Summary of methods}

This study used observational, descriptive, retrospective study using de-identified secondary administrative data. The data was analyzed using SAS and Hadoop and the results were presented in an aggregated format. 
TYPE 2 DIABETES MELLITUS (T2DM) ONSET IN MEDICARE PATIENTS 65 YEARS AND OLDER

\author{
Short running title: T2DM Onset
}

Type 2 Diabetes Mellitus (T2DM) is a chronic condition wherein the beta cells in the body do not produce enough insulin or the body does not use insulin well enough (also called insulin resistance), or there is complete absence of insulin production. T2DM is associated with deleterious macro- and microvascular outcomes (1) and is the seventh leading cause of death in the U.S. affecting 29.1 million people (4). The total estimated national cost of diabetes in the U.S. in 2012 was more than $\$ 245$ billion, up from $\$ 174$ billion in 2007 (5).

T2DM can have a long asymptomatic phase i.e., an individual can sometimes be unaware that they have T2DM for 4 to 7 years before an actual diagnosis (15). The onset (or first occurrence of T2DM) is generally preceded by prediabetes, a condition where an individual's blood sugar is higher than normal, but not high enough to be classified as T2DM (54). Without intervention, pre-diabetes will progress to T2DM onset (55). When left unmanaged, T2DM continues to progress from onset to development of comorbidities and eventually lead to death. T2DM is a progressive disease. Since glucose is a continuous variable, the use of thresholds such as pre-diabetic or T2DM is somewhat arbitrary (64). 
Previous studies have identified several risk factors associated with T2DM onset such as: family history of T2DM; age; obesity; physical inactivity; prior history of gestational diabetes; impaired glucose tolerance; dipocytokines; inflammatory factors and hepatocyte factors; race/ethnicity; alcohol consumption; tobacco smoking; diet $(6-8,10,11)$; high blood pressure (12); appendectomy (13); and hypothyroidism (14). However, there is still additional opportunity to further understand these and other factors that impact the onset of T2DM, especially in the Medicare patients 65 years and older.

Understanding the impact of these comorbidities within the Medicare population is important because the Medicare population differs from the population at large in age, life expectancy, frequency of preexisting comorbid conditions and comorbidities due to progression of T2DM. These differences in turn affect the type of therapeutic and non-therapeutic interventions that are appropriate for this population (154). Understanding these factors will help design better T2DM prevention programs and thereby alleviate disease burden of T2DM and its comorbidities via proactive early intervention. This information will also be useful in cases where T2DM is identified later in its progression (due to late diagnosis) because improved glycemic control is still beneficial and improves quality of life (18).

\section{Research Design and Methods}

\section{Study Design}

This retrospective study used de-identified administrative healthcare claims and enrollment data from a Medicare Advantage Prescription Drug 
(MAPD) health plan offered by a large multistate healthcare company (a managed care organization, MCO). The data was collected during standard course of care. It was not originally collected for the purpose of this study, or any new study on human and animal subjects. Prior to the beginning of the study, this study protocol was reviewed and approved by the Institutional Review Board at University of Louisville. The study was also reviewed by the MCO's privacy and ethics committee.

\section{Study Cohort}

Individuals may qualify for MAPD if they were at least 65 years of age in the year of their Medicare enrollment and met certain additional eligibility criteria. A smaller portion of individuals also qualified for MAPD for reasons such as disability or end stage renal disease (ESRD). Individuals whose initial eligibility criteria for enrollment into MAPD were due to disability or ESRD were excluded from this study.

The large multistate healthcare company insured $>2.4$ million individuals who had continuous MAPD coverage from January to December of 2015. Of these $>2.4$ million individuals, there were more than 1.7 million individuals who had "age $\geq 65$ " as the original reason for Medicare entitlement. The rest qualified under Medicare disability or ESRD eligibility provisions and were excluded from this study. Of these 1.7 million individuals, there were 265,554 members who had continuous MAPD coverage with the MCO from January 2008 to December 2015. Starting with the sampling frame of these 265,554 individuals, this study excluded the following during 2008 and 2015: a) individuals who had bariatric 
surgery (including gastric bypass, laproscopic gastroenterostomy) $(n=619)$; b) individuals with polycystic ovary syndrome $(n=131)$ and lipodystrophy $(n=718)$ were excluded because these individuals may have medications which have competing indications with T2DM; c) individuals with any diagnostic codes related to nephropathy were excluded because a previous study published by Kaiser Permanente indicated that worsening renal function could lead to improved glycemic control (20). In the present study, chronic kidney disease (CKD) was excluded $(n=109,243)$ in order to identify a population without irreversible end organ damage. In the current study we excluded pre-existing nephropathy $(41.3 \%)$ of the study sample. Previous research suggested the rate of CKD among individuals 65 years and older was $61 \%$ in a year. The CKD rate in the current study was likely lower because individuals with "disability" and "ESRD" as their initial reason for Medicare enrollment were excluded from the sampling frame (155); d) individuals who had drug and/or steroid induced diabetes were excluded because the pathway for T2DM onset in these individuals may be different from normal pathways for T2DM onset $(n=3,436)$; e) individuals with type 1 diabetes (determined by ICD codes) were excluded because type 1 diabetes has a different etiology from T2DM $(n=25,535) ; f)$ individuals who had T2DM related diagnostics codes only during inpatient hospital stay and do not have T2DM diagnostic claims or T2DM related pharmacy claims in a non-hospital setting were excluded to account for potential surgery induced dysglycemia ( $n=1,772)$; and, g) certain restricted groups as 
required by the MCO's privacy and legal agreements were also excluded from this study. The MCO's IRB required this exclusion $(n=2,238)$.

\section{Inclusions}

To be included in T2DM onset cohort individuals were required to have no T2DM related claims in years 2008, 2009, 2010 and 2011 and have one of the following qualifiers: a) individuals who had no T2DM related claims in years 2012, 2013, 2014 and 2015; or b) individuals who had T2DM onset in any year between 2012 and 2015. Upon onset, individuals were also required to have T2DM related claims every year until the end of the study (Appendix 4.1).

After the above mentioned inclusion and exclusion criteria, the final T2DM onset cohort included 89,390 Medicare patients. 4,314 individuals had T2DM

onset (T2DM onset group) and 85,076 individuals had no T2DM onset (no T2DM onset group) during the study period (Appendix 4.1). The baseline year was 2011, since the entire study cohort had no T2DM in 2008, 2009, 2010 and 2011.

\section{Case definitions}

T2DM: T2DM individuals were identified using administrative medical and pharmacy claims data using the following criteria: a) at least two diagnoses of T2DM (International Classification of Diseases, Ninth Revision, Clinical Modification [ICD-9-CM] and/or (International Classification of Diseases, Tenth Revision, Clinical Modification [ICD-10-CM]; b) at least two pharmacy scripts related to T2DM; and, c) at least 2 claims (medical or pharmacy) related to T2DM. A minimum of two claims were used in the identification of T2DM as a 
means of excluding patients who were potentially miscoded and patients who were suspected to have T2DM but were never formally diagnosed.

Covariates: T2DM co-morbidities were defined using administrative claims data as described previously using both ICD-9 and ICD-10 diagnostic codes (98). The following co-morbidities were included in the study: a) retinopathy: other retinopathy, retinal edema, cystoid macular edema/degeneration (CSME), other retinal disorders, retinal detachment and blindness; b) neuropathy: amyotrophy, cranial nerve palsy, neurogenic bladder, gastroparesis/diarrhea and orthostatic hypotension; c) cerebrovascular: transient ischemic attack, stroke; d) cardiovascular diseases: atherosclerosis, other ischemic heart disease (IHD), angina pectoris, other chronic IHD, myocardial infarction, ventricular fibrillation and arrest, atrial fibrillation, arrest, other atherosclerotic cardiovascular disease, old myocardial infarction, heart failure, atherosclerosis severe, and aortic aneurysm/dissection; e) peripheral vascular disease (PVD): other aneurysm of lower extremities, foot wound and complications, embolism/thrombosis in lower extremities, ulcer of lower limbs; f) hypertension; and g) hyperlipidemia.

Demographic information (age, gender, and race) was based on enrollment data. T2DM related pharmacy and statin use were determined using administrative pharmacy data.

\section{Statistical Analysis}

Data aggregation and the implementation of inclusions and exclusions was done in Hadoop (using Spark, Scala and Hive). Hadoop is an open source 
software framework for distributed processing and distributed storage of very large datasets (151). The processing was done in Hadoop because of the volume of the data which included $\geq 2.4$ million individuals' medical and pharmacy claims data from 2008 to 2015 . Means and frequencies to generate member profiles was calculated using Hadoop. The analytical dataset was created in a counting process input style $(152,153)$. This data was then moved into SAS 9.2. (SAS Institute Inc., Cary NC) to calculate rate of T2DM onset. Cox proportional hazard models were specified to identify the significant variables associated with T2DM onset. SAS procedure PROC PHREG was used for Cox proportional hazard models (152).

The unadjusted model was developed using demographic, pharmacy, and clinical conditions. The level of significance for unadjusted models was $p<0.05$. The model was developed using Cox proportional hazard. Variables that were significant in the unadjusted model were then included in the adjusted model. In the final analysis, the adjusted model included the demographic variables (gender and race), pharmacy (statin use) and clinical conditions (hypertension, hyperlipidemia, heart failure, ulcer of lower limbs, atherosclerosis, retinal edema, gastroparesis, orthostatic hypotension, cerebrovascular, atherosclerosis, other ischemic heart disease (IHD), other chronic IHD, myocardial infarction (MI), ventricular fibrillation and arrest, other atherosclerotic cardiovascular disease (ASCVD), old MI, atherosclerosis severe, embolism, blindness and low vision, other retinopathy and angina pectoris ). The level of significance for all statistical tests was $p<0.05$. 


\section{Results}

There were 89,390 Medicare patients 65 years and older who did not have T2DM in 2008, 2009, 2010 and 2011. Of these, 4,314 Medicare patients were diagnosed with T2DM between 2012 and 2015, while the remaining 85,076 Medicare patients continued to have no T2DM diagnosis until the end of study, i.e., 2015. The cumulative rate of diagnosed T2DM onset in the study cohort was $4.82 \%(4314 / 89,390)($ Table 4.1$)$. The rates of diagnosed T2DM were $0.82 \%$, $0.88 \%, 1.04 \%$ and $2.09 \%$ in years $2012,2013,2014$ and 2015 , respectively.

In the baseline year (2011), the average age of Medicare patients in the T2DM onset cohort $(n=4,314)$ was 72.1 years compared to the average age of 73.0 years for the no-onset cohort $(n=85,076)(p<0.0001)$. The T2DM onset cohort consisted of $60.3 \%$ females compared to $63.5 \%$ females in the no-onset cohort (Table 4.1) $(p<0.0001)$. Even though the difference in the gender $(3.2 \%)$ and age (1.9 years) are statistically significant, these groups are not very significant from each other from a theoretical perspective (Table 4.1).

At baseline there were significantly more non-white (African American, Hispanic) individuals (19.5\%) in the T2DM onset cohort compared to the T2DM no onset cohort $(11.7 \%)(p<0.0001)($ Table 4.1$)$.

At baseline, the T2DM onset cohort had significantly higher prevalence of disease conditions compared to no-onset cohort. Individuals in the T2DM onset cohort had higher prevalence of clinical conditions such as hypertension $(70.1 \%$ vs. $59.1 \%$ ), hyperlipidemia ( $69.4 \%$ vs. $59.5 \%$ ), cardiovascular (atherosclerosis ( $5.0 \%$ vs. $7.2 \%)$, other ischemic heart disease ( $0.8 \%$ vs $1.5 \%)$, angina pectoris 
(2.1\% vs. $3.6 \%)$, other chronic ischemic heart disease (12.3\% vs. $15.4 \%)$, other atherosclerotic cardiovascular disease ( $0.9 \%$ vs. $1.3 \%)$, history of myocardial infarction (3.2\% vs. $4.6 \%)$, heart failure (3.1\% vs. $4.8 \%)),(p<0.05)($ Table 4.2$)$. Even though the clinical conditions related to cardio vascular disease were significant, they might not be a clinically significant difference (Table 4.2).

In bivariate and multivariate analysis, male gender, non-white (African American, Hispanic), statin use, hypertension, hyperlipidemia, heart failure, ulcer of lower limbs, atherosclerosis, other retinopathy, angina pectoris, blindness and low vision, absence of other ischemic heart disease (IHD) was associated with T2DM onset in Medicare patients $(p<0.05)$ (Figure 4.1, Figure 4.2 and Appendix $4.2)$.

\section{Conclusions}

The four year rate of T2DM onset was 48.2 per 1000 population. The average rate of T2DM onset was 12.05 per 1000 per year. This rate was consistent with the unadjusted incidence of T2DM of 11.5 per 1000 in the U.S. 65 years or older population (4).

Previously published research has documented some of the risk factors for T2DM on: family history of T2DM, age, obesity, and physical inactivity, prior history of gestational diabetes race, alcohol consumption, tobacco smoking, diet $(6-8,10,11)$. Some of the known risk factors that characterize metabolic syndrome, a precursor for T2DM: impaired glucose tolerance; dipocytokines; inflammatory factors and hepatocyte factors; and hypertension $(12,156)$. 
Gender and Race: The study presented here found that males, non-white (African American, Hispanic) are significantly associated with T2DM onset in the Medicare patients. Male gender was more likely to be associated with T2DM onset compared to female gender $(\mathrm{p}<0.0001)$. Previous studies found globally that males are more likely to develop T2DM than females $(157,158)$. This trend has changed over the last 50 years. Females were more likely to have diagnosed T2DM, but in recent years, the increasing sedentary lifestyles and diets of males appears to be associated with increased prevalence of T2DM $(159,160)$. Consistent with prior studies, the current investigation found that African Americans and Hispanics were more likely to have an onset of T2DM than Caucasians in the Medicare population (161). These findings further emphasize the need for culturally sensitive interventions to help ameliorate racial disparities of T2DM onset especially in the elderly, sedentary population.

Statins: This study also evaluated the role of statin use on T2DM onset. The HMG-CoA inhibitors (statins), are one of the most commonly prescribed drugs worldwide to reduce cholesterol, and prevent or manage coronary heart disease (162). In a meta-analysis of 13 randomized trials, the use of statins was associated with a $9 \%$ increase in the risk of T2DM onset, with the risk being the highest in the older population. However, considering its effect on reducing coronary events, previous studies have recommended no change in clinical decision making for medium to high risk of cardiovascular diseases (163). Similar to prior studies, the present investigation found that statin use was associated with the onset of T2DM in Medicare patients $(p<0.0001)$ and recommend 
surveillance of dysglycaemia among non-T2DM Medicare patients who are receiving statin treatment.

Hypertension: High blood pressure affects approximately a billion people worldwide and is a common yet modifiable risk factor for T2DM onset. Individuals who have hypertension are 2.5 times more likely to develop T2DM $(164,165)$. In the present study, hypertension is associated with T2DM onset $(p<0.05)$.

Retinopathy: Prior investigations suggest that nearly $21 \%$ of individuals who are diagnosed with T2DM also have some level of retinopathy at the time of diagnosis (166). In individuals who do not have T2DM related retinopathy, the symptoms are generally a manifestation of either retinal micro aneurysms or blot hemorrhages. Advanced age and hypertension are associated with the development of non-diabetic retinopathy but hypertension and T2DM are risk factors for retinopathy in individuals who are $<65$ years of age years (167).

The present study found that retinopathy (other retinopathy, blindness or low vision) was associated with T2DM onset in Medicare patients $(p<0.05)$. One possible explanation is that when an optometrist or other provider evaluates a Medicare individual for non-diabetic retinopathy, they might recommend T2DM screening. This interaction with their provider and subsequent evaluation of blood glucose can lead to diagnosis of T2DM.

Hyperlipidemia: Hyperlipidemia is key component of metabolic syndrome. Metabolic syndrome refers to the co-occurrence of several known cardiovascular risk factors such as atherogenic dyslipidemia (elevated triglycerides and low 
high-density lipoprotein [HDL]), high blood pressures, elevated glucose levels, and abdominal obesity with pro-thrombotic and pro-inflammatory states (168). These conditions have a common pathway and are interrelated. Individuals with metabolic syndrome are at a significantly higher risk of developing T2DM (169). In the study presented here, hyperlipidemia was associated with T2DM onset in the Medicare patients $(p<0.05)$. Hyperlipidemia and hypertension are significant predictors of T2DM onset, and may be a proxy for metabolic syndrome as a predictor for T2DM onset (170).

Peripheral vascular disease (PVD): PVD is characterized by vascular smooth muscle cell dysfunction, endothelial dysfunction, inflammation and hypercoagulability. PVD is a frequent comorbidity in T2DM. Vascular ulcers such as ulcers of lower limbs are a common cause for older patients to visit a provider (171). In the study presented here non-diabetic ulcer of lower limbs was associated with T2DM onset in Medicare patients $(p<0.05)$.

Cardiovascular disease (CVD): As previously mentioned above, metabolic syndrome is characterized by clustering of risk factors such as prothrombotic, hypertension and dyslipidemia. Metabolic syndrome independently contributes to CVD and T2DM (172). This study evaluated several cardiovascular conditions associated with T2DM: angina pectoris, atherosclerosis, heart failure, other chronic IHD. Clinical and lab data related to metabolic syndrome was not available, to further evaluate if the observed associations are due to the underlying metabolic syndrome. Despite this limitation, these finding can still provide valuable information to providers about the prognosis of the T2DM. 
Some of the cardiovascular conditions that were significantly associated with T2DM were: a) angina pectoris: angina pectoris (angina) is characterized by chest pain or discomfort, often associated with myocardial ischemia, arterial insufficiency or hypoxia. Angina is generally a symptom for an underlying cause such as coronary artery disease (173). Angina pectoris is associated with T2DM onset in the present study $(p<0.05)$. Associations found with angina and T2DM onset must be re-evaluated by looking at the underlying causes; b) other chronic IHD: this diagnostic code is associated with loss of elasticity and thickening of the coronary arteries, leading to progressive arterial insufficiency. This study found that in bivariate analysis other chronic IHD was associated with T2DM onset. However when adjusted for other factors IHD had a protective effect on T2DM onset. This finding suggests that individuals who have hypertension and hyperlipidemia and also have IHD might have different behavior towards their health and hence might be able to delay onset of T2DM (174).; c) heart failure and atherosclerosis were associated with T2DM onset in Medicare patients.

In summary, male gender, non-white ethnicity (African American, Hispanics), statin use, hypertension, hyperlipidemia, heart failure, ulcer of lower limbs, atherosclerosis, other retinopathy, angina pectoris, blindness and low vision, absence of other chronic ischemic heart disease (IHD) was associated with T2DM onset in Medicare patients. African American and Hispanics (without regard to gender), statins, hyperlipidemia, and hypertension are findings in agreement with previously reported risk factors with development of T2DM. The new associations with T2DM discovered in this investigation include: heart 
failure, ulcer of lower limbs, atherosclerosis, other retinopathy, angina pectoris, blindness and low vision, and absence of other chronic ischemic heart disease (IHD).

Note: The tables and figures are placed at the end of the article as per the requirement of the journal. 


\section{Tables and Figure Legends}

Table 4.1: Baseline demographic and T2DM onset characteristics

\begin{tabular}{lccc} 
Characteristic & $\begin{array}{c}\text { No Onset* } \\
(\mathbf{n = 8 5 , 0 7 6 )}\end{array}$ & $\begin{array}{c}\text { Onset } \\
(\mathbf{n}=\mathbf{4}, \mathbf{3 1 4})\end{array}$ & P value \\
\hline Age, mean (SD), years & $73.0(5.6)$ & $72.1(5.1)$ & $<.0001$ \\
Female sex & $63.5 \%$ & $60.3 \%$ & $<.0001$ \\
Race/ethnicity & & & $<.0001$ \\
White & $75,051(88.3)$ & $3,465(80.5)$ & \\
Black & $6,316(7.4)$ & $542(12.5)$ & \\
Others & $3,640(4.3)$ & $300(7.0)$ & \\
\hline
\end{tabular}

Data are represented as $\mathrm{n}(\%)$.

${ }^{*}$ Missing data in no onset cohort: race $(n=69)$.

${ }^{* *}$ Missing data in onset cohort: race $(n=7)$. 
Table 4.2: Baseline comorbidities

\begin{tabular}{|c|c|c|c|}
\hline Characteristic & $\begin{array}{c}\text { No Onset } \\
(n=85,076)\end{array}$ & $\begin{array}{c}\text { Onset } \\
(n=4,314)\end{array}$ & $P$ value \\
\hline Hypertension & $50,336(59.2)$ & $3,024(70.1)$ & $<.0001$ \\
\hline Hyperlipidemia & $50,636(59.5)$ & $2,993(69.4)$ & $<.0001$ \\
\hline \multicolumn{4}{|l|}{ Retinopathy } \\
\hline Other retinopathy & $795(0.9)$ & $42(1.0)$ & 0.7950 \\
\hline Retinal edema & $351(0.4)$ & $15(0.3)$ & 0.5150 \\
\hline CSME & $430(0.5)$ & $26(0.6)$ & 0.3820 \\
\hline Other retinal disorders & $294(0.3)$ & $10(0.2)$ & 0.2100 \\
\hline Retinal detachment & $647(0.8)$ & $37(0.9)$ & 0.4750 \\
\hline Blindness & $535(0.6)$ & $32(0.7)$ & 0.3620 \\
\hline \multicolumn{4}{|l|}{ Neuropathy } \\
\hline Neurogenic bladder & $154(0.2)$ & $<10$ & 0.7770 \\
\hline Gastroparesis/diarrhea & $174(0.2)$ & $12(0.3)$ & 0.3000 \\
\hline Orthostatic hypotension & $1,226(1.4)$ & $57(1.3)$ & 0.5190 \\
\hline \multicolumn{4}{|l|}{ Cerebrovascular } \\
\hline TIA & $1,486(1.7)$ & $78(1.8)$ & 0.7640 \\
\hline Stroke & $5,104(6.0)$ & $288(6.7)$ & 0.0690 \\
\hline \multicolumn{4}{|l|}{ Cardiovascular } \\
\hline Atherosclerosis & $4,216(5.0)$ & $311(7.2)$ & $<.0001$ \\
\hline Other IHD & $675(0.8)$ & $66(1.5)$ & $<.0001$ \\
\hline Angina pectoris & $1,773(2.1)$ & $155(3.6)$ & $<.0001$ \\
\hline Other chronic IHD & $10,445(12.3)$ & $664(15.4)$ & $<.0001$ \\
\hline Myocardial infarction & $588(0.7)$ & $37(0.9)$ & 0.2000 \\
\hline Ventricular fibrillation, arrest & $5,508(6.5)$ & $308(7.1)$ & 0.0840 \\
\hline Atrial fibrillation, arrest & $78(0.1)$ & $<10$ & 0.3220 \\
\hline Other ASCVD & $761(0.9)$ & $55(1.3)$ & 0.0100 \\
\hline Old myocardial infarction & 2,761 (3.2) & $197(4.6)$ & $<.0001$ \\
\hline Heart failure & $2,673(3.1)$ & $208(4.8)$ & $<.0001$ \\
\hline Atherosclerosis, severe & $38(0.0)$ & $<10$ & 0.9590 \\
\hline Aortic aneurysm/dissection & $1,307(1.5)$ & $52(1.2)$ & 0.0830 \\
\hline \multicolumn{4}{|l|}{ Peripheral vascular disease } \\
\hline Other aneurysm, LE & $35(0.0)$ & $<10$ & 0.3770 \\
\hline Foot wound + complication & $26(0.0)$ & $<10$ & 0.7860 \\
\hline Embolism/thrombosis (LE) & $75(0.1)$ & $<10$ & 0.5520 \\
\hline Ulcer of lower limbs & $493(0.6)$ & $22(0.5)$ & 0.5560 \\
\hline
\end{tabular}

Data are expressed as $n(\%)$. Baseline year $=2011$.

IHD, ischemic heart disease; LE, lower extremity; TIA, transient ischemic attack; ASCVD, atherosclerotic cardiovascular disease 
Figure 4.1: Unadjusted Cox proportional hazard for T2DM onset

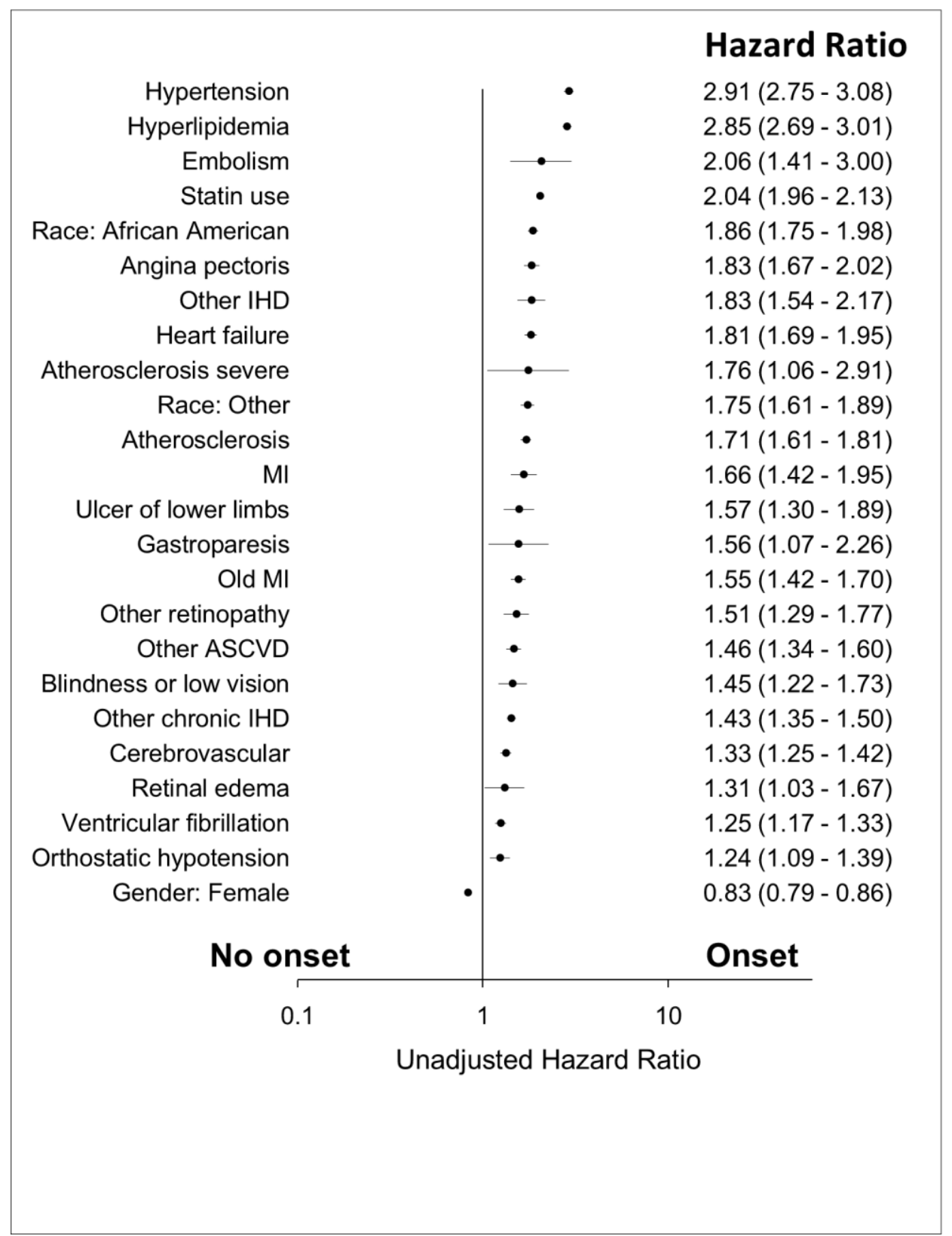

MI, Myocardial infarction; ASCVD, atherosclerotic cardiovascular disease; IHD, ischemic heart disease; Race other, non-white and non-African American. 
Figure 4.2: Adjusted Cox proportional hazard for T2DM onset

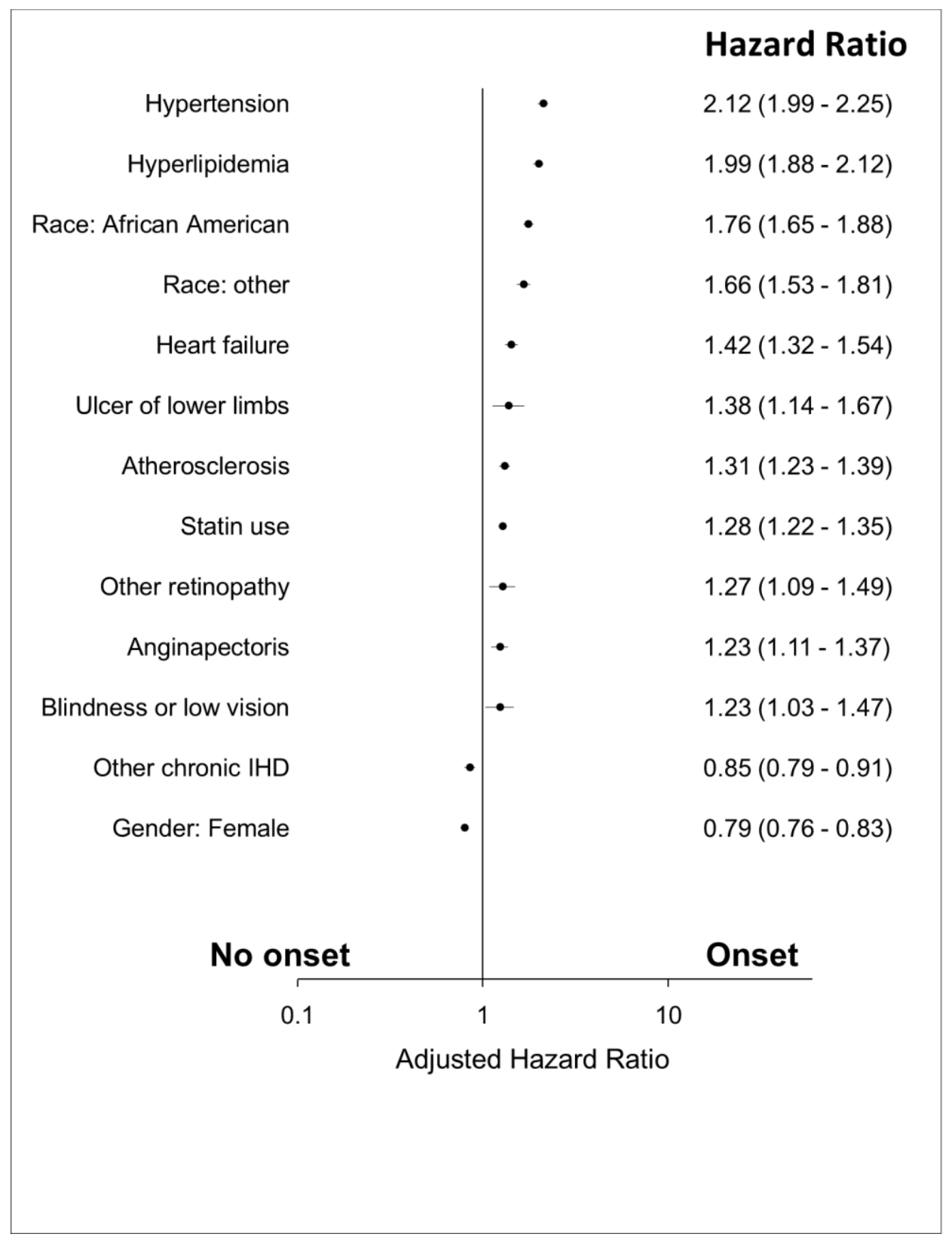

IHD, ischemic heart disease; Race other, non-white and non-African American. 
Appendix 4.1: T2DM Onset

T2DM Diagnosis

\begin{tabular}{|c|c|c|c|c|c|c|c|c|c|}
\hline $2008^{\S}$ & 2009 & 2010 & 2011 & 2012 & 2013 & 2014 & 2015 & PATTERN\$§ & Description \\
\hline $\mathrm{NO}$ & $\mathrm{NO}$ & $\mathrm{NO}$ & NO & $\mathrm{NO}$ & NO & NO & $\mathrm{NO}$ & 00000000 & $\begin{array}{c}\text { No onset } \\
(\mathrm{n}=85,076)\end{array}$ \\
\hline $\begin{array}{l}\text { NO } \\
\text { NO }\end{array}$ & $\begin{array}{l}\text { NO } \\
\text { NO }\end{array}$ & $\begin{array}{l}\text { NO } \\
\text { NO }\end{array}$ & $\begin{array}{l}\text { NO } \\
\text { NO }\end{array}$ & $\begin{array}{l}\text { NO } \\
\text { NO }\end{array}$ & $\begin{array}{l}\text { NO } \\
\text { NO }\end{array}$ & $\begin{array}{l}\text { NO } \\
\text { YES }\end{array}$ & $\begin{array}{l}\text { YES } \\
\text { YES }\end{array}$ & $\begin{array}{l}00000001 \\
00000011\end{array}$ & \multirow{3}{*}{$\begin{array}{c}\text { Onset } \\
(n=4,314)\end{array}$} \\
\hline NO & NO & NO & NO & NO & YES & YES & YES & 00000111 & \\
\hline $\mathrm{NO}$ & $\mathrm{NO}$ & $\mathrm{NO}$ & NO & YES & YES & YES & YES & 00001111 & \\
\hline NO & NO & NO & YES & YES & YES & YES & YES & 00011111 & \multirow{3}{*}{$\begin{array}{c}\text { Onset } \\
\text { (excluded) }\end{array}$} \\
\hline NO & NO & YES & YES & YES & YES & YES & YES & 00111111 & \\
\hline $\mathrm{NO}$ & YES & YES & YES & YES & YES & YES & YES & 01111111 & \\
\hline $\mathrm{NO}$ & $\mathrm{NO}$ & $\mathrm{NO}$ & NO & $\mathrm{NO}$ & NO & YES & $\mathrm{NO}$ & 00000010 & \multirow{2}{*}{$\begin{array}{l}\text { No continuous onset } \\
\text { (excluded) }\end{array}$} \\
\hline NO & $\mathrm{NO}$ & NO & NO & $\mathrm{NO}$ & YES & NO & YES & 00000101 & \\
\hline
\end{tabular}

$\S \mathrm{NO}=$ no type 2 diabetes diagnosis, and $\mathrm{YES}$ = type 2 diabetes diagnosis

$\S \S 0=$ no T2DM and $1=$ presence of T2DM 
Appendix 4.2: Unadjusted and adjusted Cox proportional hazard for T2DM onset

\begin{tabular}{|c|c|c|c|c|c|}
\hline \multirow[b]{3}{*}{ Characteristic } & \multicolumn{4}{|c|}{ Any onset $(n=4,314)$} & \multirow[b]{3}{*}{$P$ value } \\
\hline & \multicolumn{4}{|c|}{ Hazard Ratio $(95 \% \mathrm{Cl})$} & \\
\hline & $n(\%)$ & Unadjusted & $P$ Value & Fully adjusted & \\
\hline \multicolumn{6}{|l|}{ Sex } \\
\hline Men & $1,711(5.2)$ & $1.0(\mathrm{ref})$ & $<0.0001$ & 1.0 (ref) & $<0.0001$ \\
\hline Women & $2,603(4.6)$ & $0.83(0.79-0.86)$ & & $0.79(0.76-0.83)$ & \\
\hline \multicolumn{6}{|l|}{ Race } \\
\hline White & $3,465(4.4)$ & $1.0(\mathrm{ref})$ & $<0.0001$ & $1.0(\mathrm{ref})$ & $<0.0001$ \\
\hline African American & $542(7.9)$ & $1.86(1.75-1.98)$ & & $1.76(1.65-1.88)$ & \\
\hline Other & $300(7.6)$ & $1.75(1.61-1.89)$ & & $1.66(1.53-1.81)$ & \\
\hline \multicolumn{6}{|l|}{ Statins } \\
\hline No statins & $2,228(4.2)$ & $1.0(\mathrm{ref})$ & $<0.0001$ & 1.0 (ref) & $<0.0001$ \\
\hline Statins & $2,086(5.7)$ & $2.04(1.96-2.13)$ & & $1.28(1.22-1.35)$ & \\
\hline \multicolumn{6}{|l|}{ Clinical Conditions } \\
\hline No hypertension & $1,290(3.6)$ & $1.0(\mathrm{ref})$ & $<0.0001$ & $1.0(\mathrm{ref})$ & $<0.0001$ \\
\hline Hypertension & $3,024(5.7)$ & $2.91(2.75-3.08)$ & & $2.12(1.99-2.25)$ & \\
\hline No hyperlipidemia & $1,321(3.7)$ & $1.0(\mathrm{ref})$ & $<0.0001$ & $1.0(\mathrm{ref})$ & $<0.0001$ \\
\hline Hyperlipidemia & $2,993(5.6)$ & $2.85(2.69-3.01)$ & & $1.99(1.88-2.12)$ & \\
\hline \multicolumn{6}{|l|}{ Retinopathy } \\
\hline No other retinopathy & $4,272(4.8)$ & $1.0(\mathrm{ref})$ & $<0.0001$ & 1.0 (ref) & 0.0024 \\
\hline Other retinopathy & $42(5.0)$ & $1.51(1.29-1.77)$ & & $1.27(1.09-1.49)$ & \\
\hline No retinal edema & $4,299(4.8)$ & 1.0 (ref) & 0.0303 & $1.0(\mathrm{ref})$ & 0.1604 \\
\hline Retinal edema & $15(4.1)$ & $1.31(1.03-1.67)$ & & $1.19(0.93-1.53)$ & \\
\hline $\begin{array}{l}\text { No blindness or low } \\
\text { vision }\end{array}$ & $4,282(4.8)$ & $1.0(\mathrm{ref})$ & $<0.0001$ & $1.0(\mathrm{ref})$ & 0.0194 \\
\hline
\end{tabular}




\begin{tabular}{|c|c|c|c|c|c|}
\hline \multirow[b]{3}{*}{ Characteristic } & \multicolumn{4}{|c|}{ Any onset $(n=4,314)$} & \multirow[b]{3}{*}{$P$ value } \\
\hline & \multicolumn{4}{|c|}{ Hazard Ratio $(95 \% \mathrm{Cl})$} & \\
\hline & n (\%) & Unadjusted & P Value & Fully adjusted & \\
\hline Blindness or low vision & $32(5.6)$ & $1.45(1.22-1.73)$ & & $1.23(1.03-1.47)$ & \\
\hline \multicolumn{6}{|l|}{ Neuropathy } \\
\hline No gastroparesis & $4,302(4.8)$ & 1.0 (ref) & 0.0193 & 1.0 (ref) & 0.0641 \\
\hline $\begin{array}{l}\text { Gastroparesis } \\
\text { No orthostatic }\end{array}$ & $12(6.5)$ & $1.56(1.07-2.26)$ & & $1.42(0.98-2.06)$ & \\
\hline hypotension & $4,257(4.8)$ & $1.0(\mathrm{ref})$ & 0.0007 & 1.0 (ref) & 0.8693 \\
\hline Orthostatic hypotension & $57(4.4)$ & $1.24(1.09-1.39)$ & & $0.99(0.87-1.12)$ & \\
\hline No cerebrovascular & $3,992(4.8)$ & 1.0 (ref) & $<0.0001$ & 1.0 (ref) & 0.7160 \\
\hline $\begin{array}{l}\text { Cerebrovascular } \\
\text { Cardiovascular }\end{array}$ & \multicolumn{4}{|c|}{ Cardiovascular } & \\
\hline No atherosclerosis & $4,003(4.7)$ & $1.0(\mathrm{ref})$ & $<0.0001$ & 1.0 (ref) & $<.0001$ \\
\hline Atherosclerosis & $311(6.9)$ & $1.71(1.61-1.81)$ & & $1.31(1.23-1.39)$ & \\
\hline No other IHD & 4,248 (4.8) & 1.0 (ref) & $<0.0001$ & 1.0 (ref) & 0.1124 \\
\hline Other IHD & $66(8.9)$ & $1.83(1.54-2.17)$ & & $1.16(0.97-1.39)$ & \\
\hline No angina pectoris & $4,159(4.8)$ & 1.0 (ref) & $<0.0001$ & 1.0 (ref) & $<.0001$ \\
\hline $\begin{array}{l}\text { Angina pectoris } \\
\text { No other chronic }\end{array}$ & $155(8.0)$ & $1.83(1.67-2.02)$ & & $1.23(1.11-1.37)$ & \\
\hline IHD & $3,650(4.7)$ & 1.0 (ref) & $<0.0001$ & 1.0 (ref) & $<.0001$ \\
\hline $\begin{array}{l}\text { Other chronic IHD } \\
\text { No myocardial }\end{array}$ & $664(6.0)$ & $1.43(1.35-1.50)$ & & $0.85(0.79-0.91)$ & \\
\hline $\begin{array}{l}\text { infarction } \\
\text { Myocardial }\end{array}$ & $4,277(4.8)$ & 1.0 (ref) & $<0.0001$ & 1.0 (ref) & 0.2523 \\
\hline $\begin{array}{l}\text { infarction } \\
\text { No ventricular }\end{array}$ & $37(5.9)$ & $1.66(1.42-1.95)$ & & $1.10(0.93-1.30)$ & \\
\hline fibrillation, arrest & $4,006(4.8)$ & 1.0 (ref) & $<0.0001$ & 1.0 (ref) & 0.3138 \\
\hline
\end{tabular}




\section{Any onset $(\mathrm{n}=4,314)$}

Hazard Ratio (95\% Cl)

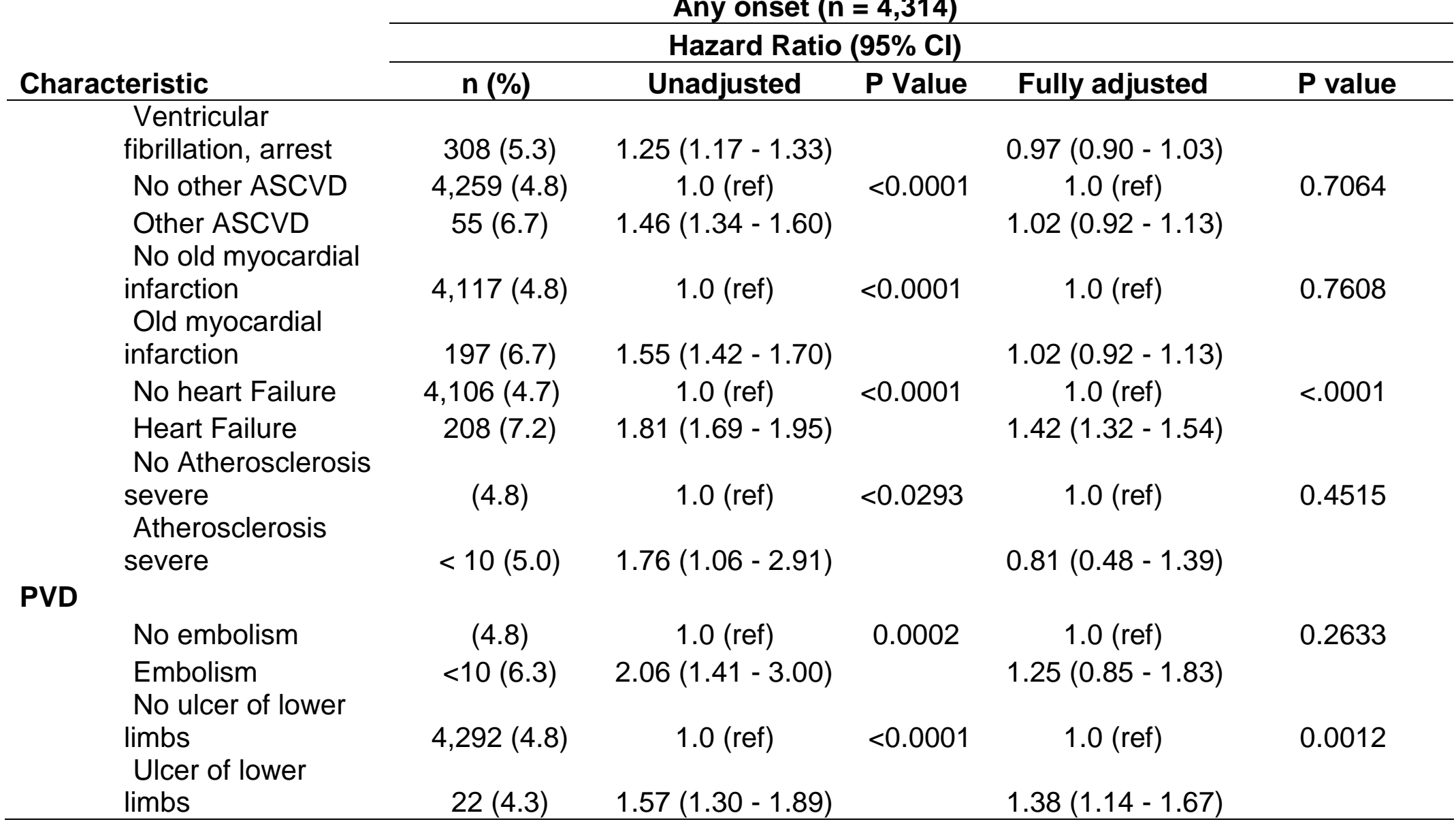

Fully adjusted model includes all variables in this table. Data represented as $\mathrm{n}(\%)$ for baseline year (2011)

${ }^{*}$ Missing data in No Onset cohort: race $(n=69) .{ }^{* *}$ Missing data in Onset cohort: race $(n=7)$. 
TYPE 2 DIABETES MELLITUS (T2DM) “REMISSION" IN MEDICARE PATIENTS 65 YEARS AND OLDER WITHOUT BARIATRIC SURGERY SHORT RUNNING TITLE: T2DM "REMISSION"

Type 2 diabetes mellitus (T2DM) is generally considered as a chronic, incurable, progressive and life-long disease condition. T2DM disease management focuses primarily on glycemic control and management of the macro- and microvascular complications that may be associated with hyperglycemia $(175,176)$. However, a growing body of evidence indicates that T2DM remission can be achieved in certain individuals (107).

The American Diabetes Association (ADA) has defined the term remission as "...achieving glycaemia below the diabetic range in the absence of active pharmacologic or surgical therapy." ADA classifies remission into one of the following three types: a) partial remission, defined as sub-diabetic hyperglycemia without active pharmacologic therapy or ongoing procedures for at least 1 year. Sub-diabetic hyperglycemia is characterized by $\mathrm{HbA} 1_{c}<6.5 \%$ and/or fasting glucose 100-125 mg/dL [5.6-6.9 mmol/l]; b) complete remission, defined as "normal" measures of glucose metabolism without active pharmacologic therapy or ongoing procedures for at least 1 year. Normal measure of glucose metabolism is when $\mathrm{HbA} 1 \mathrm{c}$ is in the normal range, $<5.7 \%,<39 \mathrm{mmol} / \mathrm{mol}$, fasting 
glucose $<100 \mathrm{mg} / \mathrm{dL}$ [5.6 mmol/l]); and c) prolonged remission, defined as complete remission for at least 5 years (106).

Current evidence on T2DM remission suggests that there are at least two key approaches for T2DM remission namely, metabolic/bariatric surgery and/or intensive lifestyle management (175). Of these two approaches, bariatric ("weight-loss") surgery has been proved to be effective in treating T2DM patients whose BMI is $35 \mathrm{~kg} / \mathrm{m} 2$ or higher (130) with a success of achieving T2DM remission between $24 \%$ and $95 \%(131,132)$. Bariatric surgery is considered relatively safe with 30 day mortality between $0.1 \%$ and $1.1 \%$. However, the risk of developing acute complications and long term complications is high (17\%). The rate of reoperation following bariatric surgery is around $7 \%$ and patients are often required to have a close postoperative medical monitoring (177-180).

Current understanding of T2DM remission among non-bariatric patients intervention is very limited. A recent post hoc randomized controlled study from the Look AHEAD (Action for Health for Diabetes) and the 2014 Kaiser Permanente study have shown metabolic/bariatric surgery is not the only pathway to remission. Although rare, T2DM remission does occur in certain populations without bariatric surgery $(19,20,107)$. However, very little is known about remission and factors associated with it.

Understanding factors predictive of remission within the Medicare patients 65 years and older is important because Medicare population differs from population at large by their age, life expectancy, and frequency of comorbidities (154), and have a significantly higher prevalence of T2DM compared to the population at 
large (181). Therefore, understanding these factors have substantial clinical relevance to the design of interventions which lead to remission. In addition, these data could be used to help design better T2DM management programs.

In order to identify remission as per the ADA definition, a researcher would need both pharmacy and laboratory data $\left(\mathrm{HbA} 1_{\mathrm{c}}\right)$. However, laboratory test data are not always available to researchers, especially to those researchers who only rely on administrative claims data for their analytical work (99). Therefore, a modified definition for remission is offered in the present study. In this investigation, the modified definition of remission is enclosed in quotation marks (as "remission").

In summary, this study was conducted to estimate the rate of type 2 diabetes mellitus (T2DM) "remission" and to identify the factors that are associated with T2DM "remission" in Medicare patients 65 years and older who did not undergo bariatric surgery using administrative healthcare claims data in the absence of $\mathrm{HbA1}$ c data.

\section{Research Design and Methods}

\section{Study Design}

This study was a retrospective cohort analysis using Medicare Advantage Prescription Drug (MAPD) health plan data. This administrative data includes member level enrollment, medical claims and pharmacy claims data. These data were collected during the standard course of care. It was not originally collected for the purpose of research or any new study of human or animal subjects. Prior 
to the start of the study this study protocol was reviewed and approved by the University of Louisville Institutional Review Board. The study was also approved by the MCO's review board.

\section{Study Cohort}

The federal government offers original Medicare insurance, which includes Medicare Part A (Hospital Insurance) and Medicare Part B (Medical Insurance). Part C (or Medicare advantage) are offered through private insurance companies. Part C includes the benefits of Part A, Part B and sometimes the optional Part D (prescription drug coverage) along with several choices of programs and services specific to each Managed Care Organization (MCO). Individuals can only be enrolled in Part C if they qualify for Medicare part A and part B. An individual may qualify for Medicare if they are at least 65 years old in the year of their Medicare enrollment and meet certain additional eligibility criteria. In addition, some individuals may qualify for Medicare for other reasons such as disabilities or end stage renal disease (ESRD). Individuals whose initial reason for Medicare enrollment was either disability or ESRD were excluded from the present study.

The large multistate MCO offers Medicare Advantage Prescription Drug Plan (MAPD) a Part C plan, which includes the benefits of Medicare Part A, Part B and Part D, to individuals. The MCO insured more than 2.4 million individuals who had continuous MAPD coverage from January 1, 2008 to December 31, 2015. Of these $\geq 2.4$ million individual more than 1.7 million MAPD individuals had "age $\geq 65$ " as the original reason for Medicare enrollment. The remainder 
qualified under Medicare disability or ESRD eligibility provisions. Of these $>1.7$ million members there were 265,554 members who had continuous MAPD coverage with the MCO from January 2008 to December 2015.

The initial sample after the above exclusions of 265,554 members, additional exclusions were: a) individuals who had a prior bariatric surgery (including gastric bypass, laparoscopic gastroenterostomy) $(n=619) ;$ b) individuals with polycystic ovary syndrome $(n=131)$, and lipodystrophy $(n=$ 718); c) individuals with any diagnostic codes related to nephropathy were excluded because it indicates irreversible end organ damage. In the current study, the nephropathy exclusion resulted in excluding $41.3 \%$ of the study sample. Previous research suggested that the rate of chronic kidney disease (CKD) in individuals who are 65 years and older was $61 \%$. CKD rate in the current study was likely lower because individuals with "disability" and "ESRD" as their initial reason for Medicare enrollment were excluded from the sample (155); d) individuals who had pharmacy claims related to drug and/or steroid induced diabetes were excluded $(n=3,436)$; e) individuals with type 1 diabetes were identified using ICD codes and excluded because type 1 diabetes has a different etiology from T2DM $(n=25,535)$; $f$ ) individuals who had T2DM related diagnostics codes only during inpatient hospital stay and did not have T2DM diagnostic claims or T2DM related pharmacy claims in a non-hospital setting were excluded to account for potential surgery induced dysglycemia $(n=1,772)$; g) certain restricted groups as required by the MCO's privacy and legal 
agreements were also excluded from this study. The MCO's IRB required this exclusion $(n=2,238)$.

The definition of "remission" in this study was different from that used by ADA, because of the lack of $\mathrm{HbA} 1_{\mathrm{c}}$ data. An individual was encoded as being in "remission" if they met the following criteria: a) had two or more T2DM related claims in previous years; b) had no T2DM related claims in the given year; c) upon "remission" T2DM individuals were required to continue to have no T2DM related claims until the end of study period, 2015. This requirement excluded individuals who experienced T2DM relapse. This study's focus was only on individuals who experienced continued "remission" and not intermittent "remission."

Individuals were required to have T2DM in the year 2008, 2009, 2010 and 2011 and either continue to be diabetic (no "remission" group) or achieve "remission" (the "remission" group) to be included in the T2DM "remission" study cohort. T2DM relapse can happen in some individuals after "remission." Individuals who had relapse after first "remission" were excluded from the study. The final population included a total of 10,059. The "remission" group included 500 individuals and 9,559 patients in the no "remission group. Since the entire study cohort had T2DM in 2008, 2009, 2010 and 2011, the baseline year was 2011 (Appendix 5.1). 


\section{Case definitions}

T2DM: T2DM individuals were identified using administrative medical and pharmacy claims with the following criteria: at least two diagnoses of T2DM (International Classification of Diseases, Ninth Revision, Clinical Modification [ICD-9-CM] and/or (International Classification of Diseases, Tenth Revision, Clinical Modification [ICD-10-CM]; at least two pharmacy prescriptions related to T2DM; at least 2 claims (medical or pharmacy) related to T2DM. A minimum of two claims was used in the identification of T2DM to exclude patients who were miscoded and patients who were suspected to have T2DM but were never formally diagnosed.

DCSI: The Diabetes Complications Severity Index (DCSI) models the severity of diabetes complication at a specific point in the patient's life. DCSI is calculated as previously described $(98,99)$. The final output is a patient's score (or index value) with a range from 0 to 13 . An individual with DCSI score of 0 indicates the patient has the least severe diabetes. This DCSI score can be used to group the members into high, medium and low diabetic severity.

Retinopathy: This study used ICD-9 and ICD-10 diagnostic codes to identify diabetic ophthalmologic disease, background retinopathy, other retinopathy, retinal edema, cystoid macular edema/degeneration (CSME), other retinal disorders, proliferative retinopathy, retinal detachment, blindness, vitreous hemorrhage). An individual with any of the above clinical conditions was encoded as having retinopathy. 
Neuropathy: This study used ICD-9 and ICD-10 diagnostic codes to identify diabetic neuropathy, amyotrophy, cranial nerve palsy, mononeuropathy, Charcot's arthropathy, polyneuropathy, neurogenic bladder, autonomic neuropathy, gastroparesis/diarrhea, orthostatic hypotension. An individual with any of the above clinical conditions was encoded as having neuropathy.

Peripheral Vascular Disease (PVD): This study used ICD-9 and ICD-10 diagnostic codes to identify diabetic PVD, other aneurysm lower extremities, PVD, foot wound and complication, claudication, intermittent, embolism/thrombosis lower extremities, gangrene, gas gangrene, ulcer of lower limbs. An individual with any of these clinical conditions was encoded as having PVD.

\section{Covariates}

T2DM related clinical conditions were defined using administrative claims data as described previously using both ICD-9 and ICD-10 codes, where applicable (98). Demographic information (age, gender and race) was based on enrollment data. T2DM related pharmacy and statins use was determined using administrative pharmacy claims data.

\section{Statistical Analysis}

Enrollment, medical and pharmacy claims data from 2008 to 2015 of more than 2.4 million members were included in the data aggregation. The data aggregation was done in Hadoop (using Spark, Scala and Hive). Hadoop is an open source software framework for distributed processing and distributed 
storage of very large datasets (151). Processing was done in Hadoop because of the volume of the data included. The analytical dataset was created in a counting process input style $(152,153)$ Means and frequencies to generate member profile were calculated in Hadoop. This data was then moved into SAS 9.2. (SAS Institute Inc., Cary NC) to calculate rate for T2DM "remission." Cox proportional hazard models were specified to identify the significant variables associated with T2DM "remission." SAS procedure PROC PHREG was used for Cox proportional hazard models (152). The adjusted model included demographic variables (gender, and race), statin use, clinical conditions (hypertension, hyperlipidemia, retinopathy, neuropathy, other chronic ischemic heart disease, other atherosclerotic cardiovascular disease and peripheral vascular disease) and DCSI score. The level of significance for all statistical tests was $p<0.05$. 


\section{Results}

There were 10,059 MAPD individuals who had T2DM in 2008, 2009, 2010 and 2011. In the study period there were $4.97 \%(95 \% \mathrm{Cl}, 3.05 \%-8.00 \%)$ Medicare patients 65 years and older $(n=500)$ who met the definition of T2DM "remission" in the study cohort. At 2011, the baseline year, the average age of the "remission" group was 73.7 years, while that of the non- "remission" group was 72.6 years. In the "remission" group $62.2 \%$ were female, compared to $57.2 \%$ in the non-"remission" group. $63.6 \%$ of the "remission" group did not use any diabetes-related medications prior to going into "remission (Table 5.1).

At baseline, the T2DM "remission" group had significantly lower disease prevalence of hypertension, hyperlipidemia, retinopathy (overall, diabetic ophthalmologic disease, background retinopathy, retinal edema, and blindness), neuropathy (overall, diabetic neuropathy, polyneuropathy) and diabetic peripheral vascular disease. However, the "remission" group had higher cardiovascular disease (overall, myocardial infarction, aortic aneurysm and dissection) $(p<0.05)$ (Table 5.2).

In bivariate and multivariate analyses, T2DM "remission" was associated with no statin use, low diabetes complications severity index score, no hypertension, no hyperlipidemia, no neuropathy, no retinopathy, race (Asian/Hispanic), other chronic ischemic heart disease (IHD) and female gender $(p<0.05)$. 
In bivariate analysis "remission" was associated with ulcer of lower limbs and peripheral vascular disease (PVD). However, these associations were attenuated in multivariate analyses and were not significant $(p>0.05)$ (Figure 5.1, Figure 5.2 and Appendix 5.2).

\section{Conclusions}

In Medicare patients 65 years and older, T2DM "remission" does occur without bariatric surgery. $4.97 \%(95 \% \mathrm{Cl}, 3.05 \%-8.00 \%)$ of Medicare patients 65 years and older met the definition of T2DM "remission" in the study cohort. This was not significantly different from the $2.2 \%(1.34 \%-3.54 \%)$ of the 2014 Kaiser Permanente report on $\geq 65$ year old population (20). It is significantly different from the Look AHEAD study which reported T2DM remission incidence of $11.5 \%(95 \% \mathrm{Cl}, 10.1 \%-12.8 \%)$ during the first year and $7.3 \%(95 \% \mathrm{Cl}, 6.2 \%$ $8.4 \%)$ at 4 years in the intensive lifestyle intervention group. The percent "remission" in the present study did not differ from the $2.0 \%$ remission reported for the diabetes support and education control condition (DSE) group $(P<0.001)$ (19). The study presented here found that T2DM "remission" indeed occurs in Medicare patients 65 years and older who did not undergo bariatric surgery. These findings are consistent with previous findings and challenge the widespread notion that T2DM is irreversible in non-bariatric settings.

Consistent with the 2014 Kaiser and the Look AHEAD studies, this study also found that the absence of hyperlipidemia was associated with T2DM "remission." As suggested in previous findings, hyperlipidemia, obesity, and impaired glucose tolerance may have common biological pathway commonly 
called metabolic syndrome. These findings provide additional evidence of the role of managing impaired lipid metabolism to slow or reverse T2DM progression.

Further, this study evaluated multiple clinical conditions related to macrovascular and microvascular comorbidities that are typically associated with T2DM. After adjusting for covariates this study found a number of variables associated with T2DM "remission" that were not previously reported: no statin use; low diabetes complications severity index score; no hypertension; no neuropathy; no retinopathy; race (non-white and non-African American); presence of other chronic ischemic heart disease $(\mathrm{IHD})$ and females $(\mathrm{p}<0.05)$.

Gender: Globally, males are more likely to have T2DM than females $(157,158)$. This trend has changed over the last 50 years. Previously, females were more likely to have diagnosed T2DM, but in recent years, the increasing sedentary lifestyle of males is attributed to their increased prevalence of T2DM $(159,160)$. In line with these studies the current study found that females were more likely to have a T2DM "remission" compared to males.

Race: Consistent with previous studies the current study found that Hispanics and other non-white race (excluding African American) were more like to have a T2DM "remission" in the Medicare 65 years and older population $(p<0.05)(161)$. This finding emphasizes the need for public health policies to develop culturally sensitive interventions to help ameliorate racial disparities of T2DM disease management especially in the elderly population. 
Hypertension: Previous studies indicated that hypertension and T2DM generally occur together and share several common risk factors such as obesity, inflammation, and insulin resistance. Hypertension and T2DM also share common pathways such as those associated with sympathetic nervous system (SNS) and renin-angiotensin-aldosterone system (RAAS) (164). The present study found that even in Medicare patients 65 years and older population the absence of hypertension is associated with T2DM "remission" $(p<0.05)$.

Statins: This study evaluated the role of statins on T2DM "remission" and found that the absence of statin use is associated with T2DM "remission" $(p<0.05)$. There is no previous literature on the role of statin in T2DM "remission." However, its role on T2DM onset is well established. Statin therapy is associated with a 9\% increased risk of T2DM (163). Prior investigation also established that the level of statin potency is associated with T2DM onset (182).

DCSI score: The complications associated with T2DM usually develop simultaneously or consecutively rather than independently. A simple indicator for T2DM related complications may not have been a sufficient measure of T2DM disease progression. Therefore, in the present study, we used an indicator (or score), as previously described, to quantify the severity of diabetic complications $(97,98)$. This indicator is called the Diabetes Complications Severity Index (DCSI) and models the severity of diabetes complication at any given point of time in the patient's life. Using the DCSI score, we further analyzed the association between severity of T2DM and T2DM "remission," and found that lower DCSI scores (i.e., individuals who have less severe diabetes) are 
associated with T2DM "remission." This finding is important because DCSI can thus help in stratifying individuals with T2DM into groups based on score severity. This information can later be used for selecting individuals into disease management programs.

Retinopathy: Retinopathy is one of the most common microvascular damage sites associated with T2DM. Diabetic retinopathy is a major public health problem, with approximately one third of T2DM patients developing some form of retinopathy (183). The healthy function of retina depends on availability of high amounts of oxygen, which in turn depends on intact blood-retinal barrier. The dysglycaemia associated with T2DM causes damage to this barrier by damaging both the inner retinal barrier and/or causing microvascular occlusions. The severity of such retinopathy might range from background retinopathy to complete blindness depending on the level of damage (184). Previous studies have recommended individuals with T2DM to control their blood sugar, blood pressure, and body fat to control the risk of development of advanced retinopathy (183).

Evidence in the present study found that the absence of any T2DM retinopathy was associated with T2DM "remission $(p<0.05)$. Taken in combination with previous recommendations, this study suggests that providers must educate T2DM about the possibility of "remission" by lifestyle modifications a self-incentive for enabling modified behavior, and that they still have a chance to reverse the progression of T2DM. 
Neuropathy: Neuropathy and diabetes are generally associated together. More than $50 \%$ of T2DM individuals have neuropathy and more than $50 \%$ of individuals with neuropathy have T2DM (89). The study presented here found that the absence of neuropathy was associated with T2DM "remission" in Medicare patients 65 years and older.

Neuropathy can be associated with several causes, including metabolic injury (hyperglycemia), compressive injury, ischemic injuries, inflammatory and/or immune response, and other comprehensive etiologies (185). This study did not evaluate the specific reason associated with neuropathy due to the lack of access to physician notes. In spite of this limitation, this finding provides valuable insight into designing public health interventions; for example, individuals with T2DM and without neuropathy may be educated about this finding to incentivize them to make behavior and lifestyle changes.

Other Chronic IHD: IHD is the loss of elasticity and thickening of the coronary arteries, leading to progressive arterial insufficiency. Individuals with "other chronic IHD" were more likely to go into T2DM "remission." This association is not completely understood. One explanation of this association is that there may be a small proportion of individuals in the study sample where the provider takes them off their diabetic medication due to a variety of reasons, such as, the caregiver is less aggressive in motivating patients to achieve lower $\mathrm{HbA} 1_{c}$ levels. Another explanation for this association is that individuals who have certain clinical conditions, such as those related to cardiovascular diseases, might be on constant alert to make healthier choices, especially due to the constant 
reminders that they might be receiving from their provider, insurance agency. These factors, and others, may be the reason why these individuals have a different outcome compared to those without this clinical condition.

There were several limitations to this study. First and most important, the study does not include clinical and laboratory findings such as physician notes and $\mathrm{HbA} 1_{\mathrm{c}}$ lab results, which would have provided a more accurate measure of T2DM "remission." Therefore, a small proportion of individuals could have been asked by their doctors to stop taking T2DM medication for a variety of unknown reasons as noted. The probability of this occurring in the data is very low because even in those cases the physician will probably capture the T2DM related diagnostic code on the medical claims. Also, as with other similar retrospective studies there is a potential for error in information coded in the administrative data. As in other retrospective studies it is not possible to establish causation with such an analytic approach. The level or potency of the T2DM medications including statins was not evaluated in this analysis. DCSI is an unweighted composite index, and does not independently test individual outcomes associated with each complication associated with T2DM. Metabolic syndrome is also excluded from the study due to lack of data such as BMl and physician notes.

In conclusion, T2DM "remission" in Medicare patients 65 years and older is observed in a community setting in a small proportion of individuals who did not undergo bariatric surgery. In majority of the remaining patients T2DM may still remain a major burden and impact quality of life. This study helps establish 
additional evidence to the role of lifestyle intervention in T2DM progression and provides some insights into the possibility of using behavior and lifestyle interventions as alternate pathways for diabetes treatment and management.

Lifestyle interventions such as increased physical activity, low carbohydrate diet, cessation of tobacco smoking, and reducing alcohol intake are already recommended by providers to T2DM patients as a means to control glucose, blood pressure and cholesterol. These study results can further help providers discuss disease prognosis with their T2DM patients and in designing efficacious interventions to the right individuals at the right time. This will help design better T2DM management programs. For example, if a Medicare patient 65 years and older individual is diagnosed with T2DM and has not yet developed any chronic comorbidities such as neuropathy or retinopathy, but has modifiable comorbidities such as hypertension and hyperlipidemia, then their provider can use the insights provided in this study to advise their patients about their possibility to control their T2DM progression by participating in lifestyle intervention programs. DCSI score can be used as an index to further tailor these management programs.

These findings also have significant public health implications ranging from health policy, clinical guidance design, built environment and incentives to enable the success of these lifestyle intervention programs. Lastly, this study recommends further investigation into the lifestyle pathway for T2DM "remission" to improve our understanding of this beneficial alternative. Thus, it may be 
possible to increase the percent of individuals who might benefit from T2DM "remission."

Note: The tables and figures are placed at the end of the article as per the requirement of the journal. 


\section{Tables and Figure Legends}

Table 5.1: Baseline demographic and T2DM "remission" characteristics

\begin{tabular}{|c|c|c|c|}
\hline Characteristic & $\begin{array}{c}\text { No "Remission"* } \\
(n=9,559)\end{array}$ & $\begin{array}{c}\text { "Remission" } \dagger \\
(n=500)\end{array}$ & $P$ value \\
\hline Age, mean (SD), years & $72.6(5.1)$ & $73.7(5.5)$ & $<.0001$ \\
\hline Female sex & $57.2 \%$ & $62.2 \%$ & 0.0273 \\
\hline Race/ethnicity & & & 0.3542 \\
\hline White & $7,679(80.4)$ & 397 (79.6) & \\
\hline Black & $1,378(14.4)$ & $68(13.6)$ & \\
\hline Others & $497(5.2)$ & $34(6.8)$ & \\
\hline
\end{tabular}

Data are represented as $n(\%)$, unless otherwise mentioned

*Missing data in no "remission" cohort: Race $(n=5)$

†Missing data in "remission" cohort: Race $(n=1)$ 
Table 5.2: Baseline comorbidities

\begin{tabular}{|c|c|c|c|}
\hline Characteristic & $\begin{array}{c}\text { No } \\
\text { "Remission"* } \\
(\mathrm{n}=9,559)\end{array}$ & $\begin{array}{c}\text { "Remission" } \dagger \\
(\mathrm{n}=500)\end{array}$ & P value \\
\hline Hypertension & $8,285(86.7)$ & $411(82.2)$ & 0.0040 \\
\hline Hyperlipidemia & $8,125(85.0)$ & $402(80.4)$ & 0.0050 \\
\hline Retinopathy & $1,187(12.4)$ & $32(6.4)$ & $<0.0001$ \\
\hline Diabetic ophthalmologic disease & $830(8.7)$ & $17(3.4)$ & $<0.0001$ \\
\hline Background retinopathy & $392(4.1)$ & $<10$ & 0.0010 \\
\hline Other retinopathy & $138(1.4)$ & $<10$ & 0.6550 \\
\hline Retinal edema & $67(0.7)$ & $<10$ & 0.0600 \\
\hline \multicolumn{4}{|l|}{ Cystoid macular } \\
\hline edema/degeneration & $64(0.7)$ & $<10$ & 0.2010 \\
\hline Other retinal disorders & $40(0.4)$ & $<10$ & 0.9500 \\
\hline Proliferative retinopathy & $61(0.6)$ & $<10$ & 0.0730 \\
\hline Retinal detachment & $72(0.8)$ & $<10$ & 0.1550 \\
\hline Blindness & $55(0.6)$ & $<10$ & 0.0220 \\
\hline Vitreous hemorrhage & $21(0.2)$ & $<10$ & 0.4110 \\
\hline Neuropathy & $1,801(18.8)$ & $68(13.6)$ & 0.0030 \\
\hline Diabetic neuropathy & $1,395(14.6)$ & $46(9.2)$ & 0.0010 \\
\hline Mononeuropathy & $356(3.7)$ & 19 (3.8) & 0.9310 \\
\hline Charcot's arthropathy & $<10$ & $<10$ & 0.1870 \\
\hline Polyneuropathy & $820(8.6)$ & $20(4.0)$ & $<0.0001$ \\
\hline Neurogenic bladder & $15(0.2)$ & $<10$ & 0.8140 \\
\hline Autonomic neuropathy & $78(0.8)$ & $<10$ & 0.3070 \\
\hline Gastroparesis/diarrhea & $44(0.5)$ & $<10$ & 0.3950 \\
\hline Orthostatic hypotension & $145(1.5)$ & $10(2.0)$ & 0.3930 \\
\hline Cerebrovascular & $841(8.8)$ & $42(8.4)$ & 0.7590 \\
\hline Transient ischemic attack (TIA) & $168(1.8)$ & $11(2.2)$ & 0.4660 \\
\hline Stroke & 759 (7.9) & $38(7.6)$ & 0.7840 \\
\hline Cardiovascular & $2,925(30.6)$ & $174(34.8)$ & 0.0470 \\
\hline Atherosclerosis & $572(6.0)$ & $36(7.2)$ & 0.2660 \\
\hline Other ischemic heart disease & $112(1.2)$ & $<10$ & 0.3900 \\
\hline Angina pectoris & 309 (3.2) & $16(3.2)$ & 0.9680 \\
\hline \multicolumn{4}{|l|}{ Other chronic ischemic heart } \\
\hline disease & $1,846(19.3)$ & $94(18.8)$ & 0.7770 \\
\hline Myocardial infarction & $81(0.8)$ & $10(2.0)$ & 0.0080 \\
\hline Ventricular fibrillation, arrest & $732(7.7)$ & $43(8.6)$ & 0.4410 \\
\hline Atrial fibrillation, arrest & $11(0.1)$ & $<10$ & 0.5920 \\
\hline \multicolumn{4}{|l|}{ Other atherosclerotic } \\
\hline cardiovascular disease & $138(1.4)$ & $10(2.0)$ & 0.3140 \\
\hline Old myocardial infarction & $462(4.8)$ & $25(5.0)$ & 0.8650 \\
\hline Heart failure & $503(5.3)$ & $34(6.8)$ & 0.1360 \\
\hline Atherosclerosis, severe & $<10$ & $<10$ & 0.6090 \\
\hline Aortic aneurysm/dissection & $121(1.3)$ & $17(3.4)$ & $<0.0001$ \\
\hline Peripheral vascular disease & $1,074(11.2)$ & $60(12.0)$ & 0.5980 \\
\hline
\end{tabular}




\begin{tabular}{|c|c|c|c|}
\hline Characteristic & $\begin{array}{c}\text { No } \\
\text { “Remission"* } \\
(n=9,559)\end{array}$ & $\begin{array}{c}\text { "Remission" } \\
(n=500)\end{array}$ & $P$ value \\
\hline Diabetic PVD & $524(5.5)$ & $16(3.2)$ & 0.0270 \\
\hline Other aneurysm, lower extremity & $<10$ & $<10$ & 0.0090 \\
\hline Peripheral vascular disease & $807(8.4)$ & $51(10.2)$ & 0.1700 \\
\hline Foot wound + complication & $<10$ & $<10$ & 0.7460 \\
\hline $\begin{array}{l}\text { Claudication, intermittent } \\
\text { Embolism/thrombosis (lower }\end{array}$ & $623(6.5)$ & $46(9.2)$ & 0.0190 \\
\hline extremity) & $<10$ & $<10$ & 0.0440 \\
\hline Gangrene & $<10$ & $<10$ & 0.6470 \\
\hline Gas gangrene & $<10$ & $<10$ & 0.8190 \\
\hline Ulcer of lower limbs & $98(1.0)$ & $<10$ & 0.3530 \\
\hline Metabolic & $46(0.5)$ & $<10$ & 0.3230 \\
\hline Ketoacidosis & $16(0.2)$ & $<10$ & 0.8630 \\
\hline Hyperosmolar & $22(0.2)$ & $<10$ & 0.4480 \\
\hline Other coma & $<10$ & $<10$ & 0.3960 \\
\hline
\end{tabular}

Data are expressed as $n$ (\%). Baseline year $=2011$

If the number of individuals in a cell were less than 10 , the number is replaced

with "<10" 
Figure 5.1: Unadjusted Cox proportional hazard for T2DM "remission"

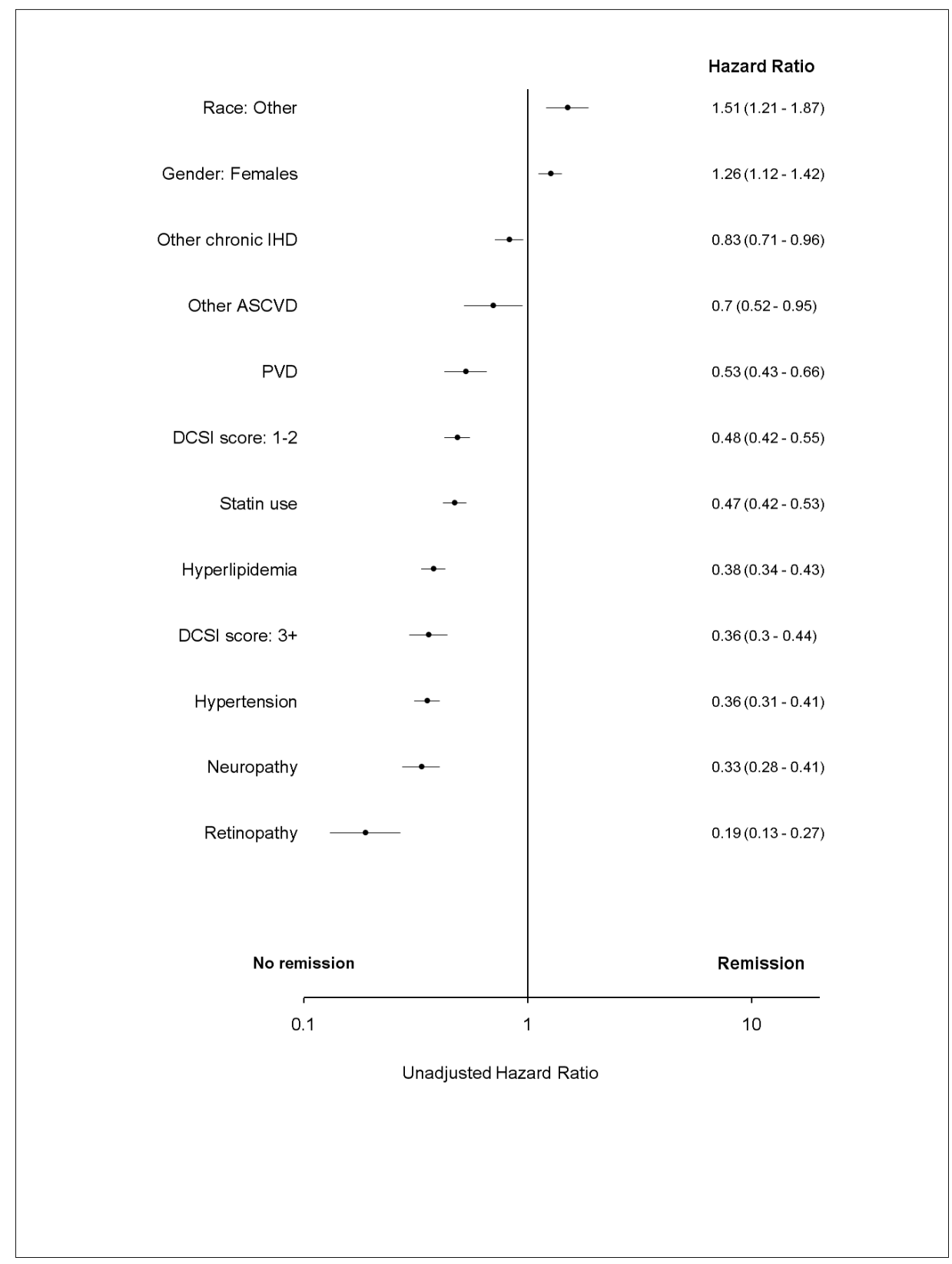

ASCVD, atherosclerotic cardiovascular disease; DCSI, Diabetes complications severity index; IHD, ischemic heart disease. Race: other, non-white and non-African American; PVD, peripheral vascular disease. 
Figure 5.2: Adjusted Cox proportional hazard for T2DM "remission"

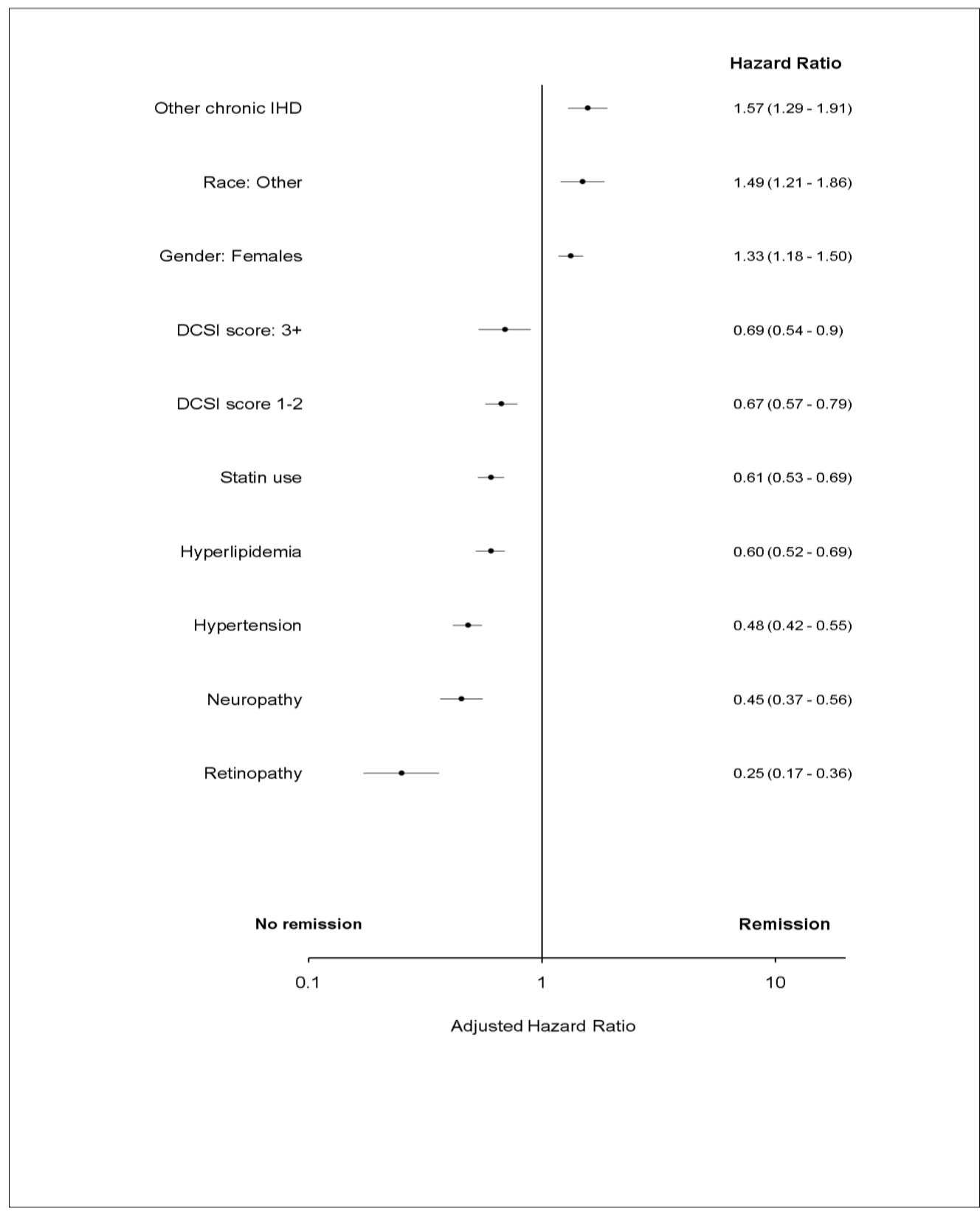

DCSI: Diabetes complications severity index; IHD, ischemic heart disease;

Race: other, non-white and non-African American. 
Appendix: Table 5.1: T2DM "remission" definitions

T2DM Diagnosis

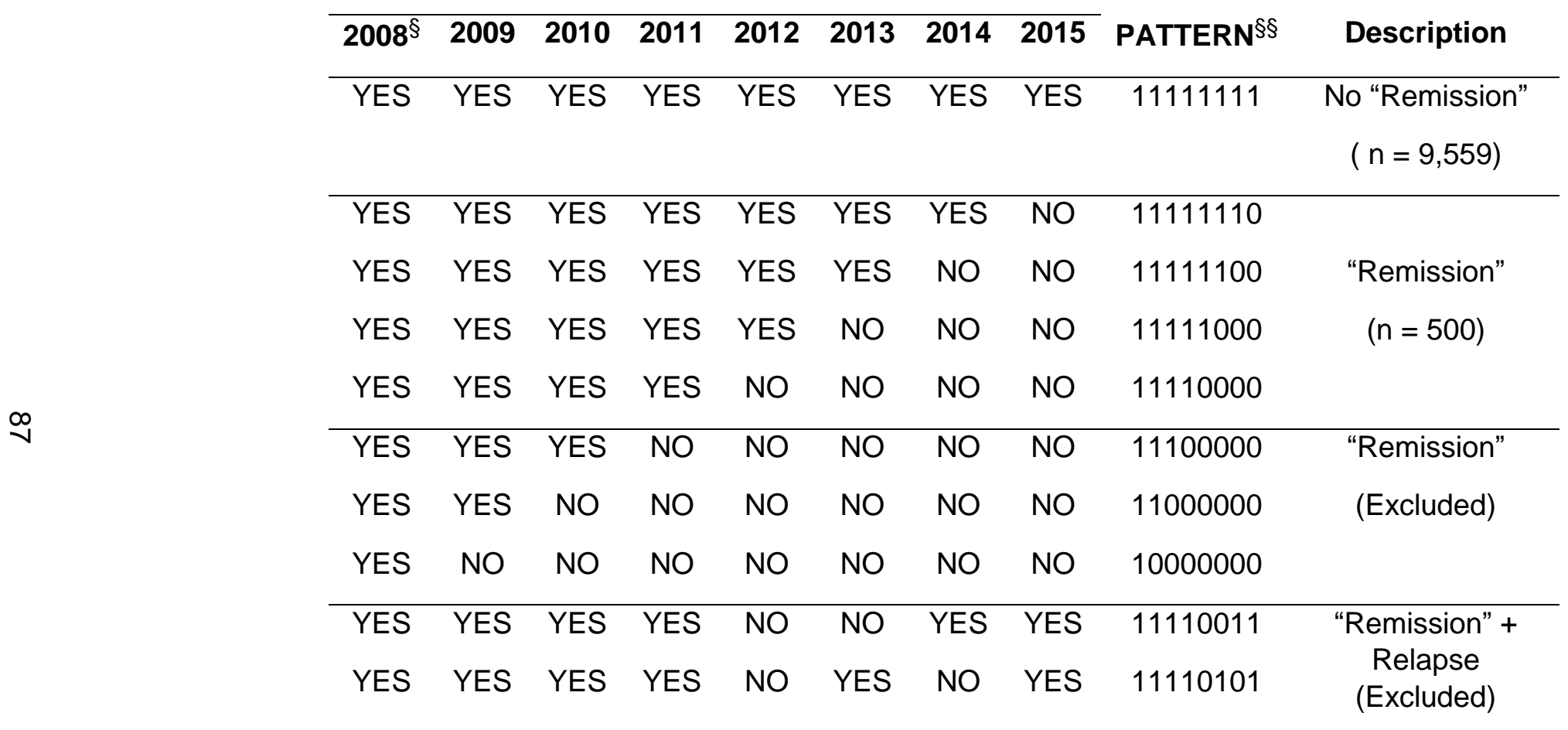

$\S N O=$ no type 2 diabetes diagnosis, and YES = type 2 diabetes diagnosis

$\S \S_{0}=$ no $\mathrm{T} 2 \mathrm{DM}$ and $1=$ presence of $\mathrm{T} 2 \mathrm{DM}$ 
Appendix: Table 5.2 - Unadjusted and adjusted Cox proportional hazard for "remission"

\begin{tabular}{|c|c|c|c|c|c|}
\hline \multirow[b]{3}{*}{ Characteristic } & \multicolumn{4}{|c|}{ Any "Remission" $(n=500)$} & \multirow[b]{3}{*}{$P$ value } \\
\hline & \multicolumn{4}{|c|}{ Hazard Ratio $(95 \% \mathrm{Cl})$} & \\
\hline & n (\%) & Unadjusted & $\mathrm{P}$ Value & Fully adjusted & \\
\hline \multicolumn{6}{|l|}{ Gender } \\
\hline Males & $189(4.4)$ & 1.0 (ref) & & $1.0(\mathrm{ref})$ & $<0.0001$ \\
\hline Females & $311(5.3)$ & $1.26(1.12-1.42)$ & $<0.0001$ & $1.33(1.18-1.50)$ & \\
\hline \multicolumn{6}{|c|}{ 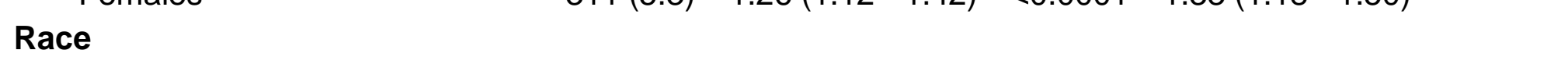 } \\
\hline White & $397(4.92)$ & 1.0 (ref) & & 1.0 (ref) & \\
\hline Black & $68(4.7)$ & $0.93(0.78-1.10)$ & 0.3936 & $1.01(0.85-1.20$ & 0.8849 \\
\hline Other & $34(6.4)$ & $1.51(1.21-1.87)$ & 0.0002 & $1.49(1.21-1.86)$ & 0.0002 \\
\hline \multicolumn{6}{|l|}{ DCSI Score } \\
\hline Zero & $293(7.7)$ & $1.0(\mathrm{ref})$ & $<0.0001$ & $1.0(\mathrm{ref})$ & $<0.0001$ \\
\hline $1-2$ & $152(3.7)$ & $0.48(0.42-0.55)$ & & $0.67(0.57-0.79)$ & \\
\hline $3+$ & $55(2.6)$ & $0.36(0.29-0.44)$ & & $0.69(0.54-0.89)$ & \\
\hline \multicolumn{6}{|l|}{ Statins } \\
\hline No statins & $145(9.32)$ & 1.0 (ref) & & $1.0(\mathrm{ref})$ & $<0.0001$ \\
\hline Statins & $355(4.17)$ & $0.47(0.42-0.53)$ & $<0.0001$ & $0.61(0.53-0.69)$ & \\
\hline \multicolumn{6}{|l|}{ Clinical Conditions } \\
\hline No hypertension & $33(13.92)$ & 1.0 (ref) & & 1.0 (ref) & $<0.0001$ \\
\hline Hypertension & $467(4.75)$ & $0.36(0.31-0.41)$ & $<0.0001$ & $0.48(0.42-0.55)$ & \\
\hline No hyperlipidemia & $25(9.43)$ & $1.0(\mathrm{ref})$ & & $1.0(\mathrm{ref})$ & $<0.0001$ \\
\hline Hyperlipidemia & $475(4.85)$ & $0.38(0.34-0.43)$ & $<0.0001$ & $0.60(0.52-0.69)$ & \\
\hline \multicolumn{6}{|c|}{ 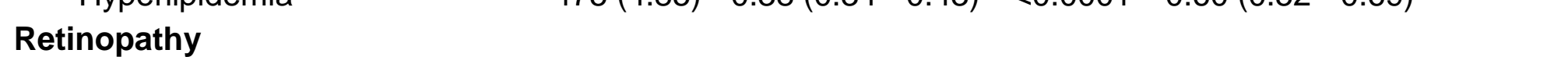 } \\
\hline No retinopathy & $411(6.39)$ & 1.0 (ref) & $<0.0001$ & 1.0 (ref) & $<0.0001$ \\
\hline Retinopathy & $89(2.45)$ & $0.19(0.13-0.27)$ & & $0.25(0.17-0.36)$ & \\
\hline \multicolumn{6}{|l|}{ Neuropathy } \\
\hline No neuropathy & $322(6.85)$ & 1.0 (ref) & $<0.0001$ & $1.0(\mathrm{ref})$ & $<0.0001$ \\
\hline
\end{tabular}




\begin{tabular}{|c|c|c|c|c|c|}
\hline \multirow[b]{3}{*}{ Characteristic } & \multicolumn{4}{|c|}{ Any "Remission" $(n=500)$} & \multirow[b]{3}{*}{$P$ value } \\
\hline & \multicolumn{4}{|c|}{ Hazard Ratio (95\% Cl) } & \\
\hline & n (\%) & Unadjusted & $P$ Value & Fully adjusted & \\
\hline Neuropathy & $178(3.32)$ & $0.33(0.28-0.41)$ & & $0.45(0.37-0.56)$ & \\
\hline \multicolumn{6}{|l|}{ Cardiovascular } \\
\hline No other ASCVD & $476(5.49)$ & 1.0 (ref) & 0.0202 & 1.0 (ref) & 0.3796 \\
\hline Other ASCVD & $24(1.74)$ & $0.70(0.52-0.95)$ & & $0.86(0.62-1.19)$ & \\
\hline No other chronic IHD & $340(5.45)$ & 1.0 (ref) & 0.0106 & 1.0 (ref) & $<0.0001$ \\
\hline Other Chronic IHD & $160(4.19)$ & $0.83(0.71-0.96)$ & & $1.57(1.29-1.91)$ & \\
\hline \multicolumn{6}{|l|}{ Peripheral vascular Disease } \\
\hline No peripheral vascular disease & $380(5.65)$ & 1.0 (ref) & $<0.0001$ & 1.0 (ref) & 0.1625 \\
\hline Peripheral vascular disease & $120(3.6)$ & $0.53(0.43-0.66)$ & & $0.85(0.67-1.07)$ & \\
\hline
\end{tabular}

Fully adjusted model includes all variables in this table

*Missing data in No "remission" cohort: race $(n=5)$, in "remission" cohort: race $(n=1)$

If the number of Individuals in a cell was $<10$, the number is replaced with " $<10$ "

Data represented as $\mathrm{n}(\%)$ for baseline year (2011) 


\section{CONCLUSIONS}

Type 2 diabetes mellitus is a progressive chronic disease condition. If left unmanaged, the body's ability to regulate blood sugar deteriorates gradually. The complications associated with dysglycemia can have deleterious effects on the quality of life of the patient. In order to develop effective intervention programs, it is first important to understand the prognosis of T2DM. In this regards, the study presented here investigated two key sub components of T2DM progression: a) onset of T2DM; b) "remission" of T2DM in a non-bariatric setting.

Using two different study cohorts the study presented here identified key factors that are associated with T2DM onset and "remission" in Medicare patients 65 years and older. The factors that were significantly associated with T2DM onset were: male gender; non-white ethnicity (African American, Hispanics); statin use; hypertension; hyperlipidemia; heart failure; ulcer of lower limbs; atherosclerosis; other retinopathy; angina pectoris; blindness and low vision; absence of other chronic ischemic heart disease (IHD) $(p<0.05)$. The factors associated with T2DM "remission" were no statin use; low diabetes complications severity index score (DCSI); no hypertension; no hyperlipidemia; no neuropathy; no retinopathy $(p<0.05)$. Race $($ Asian/Hispanic); other chronic ischemic heart

disease (IHD); and female gender were significantly associated with T2DM "remission" $(p<0.05)$. 
The combined results of the onset and "remission" study suggests that hypertension, hyperlipidemia, statin use, gender, and race are some of the common factors associated with both T2DM onset and "remission." Hypertension and hyperlipidemia are key components of metabolic syndrome. Statins are used for cardiovascular disease risk reduction in patients with metabolic syndrome (186). These findings suggest that further investigation in the role of metabolic syndrome in T2DM progression is warranted for future studies.

This study also found that lower DCSI scores were associated with T2DM "remission." This finding can help in stratifying individuals with T2DM into groups based on their diabetes severity. This information can thus be used for selecting individuals into disease management programs.

The sociodemographic and clinical profile of Medicare patients 65 years and older with T2DM onset and T2DM "remission" could be used to: a) educate T2DM patients, providers, payors and other stakeholders; b) develop T2DM management programs; c) design public health policies. This will in turn help to improve quality of life and reduce T2DM disease burden. 


\section{Limitations}

There were several limitations to this study as described below.

a) Lack of data: First and most important, the study does not include clinical and laboratory findings such as physician notes and $\mathrm{HbA} 1_{\mathrm{c}}$, which would have provided a more accurate measure of T2DM onset and T2DM remission. This is especially important in the "remission" cohort because there could be a small proportion of individuals who were asked by their doctors to stop taking T2DM medication in order to decrease the stress on the body, or the individual might have other reasons to stop taking medication, or get prescription samples for diabetes medication, or pay out of pocket for their T2DM medication. The probability of not identifying individual's T2DM status due to these reasons would be low because even in the absence of pharmacy claims, an individual's medical claims are often captured as diagnostic on the medical claims.

Data such as nutrition, physical activity, BMI, original diagnosis date for T2DM are also not available. The study does not further split the study cohort based on pre-diabetes and/or undiagnosed individuals.

Individuals with metabolic syndrome cannot be identified due to lack of data such as BMI and cholesterol data.

b) Coding error: As with other similar retrospective studies there is a potential for error in the information coded on the administrative data. Even though the claims are audited on a regular basis there is a possibility of potential for error in the information coded on medical claims due to provider error, fraud etc. 
c) Causation: As with similar studies, this type of analysis can only establish associations and is not intended to establish causation of T2DM onset or "remission".

d) Complexity: This study is a quasi-experiment (i.e., it's not a true randomized study). The factors (covariates, confounding variables, etc.) will not be random and will not be able to completely eliminate bias or confounding.

e) Dosage of T2DM drug and statin: The level or potency of the T2DM medications and statins was not evaluated in this analysis.

f) DCSI score: is an unweighted composite index, and does not independently test individual outcomes associated with each complication associated with T2DM.

g) Generalizability: The exclusions such as excluding disabled individuals, excluding individuals with ESRD, excluding individuals with chronic kidney function in the study cohort limits the generalizability of the study to the overall Medicare population.

\section{Strengths}

The medical and pharmacy data being studied here are part of a standardized data collection practice of the health insurance organization. The data is collected using a standardized process with standard protocols for extraction, transformation and loading (ETL). The data is audited by the organization for its business purposes and hence less prone to data integrity issues. 


\section{REFERENCES}

1. Kahn SE, Cooper ME, Del Prato S. Pathophysiology and treatment of type 2 diabetes: perspectives on the past, present, and future. Lancet. 2014;

383:1068-83.

2. Cade WT. Diabetes-related microvascular and macrovascular diseases in the physical therapy setting. Phys Ther. 2008; 88:1322-35.

3. Brunetti L, Kalabalik J. Management of type-2 diabetes mellitus in adults: focus on individualizing non-insulin therapies. P T. 2012; 37:687-96.

4. CDC. National Diabetes Statistics Report: Estimates of Diabetes and Its Burden in the United States. In: Services USDoHaH, editor. Atlanta, GA2014. 5. American Diabetes A. Economic costs of diabetes in the U.S. in 2012. Diabetes Care. 2013; 36:1033-46.

6. Fletcher B, Gulanick M, Lamendola C. Risk factors for type 2 diabetes mellitus. J Cardiovasc Nurs. 2002; 16:17-23.

7. CDC. Basics About Diabetes. 2016; Available from: http://www.cdc.gov/diabetes/basics/diabetes.html.

8. Choi BC, Shi F. Risk factors for diabetes mellitus by age and sex: results of the National Population Health Survey. Diabetologia. 2001; 44:1221-31. 
9. Bi Y, Wang $\mathrm{T}, \mathrm{Xu} \mathrm{M}, \mathrm{Xu} \mathrm{Y}, \mathrm{Li} \mathrm{M}, \mathrm{Lu} \mathrm{J}$, et al. Advanced research on risk factors of type 2 diabetes. Diabetes/metabolism research and reviews. 2012; 28 Suppl 2:32-9.

10. Wu Y, Ding Y, Tanaka Y, Zhang W. Risk factors contributing to type 2 diabetes and recent advances in the treatment and prevention. Int $\mathrm{J}$ Med Sci. $2014 ; 11: 1185-200$.

11. Zivanovic D, Sipetic S, Stamenkovic-Radak M, Milasin J. [Potential risk factors for developing diabetes mellitus type 2]. Med Pregl. 2010; 63:231-6.

12. Emdin CA, Anderson SG, Woodward M, Rahimi K. Usual Blood Pressure and Risk of New-Onset Diabetes: Evidence From 4.1 Million Adults and a MetaAnalysis of Prospective Studies. J Am Coll Cardiol. 2015; 66:1552-62.

13. Wei PL, Tsai MC, Hung SH, Lee HC, Lin HC, Lee CZ. Risk of new-onset type II diabetes after appendicectomy. Br J Surg. 2015; 102:1267-71.

14. Gronich N, Deftereos SN, Lavi I, Persidis AS, Abernethy DR, Rennert G. Hypothyroidism is a Risk Factor for New-Onset Diabetes: A Cohort Study. Diabetes Care. 2015; 38:1657-64.

15. Colagiuri S, Davies $\mathrm{D}$. The value of early detection of type 2 diabetes. Curr Opin Endocrinol Diabetes Obes. 2009; 16:95-9.

16. Harris MI, Klein R, Welborn TA, Knuiman MW. Onset of NIDDM occurs at least 4-7 yr before clinical diagnosis. Diabetes Care. 1992; 15:815-9.

17. Jarrett RJ. Duration of non-insulin-dependent diabetes and development of retinopathy: analysis of possible risk factors. Diabet Med. 1986; 3:261-3. 
18. Implications of the United Kingdom Prospective Diabetes Study. Diabetes Care. 2002; 25:s28-s32.

19. Gregg EW, Chen H, Wagenknecht LE, Clark JM, Delahanty LM, Bantle J, et al. Association of an intensive lifestyle intervention with remission of type 2 diabetes. JAMA : the journal of the American Medical Association. 2012; 308:2489-96.

20. Karter AJ, Nundy S, Parker MM, Moffet HH, Huang ES. Incidence of remission in adults with type 2 diabetes: the diabetes \& aging study. Diabetes Care. 2014; 37:3188-95.

21. Shrivastava SR, Shrivastava PS, Ramasamy J. Role of self-care in management of diabetes mellitus. J Diabetes Metab Disord. 2013; 12:14.

22. Phillips P. Type 2 Diabetes - failure, blame and guilt in the adoption of insulin therapy. Rev Diabet Stud. 2005; 2:35-9.

23. U.K. prospective diabetes study 16 . Overview of 6 years' therapy of type II diabetes: a progressive disease. U.K. Prospective Diabetes Study Group. Diabetes. 1995; 44:1249-58.

24. Fowler MJ. Microvascular and macrovascular complications of diabetes. Clinical diabetes. 2008; 26:77-82.

25. WHO. Diabetes Programme. 2015.

26. CMS. National Health Expenditures 2014 Highlights 2014.

27. Keehan SP, Cuckler GA, Sisko AM, Madison AJ, Smith SD, Stone DA, et al. National health expenditure projections, 2014-24: spending growth faster than recent trends. Health affairs. 2015; 34:1407-17. 
28. Milstein A, Shortell S. Innovations in care delivery to slow growth of US health spending. JAMA : the journal of the American Medical Association. 2012; 308:1439-40.

29. Milstein A. Code red and blue--safely limiting health care's GDP footprint. The New England journal of medicine. 2013; 368:1-3.

30. OECD. Health Care Systems: OECD Publishing.

31. RWJF. America Is Not Getting Good Value for Its Health Dollar. 2013 [March 2013]; Available from: http://www.rwjf.org/en/topics/rwjf-topic-areas/costand-value.html.

32. Hussey PS, Wertheimer S, Mehrotra A. The association between health care quality and cost: a systematic review. Annals of internal medicine. 2013; 158:27-34.

33. WHO. The determinants of health. 2015; Available from: http://www.who.int/hia/evidence/doh/en/.

34. Schroeder SA. We Can Do Better - Improving the Health of the American People. New England Journal of Medicine. 2007; 357:1221-8.

35. Heiman HJ, Artiga S. Beyond Health Care: The Role of Social Determinants in Promoting Health and Health Equity. Health. 20:10. 36. Braveman P, Gottlieb L. The social determinants of health: it's time to consider the causes of the causes. Public Health Rep. 2014; 129 Suppl 2:19-31. 37. Alamian A, Paradis G. Individual and social determinants of multiple chronic disease behavioral risk factors among youth. BMC Public Health. 2012; $12: 224$. 
38. CDC. The Power of Prevention: Chronic disease ... the public health challenge of the 21st century. 2009.

39. Healthcare.gov. Prevention and Public Health Fund: Community Transformation Grants to Reduce Chronic Disease. 2011; Available from: http://www.healthcare.gov/news/factsheets/2011/05/grants05132011a.html.

40. Steyn K, Damasceno A. Lifestyle and Related Risk Factors for Chronic Diseases. In: Jamison DT, Feachem RG, Makgoba MW, Bos ER, Baingana FK, Hofman KJ, et al., editors. Disease and Mortality in Sub-Saharan Africa. 2nd ed. Washington (DC)2006.

41. Willett WC, Koplan JP, Nugent R, Dusenbury C, Puska P, Gaziano TA. Prevention of Chronic Disease by Means of Diet and Lifestyle Changes. In: Jamison DT, Breman JG, Measham AR, Alleyne G, Claeson M, Evans DB, et al., editors. Disease Control Priorities in Developing Countries. 2nd ed. Washington (DC)2006.

42. Chang HY, Weiner JP, Richards TM, Bleich SN, Segal JB. Predicting costs with diabetes complications severity index in claims data. The American journal of managed care. 2012; 18:213-9.

43. Jordan $\mathrm{CO}$, Slater M, Kottke TE. Preventing chronic disease risk factors: rationale and feasibility. Medicina (Kaunas). 2008; 44:745-50.

44. Mokdad AH, Marks JS, Stroup DF, Gerberding JL. Actual causes of death in the United States, 2000. JAMA : the journal of the American Medical Association. 2004; 291:1238-45. 
45. Bauer UE, Briss PA, Goodman RA, Bowman BA. Prevention of chronic disease in the 21st century: elimination of the leading preventable causes of premature death and disability in the USA. Lancet. 2014; 384:45-52.

46. American Diabetes A. Diagnosis and classification of diabetes mellitus. Diabetes Care. 2010; 33 Suppl 1:S62-9.

47. Maiese K, Morhan SD, Chong ZZ. Oxidative stress biology and cell injury during type 1 and type 2 diabetes mellitus. Curr Neurovasc Res. 2007; 4:63-71. 48. Chronic Disease Prevention and Health Promotion - Diabetes [database on the Internet]2015. Available from:

http://www.cdc.gov/chronicdisease/resources/publications/aag/ddt.htm.

49. Harris MI, Eastman RC. Early detection of undiagnosed diabetes mellitus: a US perspective. Diabetes/metabolism research and reviews. 2000; 16:230-6. 50. Cefalu WT, Petersen MP, Ratner RE. The alarming and rising costs of diabetes and prediabetes: a call for action! Diabetes Care. 2014; 37:3137-8. 51. Olokoba AB, Obateru OA, Olokoba LB. Type 2 diabetes mellitus: a review of current trends. Oman Med J. 2012; 27:269-73.

52. Cohen RM, Haggerty S, Herman WH. HbA1c for the diagnosis of diabetes and prediabetes: is it time for a mid-course correction? J Clin Endocrinol Metab. 2010; 95:5203-6.

53. Fonseca VA. Identification and treatment of prediabetes to prevent progression to type 2 diabetes. Clinical cornerstone. 2007; 8:10-8; discussion 920. 
54. Tuso P. Prediabetes and lifestyle modification: time to prevent a preventable disease. Perm J. 2014; 18:88-93.

55. Twito O, Frankel M, Nabriski D. Impact of glucose level on morbidity and mortality in elderly with diabetes and pre-diabetes. World J Diabetes. 2015; 6:345-51.

56. Wilmot E, Idris I. Early onset type 2 diabetes: risk factors, clinical impact and management. Ther Adv Chronic Dis. 2014; 5:234-44.

57. Brancati FL, Whelton PK, Kuller LH, Klag MJ. Diabetes mellitus, race, and socioeconomic status. A population-based study. Ann Epidemiol. 1996; 6:67-73. 58. Bernstein JA, McCloskey L, Gebel CM, Iverson RE, Lee-Parritz A. Lost opportunities to prevent early onset type 2 diabetes mellitus after a pregnancy complicated by gestational diabetes. BMJ Open Diabetes Res Care. 2016; 4:e000250.

59. Engum A. The role of depression and anxiety in onset of diabetes in a large population-based study. J Psychosom Res. 2007; 62:31-8.

60. Betteridge DJ, Carmena R. The diabetogenic action of statins mechanisms and clinical implications. Nat Rev Endocrinol. 2016; 12:99-110. 61. Banach M, Malodobra-Mazur M, Gluba A, Katsiki N, Rysz J, Dobrzyn A. Statin therapy and new-onset diabetes: molecular mechanisms and clinical relevance. Curr Pharm Des. 2013; 19:4904-12.

62. Aiman U, Najmi A, Khan RA. Statin induced diabetes and its clinical implications. J Pharmacol Pharmacother. 2014; 5:181-5. 
63. Wong J, Molyneaux L, Constantino M, Twigg SM, Yue DK. Timing is everything: age of onset influences long-term retinopathy risk in type 2 diabetes, independent of traditional risk factors. Diabetes Care. 2008; 31:1985-90.

64. Fonseca VA. Defining and characterizing the progression of type 2 diabetes. Diabetes Care. 2009; 32 Suppl 2:S151-6.

65. Rolla AR. Progression of type 2 diabetes and insulin initiation. J Natl Med Assoc. 2011; 103:241-6.

66. Valdivielso P, Ramirez-Bollero J, Perez-Lopez C. Peripheral arterial disease, type 2 diabetes and postprandial lipidaemia: Is there a link? World $\mathrm{J}$ Diabetes. 2014; 5:577-85.

67. Almdal $\mathrm{T}$, Scharling $\mathrm{H}$, Jensen JS, Vestergaard $\mathrm{H}$. The independent effect of type 2 diabetes mellitus on ischemic heart disease, stroke, and death: a population-based study of 13,000 men and women with 20 years of follow-up. Arch Intern Med. 2004; 164:1422-6.

68. Mannucci E, Dicembrini I, Lauria A, Pozzilli P. Is glucose control important for prevention of cardiovascular disease in diabetes? Diabetes Care. 2013; 36 Suppl 2:S259-63.

69. Martin-Timon I, Sevillano-Collantes C, Segura-Galindo A, Del CanizoGomez FJ. Type 2 diabetes and cardiovascular disease: Have all risk factors the same strength? World J Diabetes. 2014; 5:444-70.

70. Carneiro AV. Coronary heart disease in diabetes mellitus: risk factors and epidemiology. Rev Port Cardiol. 2004; 23:1359-66. 
71. Matveyenko A, Vella A. Regenerative Medicine in Diabetes. Mayo Clin Proc. 2015; 90:546-54.

72. Heinonen SE, Genove G, Bengtsson E, Hubschle T, Akesson L, Hiss K, et al. Animal Models of Diabetic Macrovascular Complications: Key Players in the Development of New Therapeutic Approaches. J Diabetes Res. 2015; 2015:404085.

73. Al-Wakeel JS, Hammad D, Al Suwaida A, Mitwalli AH, Memon NA, Sulimani F. Microvascular and macrovascular complications in diabetic nephropathy patients referred to nephrology clinic. Saudi J Kidney Dis Transpl. 2009; 20:77-85.

74. Mooradian AD. Dyslipidemia in type 2 diabetes mellitus. Nat Clin Pract Endocrinol Metab. 2009; 5:150-9.

75. Sowers JR, Khoury S, Standley P, Zemel P, Zemel M. Mechanisms of hypertension in diabetes. Am J Hypertens. 1991; 4:177-82.

76. Yaffe K, Falvey C, Hamilton N, Schwartz AV, Simonsick EM, Satterfield S, et al. Diabetes, glucose control, and 9-year cognitive decline among older adults without dementia. Arch Neurol. 2012; 69:1170-5.

77. Pandey A, Forte V, Abdallah M, Alickaj A, Mahmud S, Asad S, et al. Diabetes mellitus and the risk of cancer. Minerva Endocrinol. 2011; 36:187-209. 78. Ducat L, Philipson LH, Anderson BJ. The mental health comorbidities of diabetes. JAMA : the journal of the American Medical Association. 2014; 312:691-2. 
79. Schernhammer E, Hansen J, Rugbjerg K, Wermuth L, Ritz B. Diabetes and the risk of developing Parkinson's disease in Denmark. Diabetes Care. 2011; 34:1102-8.

80. Cigolle CT, Blaum CS, Halter JB. Diabetes and cardiovascular disease prevention in older adults. Clin Geriatr Med. 2009; 25:607-41, vii-viii.

81. Morgan CL, Currie CJ, Peters JR. Relationship between diabetes and mortality: a population study using record linkage. Diabetes Care. 2000; 23:11037.

82. Burr JF, Shephard RJ, Riddell MC. Prediabetes and type 2 diabetes mellitus: assessing risks for physical activity clearance and prescription. Can Fam Physician. 2012; 58:280-4.

83. Speight J, Sinclair AJ, Browne JL, Woodcock A, Bradley C. Assessing the impact of diabetes on the quality of life of older adults living in a care home: validation of the ADDQoL Senior. Diabet Med. 2013; 30:74-80.

84. Rubin RR, Peyrot M. Quality of life and diabetes. Diabetes/metabolism research and reviews. 1999; 15:205-18.

85. The Effect of Intensive Treatment of Diabetes on the Development and Progression of Long-Term Complications in Insulin-Dependent Diabetes Mellitus. New England Journal of Medicine. 1993; 329:977-86.

86. Fong DS, Aiello L, Gardner TW, King GL, Blankenship G, Cavallerano JD, et al. Retinopathy in diabetes. Diabetes Care. 2004; 27 Suppl 1:S84-7.

87. Diabetic Retinopathy. Diabetes Care. 2002; 25:s90-s3. 
88. Lim A. Diabetic nephropathy - complications and treatment. Int J Nephrol Renovasc Dis. 2014; 7:361-81.

89. Smith AG, Singleton JR. Diabetic neuropathy. Continuum (Minneap Minn). 2012; 18:60-84.

90. Zhou H, Zhang X, Lu J. Progress on diabetic cerebrovascular diseases. Bosn J Basic Med Sci. 2014; 14:185-90.

91. Huysman E, Mathieu C. Diabetes and peripheral vascular disease. Acta Chir Belg. 2009; 109:587-94.

92. Alberti KG, Zimmet P, Shaw J. Metabolic syndrome--a new world-wide definition. A Consensus Statement from the International Diabetes Federation. Diabet Med. 2006; 23:469-80.

93. Zimmet P, Alberti KG, Kaufman F, Tajima N, Silink M, Arslanian S, et al. The metabolic syndrome in children and adolescents - an IDF consensus report. Pediatr Diabetes. 2007; 8:299-306.

94. Wassink AM, Van Der Graaf Y, Soedamah-Muthu SS, Spiering W, Visseren F, Smart Study G. Metabolic syndrome and incidence of type 2 diabetes in patients with manifest vascular disease. Diab Vasc Dis Res. 2008; 5:114-22.

95. Isomaa B, Almgren P, Tuomi T, Forsen B, Lahti K, Nissen M, et al. Cardiovascular morbidity and mortality associated with the metabolic syndrome. Diabetes Care. 2001; 24:683-9. 
96. Abda E, Hamza L, Tessema F, Cheneke W. Metabolic syndrome and associated factors among outpatients of Jimma University Teaching Hospital. Diabetes Metab Syndr Obes. 2016; 9:47-53.

97. Ramsey SD, Newton K, Blough D, McCulloch DK, Sandhu N, Wagner EH. Patient-level estimates of the cost of complications in diabetes in a managedcare population. Pharmacoeconomics. 1999; 16:285-95.

98. Young BA, Lin E, Von Korff M, Simon G, Ciechanowski P, Ludman EJ, et al. Diabetes complications severity index and risk of mortality, hospitalization, and healthcare utilization. The American journal of managed care. 2008; 14:1523.

99. Chang HY, Weiner JP, Richards TM, Bleich SN, Segal JB. Validating the adapted Diabetes Complications Severity Index in claims data. The American journal of managed care. 2012; 18:721-6.

100. Katon WJ, Lin EH, Williams LH, Ciechanowski P, Heckbert SR, Ludman E, et al. Comorbid depression is associated with an increased risk of dementia diagnosis in patients with diabetes: a prospective cohort study. J Gen Intern Med. 2010; 25:423-9.

101. Ciechanowski P, Russo J, Katon WJ, Lin EH, Ludman E, Heckbert S, et al. Relationship styles and mortality in patients with diabetes. Diabetes Care. 2010; 33:539-44.

102. Katon W, Russo J, Lin EH, Heckbert SR, Karter AJ, Williams LH, et al. Diabetes and poor disease control: is comorbid depression associated with poor 
medication adherence or lack of treatment intensification? Psychosom Med. $2009 ; 71: 965-72$.

103. Maciejewski ML, Liu CF, Fihn SD. Performance of comorbidity, risk adjustment, and functional status measures in expenditure prediction for patients with diabetes. Diabetes Care. 2009; 32:75-80.

104. Ludman EJ, Russo JE, Katon WJ, Simon GE, Williams LH, Lin EH, et al. How does change in depressive symptomatology influence weight change in patients with diabetes? Observational results from the Pathways longitudinal cohort. J Gerontol A Biol Sci Med Sci. 2010; 65:93-8.

105. Taylor R. Banting Memorial lecture 2012: reversing the twin cycles of type 2 diabetes. Diabet Med. 2013; 30:267-75.

106. Buse JB, Caprio S, Cefalu WT, Ceriello A, Del Prato S, Inzucchi SE, et al. How do we define cure of diabetes? Diabetes Care. 2009; 32:2133-5. 107. Ades PA, Savage PD, Marney AM, Harvey J, Evans KA. Remission of recently diagnosed type 2 diabetes mellitus with weight loss and exercise. $\mathrm{J}$ Cardiopulm Rehabil Prev. 2015; 35:193-7.

108. ADA. Standards of Medical Care in Diabetes-2016: Summary of Revisions. Diabetes Care. 2016; 39 Suppl 1:S4-5.

109. Zullig LL, Gellad WF, Moaddeb J, Crowley MJ, Shrank W, Granger BB, et al. Improving diabetes medication adherence: successful, scalable interventions. Patient Prefer Adherence. 2015; 9:139-49.

110. Imam K. Management and treatment of diabetes mellitus. Adv Exp Med Biol. 2012; 771:356-80. 
111. Gary TL, Genkinger JM, Guallar E, Peyrot M, Brancati FL. Meta-analysis of randomized educational and behavioral interventions in type 2 diabetes. Diabetes Educ. 2003; 29:488-501.

112. Brown SA. Interventions to promote diabetes self-management: state of the science. Diabetes Educ. 1999; 25:52-61.

113. Renders CM, Valk GD, Griffin SJ, Wagner EH, Eijk Van JT, Assendelft WJ. Interventions to improve the management of diabetes in primary care, outpatient, and community settings: a systematic review. Diabetes Care. 2001; 24:1821-33.

114. Deakin T, McShane CE, Cade JE, Williams RD. Group based training for self-management strategies in people with type 2 diabetes mellitus. Cochrane Database Syst Rev. 2005:CD003417.

115. Norris SL, Lau J, Smith SJ, Schmid CH, Engelgau MM. Self-management education for adults with type 2 diabetes: a meta-analysis of the effect on glycemic control. Diabetes Care. 2002; 25:1159-71.

116. Norris SL, Engelgau MM, Narayan KM. Effectiveness of self-management training in type 2 diabetes: a systematic review of randomized controlled trials. Diabetes Care. 2001; 24:561-87.

117. Roter DL, Hall JA, Merisca R, Nordstrom B, Cretin D, Svarstad B.

Effectiveness of interventions to improve patient compliance: a meta-analysis. Med Care. 1998; 36:1138-61. 
118. Barlow J, Wright C, Sheasby J, Turner A, Hainsworth J. Self-management approaches for people with chronic conditions: a review. Patient Educ Couns. 2002; 48:177-87.

119. Skinner TC, Cradock S, Arundel F, Graham W. Four theories and a philosophy: self-management education for individuals newly diagnosed with type 2 diabetes. Diabetes Spectrum. 2003; 16:75-80.

120. Pronk NP. Placing Workplace Wellness in Proper Context: Value Beyond Money. Preventing Chronic Disease. 2014; 11:E119.

121. Davis N, Forbes B, Wylie-Rosett J. Nutritional strategies in type 2 diabetes mellitus. Mt Sinai J Med. 2009; 76:257-68.

122. Gill JM, Cooper AR. Physical activity and prevention of type 2 diabetes mellitus. Sports Med. 2008; 38:807-24.

123. Myers J, Prakash M, Froelicher V, Do D, Partington S, Atwood JE. Exercise capacity and mortality among men referred for exercise testing. The New England journal of medicine. 2002; 346:793-801.

124. Kokkinos P, Myers J, Kokkinos JP, Pittaras A, Narayan P, Manolis A, et al. Exercise capacity and mortality in black and white men. Circulation. 2008; 117:614-22.

125. Knowler WC, Barrett-Connor E, Fowler SE, Hamman RF, Lachin JM, Walker EA, et al. Reduction in the incidence of type 2 diabetes with lifestyle intervention or metformin. The New England journal of medicine. 2002; 346:393403. 
126. Nyenwe EA, Jerkins TW, Umpierrez GE, Kitabchi AE. Management of type 2 diabetes: evolving strategies for the treatment of patients with type 2 diabetes. Metabolism. 2011; 60:1-23.

127. AACE. AACE/ACE Comprehensive Diabetes Management Algorithm 2015 and AACE/ACE Diabetes Clinical Practice Guidelines. 2015.

128. Wilding JP. The importance of weight management in type 2 diabetes mellitus. Int J Clin Pract. 2014; 68:682-91.

129. Elder KA, Wolfe BM. Bariatric surgery: a review of procedures and outcomes. Gastroenterology. 2007; 132:2253-71.

130. Kashyap SR, Gatmaitan P, Brethauer S, Schauer P. Bariatric surgery for type 2 diabetes: weighing the impact for obese patients. Cleve Clin J Med. 2010; 77:468-76.

131. Ganguly S, Tan HC, Lee PC, Tham KW. Metabolic bariatric surgery and type 2 diabetes mellitus: an endocrinologist's perspective. J Biomed Res. 2015; 29:105-11.

132. Singh AK, Singh R, Kota SK. Bariatric surgery and diabetes remission: Who would have thought it? Indian J Endocrinol Metab. 2015; 19:563-76. 133. Schmittdiel JA, Brown SD, Neugebauer R, Adams SR, Adams AS, Wiley D, et al. Health-plan and employer-based wellness programs to reduce diabetes risk: The Kaiser Permanente Northern California NEXT-D Study. Prev Chronic Dis. 2013; 10:E15.

134. Gilpatrick DM. Moving clients toward wellness: behavioral change. Clinical nurse specialist CNS. 1989; 3:25-8. 
135. Berry LL, Mirabito AM. Partnering for prevention with workplace health promotion programs. Mayo Clin Proc. 2011; 86:335-7.

136. Cawley J, Price JA. A case study of a workplace wellness program that offers financial incentives for weight loss. Journal of health economics. 2013; 32:794-803.

137. healthcare.gov. Wellness Programs. 2015 Available from: https://www.healthcare.gov/glossary/wellness-programs/.

138. Soeren Mattke HL, John P. Caloyeras, Christina Y. Huang, Kristin R. Van Busum, Dmitry Khodyakov, Victoria Shier. Workplace Wellness Programs Study. In: DOL, editor.: RAND HEALTH; 2013.

139. Schellenberg ES, Dryden DM, Vandermeer B, Ha C, Korownyk C. Lifestyle Interventions for Patients With and at Risk for Type 2 DiabetesA Systematic Review and Meta-analysis. Annals of internal medicine. 2013; 159:543-51.

140. Rossi A. Wellness programs on the rise. Biotechnol Healthc. 2010; 7:2930.

141. Chou HC, Chen WW, Hsiao FY. Acute pancreatitis in patients with type 2 diabetes mellitus treated with dipeptidyl peptidase-4 inhibitors: a populationbased nested case-control study. Drug safety. 2014; 37:521-8.

142. The Diabetes Prevention Program. Design and methods for a clinical trial in the prevention of type 2 diabetes. Diabetes Care. 1999; 22:623-34. 
143. The Diabetes Prevention Program: baseline characteristics of the randomized cohort. The Diabetes Prevention Program Research Group. Diabetes Care. 2000; 23:1619-29.

144. Goetzel RZ, Henke RM, Tabrizi M, Pelletier KR, Loeppke R, Ballard DW, et al. Do workplace health promotion (wellness) programs work? Journal of occupational and environmental medicine / American College of Occupational and Environmental Medicine. 2014; 56:927-34.

145. Roumen C, Blaak EE, Corpeleijn E. Lifestyle intervention for prevention of diabetes: determinants of success for future implementation. Nutr Rev. 2009; 67:132-46.

146. Reduction in the Incidence of Type 2 Diabetes with Lifestyle Intervention or Metformin. New England Journal of Medicine. 2002; 346:393-403.

147. Diabetes Prevention Program Research G. The Diabetes Prevention Program (DPP): description of lifestyle intervention. Diabetes Care. 2002; 25:2165-71.

148. Tuomilehto J, Schwarz P, Lindstrom J. Long-term benefits from lifestyle interventions for type 2 diabetes prevention: time to expand the efforts. Diabetes Care. 2011; 34 Suppl 2:S210-4.

149. Policy DfMSDfPHOoH. Kentucky Diabetes Report. 2015.

150. Swartz A. James Fries: healthy aging pioneer. Am J Public Health. 2008; 98:1163-6. 
151. Taylor RC. An overview of the Hadoop/MapReduce/HBase framework and its current applications in bioinformatics. BMC Bioinformatics. 2010; 11 Suppl 12:S1.

152. Counting Process Style of Input [database on the Internet]. Available from: http://support.sas.com/documentation/cdl/en/statug/63033/HTML/default/viewer.h tm\#statug phreg sect027.htm.

153. Knox KL, Bajorska A, Feng C, Tang W, Wu P, Tu XM. Survival analysis for observational and clustered data: an application for assessing individual and environmental risk factors for suicide. Shanghai Arch Psychiatry. 2013; 25:18394.

154. Koller EA, Chin JS, Conway PH. Diabetes prevention and the role of risk factor reduction in the Medicare population. Am J Prev Med. 2013; 44:S307-16. 155. Bailey RA, Wang Y, Zhu V, Rupnow MF. Chronic kidney disease in US adults with type 2 diabetes: an updated national estimate of prevalence based on Kidney Disease: Improving Global Outcomes (KDIGO) staging. BMC Res Notes. $2014 ; 7: 415$.

156. Prasad H, Ryan DA, Celzo MF, Stapleton D. Metabolic syndrome: definition and therapeutic implications. Postgrad Med. 2012; 124:21-30. 157. Sattar N. Gender aspects in type 2 diabetes mellitus and cardiometabolic risk. Best Pract Res Clin Endocrinol Metab. 2013; 27:501-7.

158. Kautzky-Willer A, Harreiter J, Pacini G. Sex and Gender Differences in Risk, Pathophysiology and Complications of Type 2 Diabetes Mellitus. Endocr Rev. 2016; 37:278-316. 
159. Upadhyay R, Robay A, Fakhro K, Abi Khalil C, Zirie M, Jayyousi A, et al. Role of SLMAP genetic variants in susceptibility of diabetes and diabetic retinopathy in Qatari population. J Transl Med. 2015; 13:61.

160. Menke A, Casagrande S, Geiss L, Cowie CC. Prevalence of and Trends in Diabetes Among Adults in the United States, 1988-2012. JAMA : the journal of the American Medical Association. 2015; 314:1021-9.

161. Spanakis EK, Golden SH. Race/ethnic difference in diabetes and diabetic complications. Curr Diab Rep. 2013; 13:814-23.

162. Boudreau DM, Yu O, Johnson J. Statin use and cancer risk: a comprehensive review. Expert Opin Drug Saf. 2010; 9:603-21.

163. Sattar N, Preiss D, Murray HM, Welsh P, Buckley BM, de Craen AJ, et al. Statins and risk of incident diabetes: a collaborative meta-analysis of randomised statin trials. Lancet. 2010; 375:735-42.

164. Cheung BM, Li C. Diabetes and hypertension: is there a common metabolic pathway? Curr Atheroscler Rep. 2012; 14:160-6.

165. Aronow WS, Fleg JL, Pepine CJ, Artinian NT, Bakris G, Brown AS, et al. ACCF/AHA 2011 expert consensus document on hypertension in the elderly: a report of the American College of Cardiology Foundation Task Force on Clinical Expert Consensus documents developed in collaboration with the American Academy of Neurology, American Geriatrics Society, American Society for Preventive Cardiology, American Society of Hypertension, American Society of Nephrology, Association of Black Cardiologists, and European Society of Hypertension. J Am Coll Cardiol. 2011; 57:2037-114. 
166. Fong DS, Aiello L, Gardner TW, King GL, Blankenship G, Cavallerano JD, et al. Diabetic Retinopathy. Diabetes Care. 2003; 26:s99-s102.

167. Klein R, Klein BE, Moss SE, Wong TY. The relationship of retinopathy in persons without diabetes to the 15-year incidence of diabetes and hypertension: Beaver Dam Eye Study. Trans Am Ophthalmol Soc. 2006; 104:98-107. 168. Yamaoka-Tojo M, Tojo T, Takahira N, Matsunaga A, Aoyama N, Masuda T, et al. Elevated circulating levels of an incretin hormone, glucagon-like peptide1, are associated with metabolic components in high-risk patients with cardiovascular disease. Cardiovasc Diabetol. 2010; 9:17.

169. Huang PL. A comprehensive definition for metabolic syndrome. Dis Model Mech. 2009; 2:231-7.

170. Mayans L. Metabolic Syndrome: Insulin Resistance and Prediabetes. FP Essent. 2015; 435:11-6.

171. Chin JA, Sumpio BE. Diabetes mellitus and peripheral vascular disease: diagnosis and management. Clin Podiatr Med Surg. 2014; 31:11-26. 172. Scott M. Grundy IJB, Gregory L. Burke, Alan Chait, Robert H. Eckel, Barbara V. Howard, William Mitch, Sidney C. Smith and James R. Sowers. Diabetes and Cardiovascular Disease. Circulation. 1999; 100.

173. Davies SW. Clinical presentation and diagnosis of coronary artery disease: stable angina. Br Med Bull. 2001; 59:17-27.

174. Morisaki N, Kawano M, Watanabe S, Saito Y, Yoshida S. Role of obesity in development of ischemic heart disease in elderly diabetic patients. Gerontology. 1992; 38:167-73. 
175. Mottalib A, Sakr M, Shehabeldin M, Hamdy O. Diabetes Remission after Nonsurgical Intensive Lifestyle Intervention in Obese Patients with Type 2 Diabetes. J Diabetes Res. 2015; 2015:468704.

176. Litwak L, Goh SY, Hussein Z, Malek R, Prusty V, Khamseh ME.

Prevalence of diabetes complications in people with type 2 diabetes mellitus and its association with baseline characteristics in the multinational A1chieve study. Diabetol Metab Syndr. 2013; 5:57.

177. Pories WJ. Bariatric surgery: risks and rewards. J Clin Endocrinol Metab. 2008; 93:S89-96.

178. Chang SH, Stoll CR, Song J, Varela JE, Eagon CJ, Colditz GA. The effectiveness and risks of bariatric surgery: an updated systematic review and meta-analysis, 2003-2012. JAMA Surg. 2014; 149:275-87.

179. Keidar A. Bariatric surgery for type 2 diabetes reversal: the risks. Diabetes Care. 2011; 34 Suppl 2:S361-266.

180. Gagnon LE, Karwacki Sheff EJ. Outcomes and complications after bariatric surgery. Am J Nurs. 2012; 112:26-36, quiz 51, 37.

181. Ashkenazy R, Abrahamson MJ. Medicare coverage for patients with diabetes. A national plan with individual consequences. J Gen Intern Med. 2006; 21:386-92.

182. Dormuth CR, Filion KB, Paterson JM, James MT, Teare GF, Raymond $\mathrm{CB}$, et al. Higher potency statins and the risk of new diabetes: multicentre, observational study of administrative databases. BMJ. 2014; 348:g3244. 
183. Ruta LM, Magliano DJ, Lemesurier R, Taylor HR, Zimmet PZ, Shaw JE. Prevalence of diabetic retinopathy in Type 2 diabetes in developing and developed countries. Diabet Med. 2013; 30:387-98.

184. Shah CA. Diabetic retinopathy: A comprehensive review. Indian J Med Sci. 2008; 62:500-19.

185. Tracy JA, Dyck PJ. The spectrum of diabetic neuropathies. Phys Med Rehabil Clin N Am. 2008; 19:1-26, v.

186. Lundbye JB, Thompson PD. Statin use in the metabolic syndrome. Curr Atheroscler Rep. 2005; 7:17-21. 


\section{CURRICULUM VITAE}

NAME: Srikanth Tangelloju

ADDRESS: School of Public Health and Information Sciences 485 Gray Street

Louisville, KY 40202

\section{EDUCATION\&TRAINING}

B.Sc., Agriculture

Acharya N.G. Ranga Agricultural University

Graduated 2003

Post Graduate Diploma in Management (Agri-Business Management)

National Institute of Agricultural Extension Management

Graduated 2005

M.S., Biology

Seton Hall University

Graduated 2009

Ph.D., Public Health Sciences, Health Management \& Systems Science University of Louisville

Anticipated 2016

SAS certified base programmer

\section{PROFESSIONAL EXPERIENCE}

2009 - Present Humana, Inc.

Strategic consultant, Consumer Analytics

Consultant, Consumer Analytics

Consumer analyst, Strategic Analytics

2006 - 2008 Seton Hall University

Graduate research assistant

2005 - 2006 HDFC Bank Ltd

Deputy Manager 